\section{P 001}

\section{Hypotonic shock in mice as in vivo model for} cytotoxic brain oedema formation

T. Fret ${ }^{\star}$, L. Heylen*, M. Nuydens ${ }^{\dagger}$, F. De Jongh ${ }^{\star}$, T. Meert ${ }^{\dagger}$

*ZOL, Department of ICU, Schiepse Bos 6, 3600 Genk;

†anssen Pharmaceutica, Beerse, Belgium

Background and objective: Brain swelling in humans can be subdivided into a combination of vasogenic, cytotoxic or hydrocephalic oedema. Cytotoxic oedema can be determined in animals by injection of solute free water with desmopressin. However, survival rate and time differ among investigators. The aim was standardization and validation of the acute water intoxication animal model.

Methods and materials: Male NMRI mice of $25-40 \mathrm{~g}$ were injected intraperitoneally with $0.2,0.25$ or $0.3 \mathrm{~mL}$ distilled water per $\mathrm{g}$ body weight with $0.4 \mu \mathrm{g} \mathrm{kg}^{-1}$ desmopressin (at room temperature) during standardized laboratory environment conditions. Neurological observation and visual timing to death, determined by last movement, were recorded.
Results: In our laboratory conditions, no mortality after injection of $0.2 \mathrm{~mL} \mathrm{~g}^{-1} \mathrm{BW}$ within the first three observational hours was detected $(n=12)$, however mice were lethargic, suffered ataxia and sometimes experienced muscular fasciculations. Injection of $0.25 \mathrm{~mL} \mathrm{~g}^{-1} \mathrm{BW}$ resulted in a mortality of $42 \%$ (5 of 12 mice) with a survival time of $50,64,72,126$ and 172 min. The remaining mice survived for at least $5 \mathrm{~h}$, but stayed lethargic with few exploratory movements. Injection of $0.3 \mathrm{~mL} \mathrm{~g}^{-1}$ BW resulted in $100 \%$ mortality (42-80 min, median $55 \mathrm{~min}$ ), which was reproducible (three times $n=12$ ) and independent of the initial body weight. The increase in body weight after injection or after death, if higher than $120 \%$, was not correlated with survival time. Survival time of mice under anaesthesia with isoflurane in upright position did not differ from freely moving, awake mice.

Conclusion: Intraperitoneal injection of $0.3 \mathrm{~mL}$ water per gram mouse with desmopressin is a validated and reproducible animal model to mimic exogenous cytotoxic oedema formation. Standardized laboratory conditions and weighing should be part of the protocol. However, technical monitoring of death can improve the exact timing of death.

\title{
NEUROPROTECTION
}

\section{P 002}

\section{An investigation of pulmonary silent aspiration and} pneumonia in ICU, Shiraz, Iran

S. Gholamzadeh, M. Moattari, A. Emad

Shiraz Medical University, Fatemeh Nursing and Midwifery College, Shiraz, Iran

Background and objective: A descriptive investigation was performed to find out the relationship between subclinical aspiration and pneumonia and also to investigate the relationship between gastric $\mathrm{pH}$ and pneumonia in critically ill patients in the acute care setting in Shiraz, Iran.

Methods and materials: The study was done by detecting the existence of glucose in endotracheal secretions and measuring lung $\mathrm{pH}$ values. The subjects were thirty patients with the main criteria of being mechanically ventilated with a Glasgow Coma Score $<8$. The patients were considered to have subclinical aspiration when their endotracheal secretions were positive for glucose (equal to or above $150 \mathrm{mg} \mathrm{dL}^{-1}$ ) and also when they had abnormal lung $\mathrm{pH}$ values.

Results: The result based on the lung $\mathrm{pH}$ value showed that all the patients under study had subclinical aspiration, but on the basis of endotracheal glucose secretion, $90 \%$ of the patient had subclinical aspiration. Based on both lung $\mathrm{pH}$ and the existence of glucose in the endotracheal aspiration, $66.5 \%$ of the patients had subclinical aspiration. The overall rate of pneumonia in the study was $40 \%$. It was concluded that pneumonia had a direct relationship with lung $\mathrm{pH}$ value $(P \leqslant 0.39)$. It also had a direct relationship with the frequency of high endotracheal glucose secretion equal to or above $150 \mathrm{mg} \mathrm{dL}^{-1}(P=0.01)$ and the abnormal lung $\mathrm{pH}$ value $(P \leqslant 0.01)$. There was no relationship between gastric $\mathrm{pH}$ and pneumonia.

Conclusion: Silent aspiration and pneumonia appear to be significant problems in the acute care setting in Shiraz, Iran.

\section{P 003}

The influence of central haemodynamics on cerebral perfusion parameters in patients with intracranial hypertension

A. A. Belkin, V. S. Gromov, A. L. Levit

Clinical Institute of Brain, Yekaterinburg, Russian Federation

Background and objective: The aim of this study was to investigate the relationships between mean arterial pressure (MAP), cardiac output (CO), cardiac index $(\mathrm{Cl})$, systemic vascular resistance (SVR) and cerebral haemodynamic parameters such as blood flow velocity $(\mathrm{Vm})$, pulsative index $(\mathrm{Pi})$ and transient hyperaemic test (THT) in patients with different levels of intracranial hypertension.

Methods and materials: In all, 49 patients from 25 to $65 \mathrm{yr}$ old with different origins of severe cerebral insufficiency, Glasgow Coma Score $<13$ and intracranial hypertension $>15 \mathrm{mmHg}$ as proved by invasive (Codman) measurements were included into this study. Pharmacological tests were undertaken with sympathomimetic drugs: phenylephrine from 0.1 to $0.5 \mu \mathrm{g} \mathrm{kg}^{-1} \mathrm{~min}^{-1}$ and dopamine from 3 to $10 \mu \mathrm{g} \mathrm{kg}^{-1} \mathrm{~min}^{-1}$ for $30 \%$ increase in MAP.

Results: In patients with ICP from 15 to $20 \mathrm{mmHg}$ the mean (SD; range) increase in MAP from $97(75.7 ; 115,29)$ to $131(107.4 ; 135,3)$ was closely connected with $\mathrm{Vm}$ increasing from $63(51.3 ; 113,3)$ to $93(66.5 ; 128,9)(\mathrm{rs}=0.662, P=0.0072)$ and correlated with rise in SVR ( $r s=0.5, P=0.04$ ). In patients with ICP from 21 to $30 \mathrm{mmHg}$ the increase of $\mathrm{Cl}$ from $3.2(2.5 ; 3,89)$ to $3.95(2.9 ; 4,9)$ led to a rise in blood flow velocity $(\mathrm{Vm})$ in the middle cerebral artery from 62.5 (47.8; $98,68)$ to $87.5(55.6 ; 119,65)(\mathrm{rs}=0.507, P=0.0022)$. In patients with ICP more then $30 \mathrm{mmHg}$ we found strong correlation between changes in SVR and $\mathrm{Pi}(\mathrm{rs}=0.629, P=0.028)$. The influence of $\mathrm{Cl}$ on brain perfusion decreased significantly ( $r s=-0.011, P=0.97$ ).

Conclusion: In patients with ICP from 20 to $30 \mathrm{mmHg}$ the preference is given to sympathomimetic drugs increasing cardiac output, for example dopamine. The compensatory role of CPP rising as a part of brain protection is limited and lost when ICP $>30 \mathrm{mmHg}$. 


\section{P 004}

$\mathrm{N}$-terminal pro-brain natriuretic peptide (NT-proBNP) in acute brain diseases: elevated serum levels in patients without sodium imbalance

V. Spatenkova*, A Kazda ${ }^{\dagger}$, P. Skrabalek*, D. Kralova", P. Suchomel ${ }^{\star}$

${ }^{*}$ Hospital, Department of Neurocentre, Liberec; ' Faculty of Medicine, University, Prague; "Masaryk University, Brno, Czech Republic

Background and objective: B-type natriuretic peptide (BNP) is a member of the peptide family which increases diuresis and natriuresis in the kidneys and causes vasodilatation in blood vessels. At present assessment of the fragment $\mathrm{N}$-terminal proB-type Natriuretic Peptide (NT-proBNP) is routinely available. It arises by splitting the precursor during the release of active BNP. Its relationship with hyponatraemia and natriuresis in cerebral salt wasting (CSW) syndrome means that neurointensive care is another possible field for its application. The aim of our study was to evaluate serum NT-proBNP and its relationship with sodium and water metabolism parameters in patients with acute brain diseases without developed CSW syndrome.

Methods and materials: In our retrospective study we observed NT-proBNP values in 18 patients (9 females, 9 males, mean Glagow Coma Score 14 \pm 1 ) with acute brain diseases (subarachnoid haemorrhage 9, intracerebral haemorrhage 2, tumour 5, other 2) with normal renal parameters and New York Heart Association classification I. Serum sodium and osmolality were measured on the day of NT-proBNP sample (day 1) and $24 \mathrm{~h}$ later (day 2). Diuresis, urinary loss of sodium, intake of fluids and sodium, were observed for a period of $24 \mathrm{~h}$ after the NT-proBNP measurement.

Results: Serum levels of NT- proBNP (mean 316.8 \pm $250.3 \mathrm{pg} \mathrm{mL}^{-1}$ ) were statistically significantly higher than normal value (125 $\left.\mathrm{pg} \mathrm{mL}^{-1}, P=0.005\right)$, while no significant change was observed in levels of either serum sodium $(P=0.799)$ or serum osmolality $(P=0.388)$. Furthermore, there was no proven significant correlation between NT-proBNP and the measured parameters.

Conclusion: This study showed a significant elevation of NTproBNP in patients with acute brain diseases which were not accompanied by developed CSW syndrome.

\section{P 005}

Monitoring the haemodynamic effects of carotid endarterectomy with the Pulsatile apparent Resistance, a newly defined blood pressure corrected pulsatility index

A. Schaafsma*, K. W. E. Oudman ${ }^{\dagger}$

*Martini Hospital Groningen, Department of Klinische Neurofysiologie; 'Neuromon BV, Groningen, The Netherlands

Background and objective: Removing a significant stenosis from the internal carotid artery by endarterectomy (CEA) is a major challenge to cerebral autoregulation. In order to better understand and predict these changes we have conducted a prospective study collecting data on ipsilateral and contralateral middle cerebral artery flow velocity (MCAFV), arterial blood pressure (ABP), ECG and end-tidal $\mathrm{CO}_{2}\left(\mathrm{ETCO}_{2}\right)$ prior to $\mathrm{CEA}$ as well as on postoperative days 1 to 3 . From the continuous recording of MCAFV and $\mathrm{ABP}$ we calculated the Pulsatile apparent Resistance $(\mathrm{PaR})$ The $\mathrm{PaR}$ is a new parameter best described as a 'blood pressure corrected pulsatility index' that has been shown to correlate with the haemodynamic consequences of carotid artery stenosis and is calculated from ((diastolic ABP/diastolic MCAFV)-(systolic ABP/systolic MCAFV))/ (mean ABP/mean MCAFV).

Methods and materials: In all, 20 patients underwent a 20-30 min recording a few days before, as well as on days 1,2 and 3 after $\mathrm{CEA} . \mathrm{CO}_{2}$-reactivity testing, both hyperventilation and $\mathrm{CO}_{2}$-retention, was performed preoperatively and on postoperative day 3 . For analysis, patients were subdivided into four groups determined by combinations of ipsilateral vs. contralateral stenosis.

Results: On average there was a postoperative rise in MCAFV simultaneous with a drop in ABP maximal on postoperative day 1 and returning to preoperative levels at postoperative days 2-3 in all groups. These changes however were most marked in patients with the most severe stenosis. In almost all cases the $\mathrm{PaR}$ showed a postoperative increase. A decrease in PaR correlated with high postoperative flow velocities suspect for hyperperfusion syndrome. Such decrease was most likely to occur in patients with extensive cerebrovascular disease who already had a low PaR value preoperatively.

Conclusion: The PaR is a robust parameter that can signal the occurrence of a postoperative hyperperfusion syndrome. A postoperative decrease instead of an increase in $\mathrm{PaR}$ should be taken as a warnng signal.

\section{P 006}

Cerebral vasomotor reactivity (VMR) in migraine with and without aura: a case-control study

Y. Jahangiri Noudeh", M. Rohani ${ }^{\dagger}$, M. Shabani ${ }^{\top}$, F. Sina ${ }^{\dagger}$, A. R. Rezaei Ashtiani ${ }^{\dagger}$

*Iran University of Medical Sciences, Department of Medical Students Research Committee; ' Rasoul-e-Akram Medical Center; "Iran University of Medical Sciences, Faculty of Medicine, Department of Biochemistry, Tehran, Iran

Background and objective: A dysfunction of the autonomic nervous system has long been a subject of considerable debate and a large number of studies have disclosed contradictory results. The aim was to compare cerebral vasomotor reactivity in migraine with aura (MWA) patients with migraine without aura (MWO) ones.

Methods and materials: Ten MWA patients ( 7 females and 3 males; mean age: $39.7 \mathrm{yr}, \mathrm{SD}: 12.0 \mathrm{yr}$ ) and 10 age- and sexmatched ones with MWO $(P=0.303$, Fischer's exact test; $P=1.000, \chi^{2}$ test, respectively) underwent cerebral vasomotor reactivity measurement using transcranial Doppler imaging of the middle cerebral artery (MCA).

Results: A statistically significant decrease in VMR was seen in the migraine with aura group ( $P=0.048$, Fischer's exact test), and systolic, diastolic and mean flow velocities were significantly greater in these patients $(P=0.021, P=0.017$ and $P=0.049$, Fischer's exact test, respectively). There was no significant difference between Gosling PI in the two groups $(P=0.733$, Fischer's exact test). On the other hand, no correlation was found between age and VMR and mean arterial flow velocity (Vmean) values (Pearson correlation $=0.076, P=0.749$ and Pearson correlation $=0.223, P=0.345$, respectively).

Conclusion: An age-independent decrease in cerebral vasomotor reactivity in MWA compared to MWO could support genetic involvement of brain autonomic control pathways in MWA rather than MWO. Nitric oxide (NO) plays a major role, as a second 
messenger, in cerebral autonomic activity. Genetic involvement of its metabolic pathways may be a good explanation for the observed dysfunction in MWA. Further molecular investigations could clarify this question.

\section{P 007}

\section{Cerebral blood flow thresholds for cerebral ischaemia} in head trauma. A systematic review

M. Botteri, E. Bandera, N. Latronico

Spedali Civili, Department of Anaesthesia and Intensive Care, Brescia, Italy

Background and objective: We systematically reviewed the medical literature to evaluate the evidence available, and its methodological adequacy on Cerebral Blood Flow ischaemic thresholds in adults with Post Traumatic Cerebral Ischaemia.

Methods and materials: Electronic database searching using Medline, Embase, and the Cochrane Library, crosschecking of references and contact with experts and authors of primary studies were used. Included in the review were primary studies on adults with Traumatic Brain Injury, with evaluation of CBF thresholds and diagnostic gold standard represented by followup brain CT or brain MR. Two reviewers independently extracted the data and assessed study quality.

Results: In all, 53 out of 253 articles retrieved and reviewed were primary studies on CBF in adult patients with TBI. The only study included in the review was a retrospective cohort study of 14 adult patients with traumatic brain injury which had CBF measurement by PET and in which the diagnosis of PTCl was made by follow-up MRI. The authors reported a CBF threshold differentiating between lesion- non lesion tissue of $15 \mathrm{~mL}$ per $100 \mathrm{~mL} \mathrm{~min}^{-1}$.

Conclusion: There is no evidence available in the medical literature on Cerebral Blood Flow ischaemic thresholds in adults with Post Traumatic Cerebral Ischaemia.

\section{TRAUMATIC BRAIN INJURY}

\section{P 008}

\section{Determination of blood-brain barrier permeability in brain-injured patients by Patlak plot analysis}

\section{Bentzer}

University Hospital Lund, Department of Anestesia and Intensive Care, Lund, Sweden

Background and objective: Increased permeability of the blood-brain barrier (BBB) may represent an important mechanism for oedema formation following traumatic brain injury (TBI). To date no method has been applied for quantitative determination of BBB permeability in this group of patients. The aim was to develop a method for analysis of permeability of the blood-brain barrier following TBI.

Methods and materials: Three patients suffering from TBI were analysed in this pilot study. After CT perfusion scans, patients were scanned every $3 \mathrm{~min}$ for $24 \mathrm{~min}$. The data were exported to an image analysis software and analysed with a specially designed macro in which the transfer constant (Ki), reflecting permeability, for the contrast agent iohexol was determined by Patlak plot analysis. This analysis assumes that the amount of tracer $(\mathrm{Am})$ in a tissue at a given time $(\mathrm{t})$ following injection can be described by the equation, $\mathrm{Am}=\mathrm{Ki} 0 \int \mathrm{tCp}(\mathrm{t}) \mathrm{dt}+\mathrm{ViCpt}$, where $\mathrm{Cp}$ is concentration of tracer in plasma and $\mathrm{Vi}$ is the initial distribution volume of the tracer. By plotting Am/Cpt vs. $0 \int \mathrm{tCp}(\mathrm{t}) \mathrm{dt} / \mathrm{Cpt}$ at multiple time points a linear curve is obtained with the slope of Ki. By calculating $\mathrm{Ki}$ for each voxel, permeability maps of the brain could be generated.

Results: In two of the patients a heterogeneous pattern of permeability could be demonstrated, with permeability being the greatest in the vicinity of focal lesions. Ki was in the range of $0-0.5 \mathrm{~mL} \mathrm{~min}^{-1}$ per $100 \mathrm{~g}$ in tissue appearing normal on CT and reached a maximum of about $5 \mathrm{~mL} \mathrm{~min}^{-1}$ per $100 \mathrm{~g}$ around focal lesions. In one patient, no focal changes could be detected on CT or on the permeability map.

Conclusion: Patlak plot analysis in brain-injured patients yield detailed quantitative data on permeability which are in the range of those reported for stroke patients and may further our understanding of the mechanisms of oedema formation.

\section{P 009}

Traumatic brain injury (TBl): clinical and epidemiological analysis in a Spanish ICU J. Homar ${ }^{*}$, A. Rossello ${ }^{\dagger}$, J. Perez ${ }^{\dagger}$, J. A. Llompart ${ }^{\dagger}$, J. M. Abadal ${ }^{\dagger}$, G. Frontera ${ }^{\dagger}$

*Hospital Son Dureta, Department of Intensive Care Unit, Palma; ${ }^{\dagger}$ Hospital Universitario Son Dureta, Palma De Mallorca, Spain

Background and objective: We aimed to describe the epidemiological and clinical characteristics of TBI patients admitted to our ICU in a teaching hospital with neurosurgical services.

Methods and materials: This was a prospective study that analysed all TBI patients admitted to our ICU unit from January 2000 to December 2006. Clinical and epidemiological data was collected on all patients: gender, age, cause of the injury, Glasgow Coma Score (GCS), isolated TBI or multiple injuries, Injury Severity Score (ISS), radiological classification according to Traumatic Coma Data Bank (TCDB), intracranial pressure (ICP) monitoring, intracranial hypertension $(\mathrm{ICH})$ and mortality. Revised trauma score (RTS) was recorded pre-ICU admission. Data was analysed using non-parametric tests. Results: During the 5-yr period 480 patients were studied. They had a mean age of $41.4 \mathrm{yr}(\mathrm{Cl} 95 \%$ : 39.6-43.2) and $82.3 \%$ were males. The main cause of TBI was road traffic accidents in 285 patients (59.4\%), mainly motorbike and isolated TBI in 117 patients $(24.3 \%)$. The most frequent radiological finding was diffuse injury II (TCDB) in 255 patients (53.1\%). The mean RTS and ISS of the whole sample were 9.5 and 20.3, respectively. Correlation of severity of $\mathrm{TBI}$ and $\mathrm{ICP}, \mathrm{ICH}$ and mortality is shown in Table 1. Severe TBI mortality was significatively related to older age, higher ISS and lower GCS values (Table 2).

Table 1. Severity of TBI and ICP, ICH and mortality.

\begin{tabular}{lrcc}
\hline & ICP & ICH & ICU mortality* \\
\hline Mild $(n=94)$ & 11 & 4 & $1(1.06 \%)$ \\
Moderate $(n=121)$ & 35 & 21 & $7(5.8 \%)$ \\
Severe & 147 & 73 & $56(21.13 \%)$ \\
\hline
\end{tabular}

Mild TBI: GCS between 13-15; Moderate TBI: GCS between 9-12; Severe TBI: GCS between 3-8.

ICP: patients with ICP catheter: ICH: patients with intracranial hypertension: *mortality due to TBI. 
Table 2. Correlation between severe $\mathrm{TBI}$ mortality and age, GCS and ISS.

\begin{tabular}{lccc}
\hline & Survival & Death & $P$ value \\
\hline Age & 36.7 & 47.2 & $<0.01$ \\
GCS & 6.11 & 4.68 & $<0.01$ \\
ISS & 20.64 & 29.18 & $<0.01$ \\
\hline
\end{tabular}

Conclusion: TBI patients admitted in our ICU have clinical and epidemiological characteristics as well as mortality rates similar to other ICU units that classically have been already published.

\section{P 010}

\section{Prospective outcome after severe head injury, in patients treated with an ICP targeted therapy}

M. Olivecrona, M. Rodling-Wahlström, S. Naredi, L-O. D. Koskinen

University Hospital, Department of Neurosurgery, Ume, Sweden

Background and objective: Since 1994 we have used a protocol-guided treatment for severe traumatic brain injury (sTBI) based on the Lund concept. Between Jan 2002 and Dec 2005 we conducted a prospective study of sTBI treated in a protocol guided way. The aim was to prospectively study the outcome after STBI treated with an ICP targeted therapy based on the Lund concept.

Methods and materials: Patients with STBI admitted between 2002 and 2005 were eligible. Inclusion criteria: GCS at intubation and sedation $\leqslant 8$, age $15-70 \mathrm{yr}$, first recorded CPP $\geqslant 10 \mathrm{mmHg}$, and arrival within $24 \mathrm{~h}$. Exclusion criteria: pregnant or lactating women and penetrating injury. All patients received an intraparenchymal ICP measuring device on arrival. They were treated in a protocol guided manner based on the Lund concept. The GOS were evaluated by independent staff at 3 and 6 months after injury.

Results: In all, 48 patients (18 females, 30 males) mean age $35 \mathrm{yr}$ were included. No patient was lost to follow-up. Median GCS at intubation and sedation was $6(3-8)$, mean \pm SD $5.3 \pm 0.2$. Many were multi-trauma with median (range) ISS 29 (9-50). The APACHE score was $20.8 \pm 0.7(12-32)$. Two patients $(4.2 \%)$ died during cerebral intensive care. One of these died directly due to high ICP. The median GOS at 3 months was 4, mortality $12.5 \%$ and the favourable outcome (GOS 4-5) $52 \%$ (60\% in survivors). At 6 months the median GOS was 4, total mortality $14.6 \%$ and the favourable outcome $54 \%$ (63\% in survivors). Excluding subjects with dilated fixed pupils and GCS 3 at inclusion the mortality at 3 and 6 months was $5 \%$ and $8 \%$, respectively and favourable outcome $57 \%$ and $59 \%$, respectively.

Conclusion: Treatment with an ICP targeted therapy seems to give favourable results and low mortality. Our prospective findings are in the range of our earlier reported retrospective data.

\section{P 011}

\section{Relationship between brain tissue oxygen tension and CT perfusion: preliminary results}

J. Pérez-Bárcena, M. Picado, J. Ibañez,

J. A. Llompart-Pou, A. Mas, M. Brell, J. H. Homar,

A. Moll, J. M. Abadal

Hospital Universitario Son Dureta, Department of Intensive Care Unit, Palma De Mallorca, Spain
Background and objective: Current intraparenchymal monitors provide focal measurements of brain tissue oxygen tension $\left(\mathrm{PbrO}_{2}\right)$ and their target is the white matter of one hemisphere. CT perfusion (CTP) imaging is an emerging technique that is increasingly used to evaluate patients with acute ischaemic stroke. By injecting contrast material and by performing sequential imaging of the section of interest, the mean transit time (MTT) through brain tissue, the cerebral blood flow (CBF) and the cerebral blood volume (CBV) can be evaluated for a predetermined region of interest (ROI). However the use of CTP has not been thoroughly evaluated in traumatic brain injured (TBI) patients, where tissue hypoxia is due to multiple causes. The aim was to determine if there is a relationship between CTP imaging parameters and $\mathrm{PbrO}_{2}$ values.

Methods and materials: Nine patients underwent continous $\mathrm{PbrO}_{2}$ monitoring with catheters placed into the white matter of the frontal lobes. The ROI selected was surrounding the oxygen probe. CTP analysis software was used to measure the MTT, $\mathrm{CBF}$ and $\mathrm{CBV}$ in the ROI described previously. For correlation, $\mathrm{PbrO}_{2}$ levels and other physiologic parameters were recorded at the time of CTP.

Results: $\mathrm{PbrO}_{2}$ minimum and maximum values at the time of CTP were: $11-113 \mathrm{mmHg}$; MTT 2.3-10 s; CBV $1.1-7.7 \mathrm{~mL}$ per $100 \mathrm{~g}$ and $\mathrm{CBF} 13.9-98.5 \mathrm{~mL}$ per $100 \mathrm{~g} \mathrm{~m}^{-1}$. $\mathrm{PbrO}_{2}$ correlated with CBF $(r=0.83 ; P=0.05)$. MTT or CBV were not correlated with $\mathrm{PbrO}_{2}$. $\mathrm{PbrO}_{2}$ also correlated with the $\mathrm{CBF}$ of the ROI located at the anterior cerebral artery region $(r=0.73 ; P=0.02)$ but not with the middle and posterior cerebral arteries.

Conclusion: CTP assessment of ROI surrounding an oxygen probe in the parenchymal brain tissue is feasible and showed a significant correlation between $\mathrm{PbrO}_{2}$ and $\mathrm{CBF}$.

\section{P 012}

\section{Correlation of cytokine levels in peripheral blood, brain interstitial space and cerebrospinal fluid in traumatic head injury}

J. Pérez-Bárcena*, J. Ibañez*, J. A. Llompart-Pou*, M. Brell*, P. Llinas*, J. H. Homar*, J. M. Arteaga*, J. M. Abadal ${ }^{\star}$, J. M. Raurich ${ }^{\star}$, J. Pierola ${ }^{\dagger}$, C. Crespi ${ }^{\dagger}$, V. Regueiro", J. A. Bengoechea"

*Hospital Universitario Son Dureta, Department of Intensive Care Unit; 'Investigation Unit, Hospital Son Dureta; "Fundación Caubet-Cimera, Palma De Mallorca, Spain

Background and objective: The inflammatory response in traumatic brain injury (TBI) patients has been classically studied through samples obtained from peripheral blood (PB) and cerebrospinal fluid (CSF). Recently microdialysis monitoring with a membrane cut-off of $100 \mathrm{Kda}$ has allowed us to recover samples directly from the brain parenchyma, but it is not clear if there is a correlation between the cytokine levels measured through microdialysis, PB and CSF. The aim was to study if there is a correlation in the cytokine levels measured through microdialysis, PB and CSF.

Methods and materials: In all, 21 paired samples obtained from microdialysis, PB and CSF were simultaneously collected every $8 \mathrm{~h}$ in a TBI patient. All the 63 samples were later analysed in our laboratory for IL12, TNF, IL10, IL6, IL1 $\beta$ and IL8.

Results: The results are expressed as the mean value and standard deviation (Fig. 1). The only values that present a statistically significant correlation are: IL8 measured in PB with IL8 in microdialysis (Pearson correlation $=0.56 ; P=0.008$ ); and TNF in PB with TNF measured in microdialysis (Pearson correlation $=0.49 ; P=0.02$ ). 


\begin{tabular}{llllllc}
\hline & IL12 $(\mathrm{pg} / \mathrm{ml})$ & TNF $(\mathrm{pg} / \mathrm{ml})$ & IL10 $(\mathrm{pg} / \mathrm{ml})$ & IL6 $(\mathrm{pg} / \mathrm{ml})$ & IL1 $\beta(\mathrm{pg} / \mathrm{ml})$ & IL8 $(\mathrm{pg} / \mathrm{ml})$ \\
\hline Peripheral blood & $3.2 \pm 9.3$ & $2.3 \pm 6.5$ & $2.8 \pm 4.3$ & $172.1 \pm 296.8$ & $3.2 \pm 1.5$ & $25.3 \pm 11.9$ \\
Microdialysis & $1.7 \pm 1.2$ & $1.6 \pm 1.5$ & $0.3 \pm 0.6$ & $635.8 \pm 460.3$ & $5.0 \pm 2.0$ & $547.1 \pm 373.8$ \\
Cerebrospinal fluid & $1.9 \pm 1.5$ & $1.7 \pm 1.3$ & $1.2 \pm 1.0$ & $2620.6 \pm 3068.8$ & $4.8 \pm 2.3$ & $493.5 \pm 364.4$ \\
\hline
\end{tabular}

Conclusions: There is a poor correlation among the levels of cytokines measured in the PB, CSF and microdialysis.

Acknowledgement: This study was funded by a grant from the Fondo de Investigación Sanitaria FIS 070307.

\section{P 013}

\section{Abnormal microcirculation in traumatic brain-injured patients during surgery of cerebral contusions}

J. Pérez-Bárcena, J. Ibañez, J. A. Llompart-Pou, M. Brell, P. Llinas, J. H. Homar, J. M. Abadal

Hospital Universitario Son Dureta, Department of Intensive Care Unit, Palma De Mallorca, Spain

Background and objective: The detection and treatment of cerebral ischaemia constitutes one of the main objectives in traumatic brain injured (TBI) patients. Multimodal monitoring has shown us that tissue hypoxia has multiple causes. The alteration of the microcirculation could be one of these causes but its study has several technical limitations. Recently the Sidestream Dark Field (SDF) (MicroScan ${ }^{\mathbb{R}}$ Microvision-Medical; The Netherlands) has been commercialized. The aim was to visualize and quantify the cerebral microcirculation in TBI patients with brain contusions who required a surgical evacuation with the SDF system. The images of the control group were recorded on a macroscopically healthy brain of neurosurgical patients without TBI that required a programmed surgical procedure.

Methods and materials: The brain microcirculation was measured using the SDF. This technique eliminates directly reflected green polarized light from an organ surface using an orthogonally placed analyser. Later we used the microvascular analysis software to determine functional density of small $(<20 \mu \mathrm{m})$, medium $(20-40 \mu \mathrm{m})$ and large $(>40 \mu \mathrm{m})$ microvessels.

Results: We present the preliminary results from a control (Fig. 1) and a TBI case (Fig. 2). The total length of the vessels was $5120 \mu \mathrm{m}$ in the control and $625 \mu \mathrm{m}$ in the TBI patient. In the controls, vessels occupied $14.2 \%$ of the total area studied and in the TBI patient just $2.5 \%$. In the table we show the distribution according to the size of the vessels.

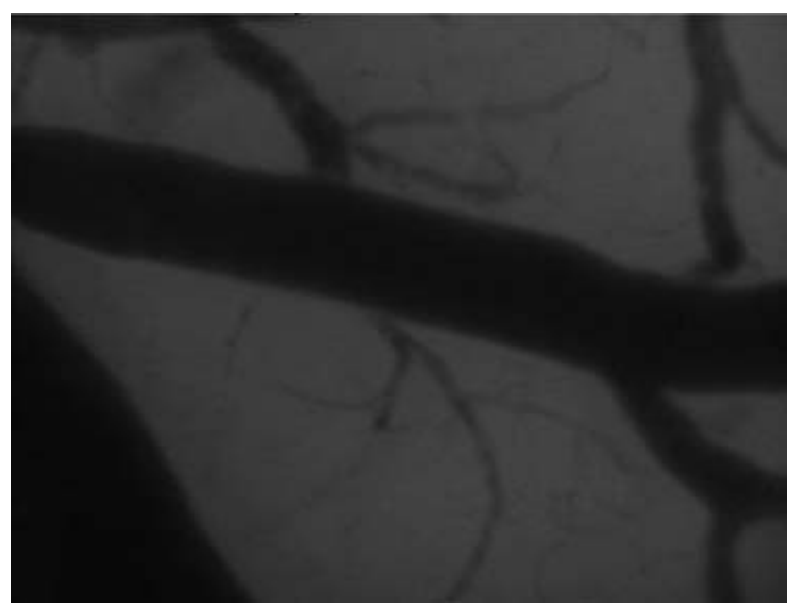

Figure 1

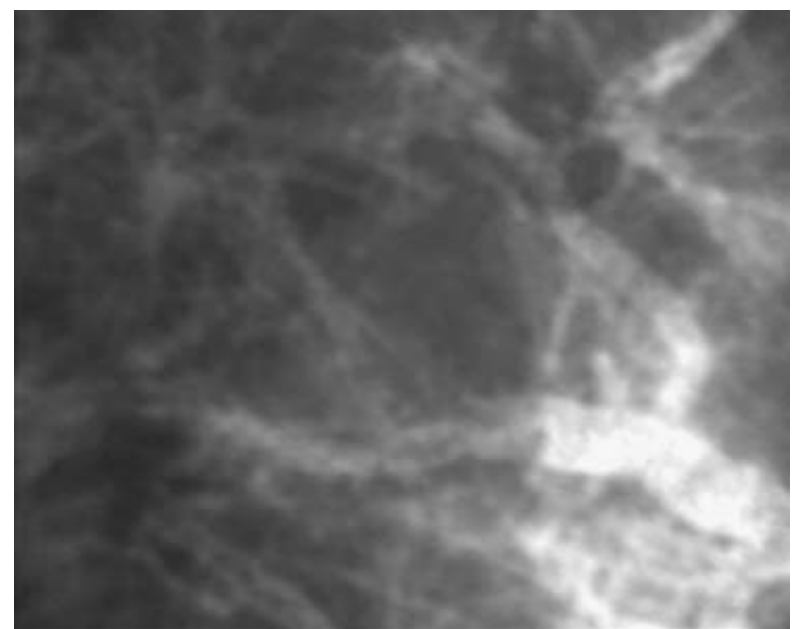

Figure 2

Table 1

\begin{tabular}{ll}
\hline $\begin{array}{l}\text { Length } \\
\text { Control/case }\end{array}$ & \\
$0-20 \mathrm{~m}$ & $77.87 \% / 55.14 \%$ \\
$20-40 \mathrm{~m}$ & $12.31 \% / 41.14 \%$ \\
$40-60 \mathrm{~m}$ & $6.66 \% / 3.54 \%$ \\
$60-80 \mathrm{~m}$ & $2.71 \% / 0 \%$ \\
$80-100 \mathrm{~m}$ & $0.4 \% / 0 \%$ \\
& \\
Area & \\
Control/case & \\
$0-20 \mathrm{~m}$ & \\
$20-40 \mathrm{~m}$ & $6.87 \% / 0.73 \%$ \\
$40-60 \mathrm{~m}$ & $3.11 \% / 0.84 \%$ \\
$60-80 \mathrm{~m}$ & $1.36 \% / 0.1 \%$ \\
$80-100 \mathrm{~m}$ & $0.29 \% / 0 \%$ \\
& $0.29 \% / 0 \%$ \\
\hline
\end{tabular}

Conclusion: SDF imaging allows direct in vivo observation of the cerebral microcirculation enabling us to visually observe and quantify it.

\section{P 014}

\section{Colloid vs. crystalloid fluid volume resuscitation in experimental traumatic brain injury with haemorrhagic shock}

M. B. Jungner ${ }^{\star}$, P-O. Grände ${ }^{\dagger}$, P. Bentzer ${ }^{\dagger}$

*Lund University Hospital, Department of Anaesthesiology and Intensive Care; ' Lund University, Department of Anaesthesiology and Intensive Care, Lund, Sweden

Background and objective: The goal of fluid resuscitation in traumatic brain injury (TBI) with hypovolaemic shock is to restore normovolaemia and increase cerebral blood to avoid secondary brain injuries. Theoretically, colloids may be preferable to 
crystalloids, as they maintain plasma colloid osmotic pressure and thus potentially counteract vasogenic brain oedema. Previous studies have reported conflicting results and the effect of resuscitation on blood volume has not been investigated. The aim was to determine the impact on cortical water content of resuscitation to equal blood volume with crystalloids or colloids following TBI with haemorrhagic shock.

Methods and materials: Male rats were subjected to fluid percussion injury and bled a total $20 \mathrm{~mL} \mathrm{~kg}^{-1}$ bodyweight, approximately $1 / 3$ of total blood volume. After randomization, rapid resuscitation was performed with either $20 \mathrm{~mL} \mathrm{~kg}^{-1}$ isotonic albumin (group A20) or 50 and $90 \mathrm{~mL} \mathrm{~kg}^{-1}$ of isotonic Ringer's acetate, respectively (groups C50 and C90). After $3 \mathrm{~h}$, animals were decapitated and cortical water content was determined using a wet/dry weight method. Plasma volume was determined after brain trauma and at the end of the experiment using a 125-I-albumin tracer technique. One-Way ANOVA followed by Student-Neuman-Keuls test was used for statistical analysis. Data are presented as mean \pm SD.

Results: Blood volumes were equal in A20 $(n=16)$ and C90 $(n=16)$, with a reduction by $10 \pm 9 \%$ from baseline values, and lower in C50 (19 $\pm 10 \%$ reduction from baseline, $n=9)$. Colloid osmotic pressure was significantly lowered in C50 and C90 $(-34 \pm 8 \%$ and $-38 \pm 7 \%)$, while it remained at baseline level in A20, $-4 \pm 10 \%(P<0.001)$. Osmolality and all other haemodynamic data were equal. Cortical water content in A20 was significantly lower than in C90, $(81.36 \pm 0.58 \%$ and $82.02 \pm$ $0.74 \%$, respectively, $P<0.04$ ) and did not differ from C50 $(81.82 \pm 0.83 \%)$

Conclusion: Following experimental TBI with haemorrhagic shock, crystalloids promote more brain oedema than albumin if administered to reach equal intravascular volume expansion.

\section{P 015}

\section{Our experiences with bilateral multimodal monitoring in brain injury}

J. Prochazka, A. Hejcl, M. Bolcha, M. Sames

Masaryk Hospital, Central ICU, Usti Nad Labem, Czech Republic

Background and objective: The therapy of patients with severe traumatic brain injury (TBI) is still insufficient which often results in poor outcome. This is partly due to limited information that we can receive about the state of the brain using standard monitoring methods. In this study we compared results of multimodal monitoring in both brain hemispheres.

Methods and materials: Since January to July 2007 we performed bilateral multimodal monitoring using bolt technique in 9 patients with severe brain injury. We measured intracranial pressure, brain-tissue oxygen (Licox) and brain metabolism using microdialysis in the injured hemisphere and in the healthy hemisphere as a reference.

Results: Monitored periods were between $4 \mathrm{~h}$ and 2 weeks. In most patients we performed perfusion brain CT to confirm correct probe position. Five patients underwent decompressive craniectomy before or during monitoring. In four patients there were no significant differences between both hemispheres; in one patient we observed physiological results of all measured parameters, in four patients we saw differences between both hemispheres with worse values in injured one. We did not observe any complications. Good clinical outcome we observed in two patients (one with physiological values and one in group without differences), moderate outcome was in five patients (two in group without differences, three in group with worse values in injured hemisphere), two patients died (one patient in each group).
Conclusion: Our data show the importance of correct probes placement. Probe placement in injured hemisphere may show early signs of deterioration when compared with monitoring the healthy hemisphere. Clinical outcome correlated well with measured data. This approach may also be useful as a prediction of the fate of brain injury.

\section{P 016}

\section{ICP monitorization in severe head injury}

I. Elmaci, C. Melek, B. Sen, F. Bölükbasi, T. Kasikci

Göztepe Education and Research Hospital, Department of Neurosurgery, Göztepe Education and Research Hospital, Istanbul, Turkey

Background and objective: Monitoring of severely head-injured patients is essential to optimize cerebral haemodynamics and thus to minimize secondary injuries. Head-injured patients may require specific ICP treatment to maintain it below $20-25 \mathrm{mmHg}$ and to obtain an adequate cerebral perfusion pressure (CPP) greater than $60-70 \mathrm{mmHg}$. We investigated the correlation between cerebral variables obtained in the first 7 days and survival.

Methods and materials: This is a prospective study in nine patients in a six-bed adult ICU. Nine patients with severe head trauma $(\mathrm{GCS}<8$ ) were included. In our study we assessed ICP, CPP, CVP, MAP, $\mathrm{PaCO}_{2}$, TCD (Vmax, Pi) parameters, length of stay (LOS) and outcome. The intracranial hypertension treatment protocol proceeded according to the ICP values.

Results: Nine patients ( 8 male/ 1 female) ages between 18 and 63 had mean GCS of 6 . They were sedated and ventilated mechanically. ICP was measured through an intraventricular catheter. The mean duration of ICP catheter was 7 days, mean average ICP was $18( \pm 2) \mathrm{mmHg}$, mean average CPP was 72 $( \pm 2) \mathrm{mmHg}$, cerebrospinal fluid was withdrawn in 4 patients. Low doses of mannitol were infused in all patients. Three patients required agressive treatment (vasopressors and barbiturate infusions). The admission GCS was worse for patiens with worse outcome.

Conclusion: The management of severe head injury should include intubation, followed by ICP monitoring. Immediate reduction of raised ICP to less than $20 \mathrm{mmHg}$ and maintenance of CPP at levels higher than $70 \mathrm{mmHg}$ seems to be mandatory in severe head injury treatment protocols.

\section{P 017}

\section{Anaesthesia care for head trauma - a Lithuanian survey}

\section{Bilskiene, A. Macas, A. Karbonskiene}

Kaunas University of Medicine, Department of Anaesthesiology, Kaunas, Lithuania

Background and objective: Anaesthesia management of patients suffering head trauma still remains challenging. Different aspects of care such as choice of methods of anaesthesia and selection of place for postoperative management of these patients are widely discussed. The aim was to present the current situation of anaesthesia for head trauma in tertiary care centres in Lithuania.

Methods and materials: Retrospective study. A standard questionnaire was sent to the heads of anaesthesia departments of neurosurgical referral hospitals.

Results: All five neurosurgical referral hospitals responded to the questionnaire. Responses presented data of 1555 patients (965 $(62.1 \%)$ males, 590 (37.9\%) females) who underwent anaesthesia for head trauma in 2006. The average age of the patients was $37.6 \pm 18.4 \mathrm{yr} ; 9.1 \%$ patients were ASA class V; $27.5 \%$ - IV; 
$39.4 \%$ - III; $13.8 \%$ - II; and 10.2\% - I. In all, 904 (58.1\%) patients were operated on for subdural haematomas, 234 (15\%) for epidural haematomas, $326(21 \%)$ for chronic subdural haematomas and 91 (5.9\%) for scull impression fractures. In all, 1474 (94.8\%) patients received general anaesthesia with controlled ventilation and $81(5.2 \%)$ received intravenous sedation and monitoring. Sedation and monitoring was used exceptionally for patients with chronic subdural haematomas. The average duration of anaesthesia was $162.1 \pm 59.1 \mathrm{~min}$., while duration of surgery was $147.3 \pm 51.2 \mathrm{~min}$. In all, $174(11.2 \%)$ patients were transferred to a regular ward after the surgery, 106 (6.8\%) went to the postoperative recovery room and $1275(82 \%)$ to the ICU. In all, $263(17.8 \%)$ patients managed with controlled ventilation were extubated in the operating theatre while the remainder were transferred to the ICU for prolonged ventilation. Hyperventilation and cooling were predominant neuroprotection methods.

Conclusion: The admission policy of patients suffering head trauma is not yet standardized in Lithuania. General anaesthesia with controlled ventilation during surgery and prolonged ventilation in the postoperative period are essential elements of anaesthesia care.

\section{P 018}

Ten years experiences with the lund concept and early rehabilitation in patients whith severe traumatic brain injury

W. Scherer, D. Uekötter, B. Hoffmann, A. Sephernia

Clemenshospital, Department of Anaesthesiology and Intensive Care, Muenster, Germany

Background and objective: The Lund Concept is a therapeutic concept for brain-injured patients aiming for the reduction of increased intracranial pressure (ICP) by limiting transcapillary fluid filtration through an opened blood-brain barrier. The aim of the study was to verify the favourable results of brain-injured patients, published by the Lund group.

Methods and materials: A group of 88 patients is presented, mean age $41.8 \mathrm{yr}(14-81 \mathrm{yr})$, initial GCS $<8$, epidural haematoma (9), subdural haematoma (35), intracerebral haematoma (13) and other non-space-occupying lesions (29). Following ICP/CPP monitoring and evacuation of mass lesions, patients were sedated, ventilated and treated according to the Lund concept aiming at euvolaemia using colloids, normothermia, normoglycaemia and normotension. Patients with stable cardio-respiratory function were transferred within the hospital to an early rehabilitation unit with ICU

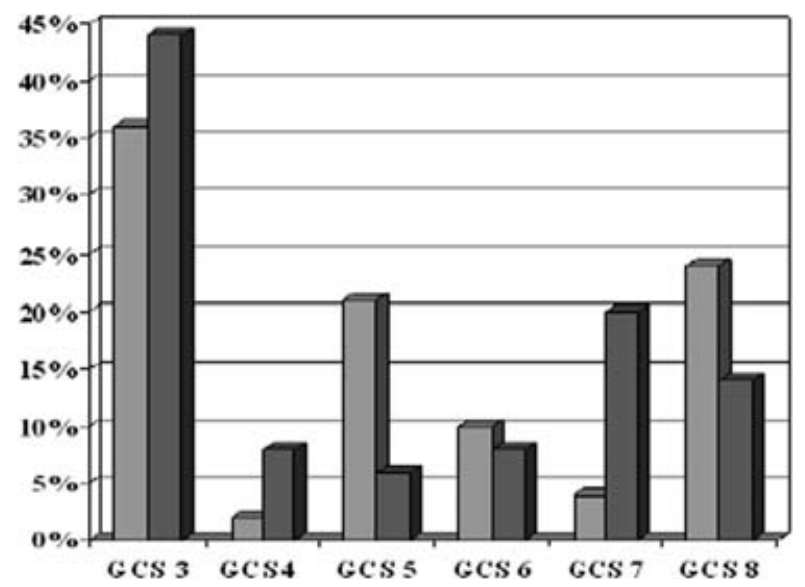

Figure 1.

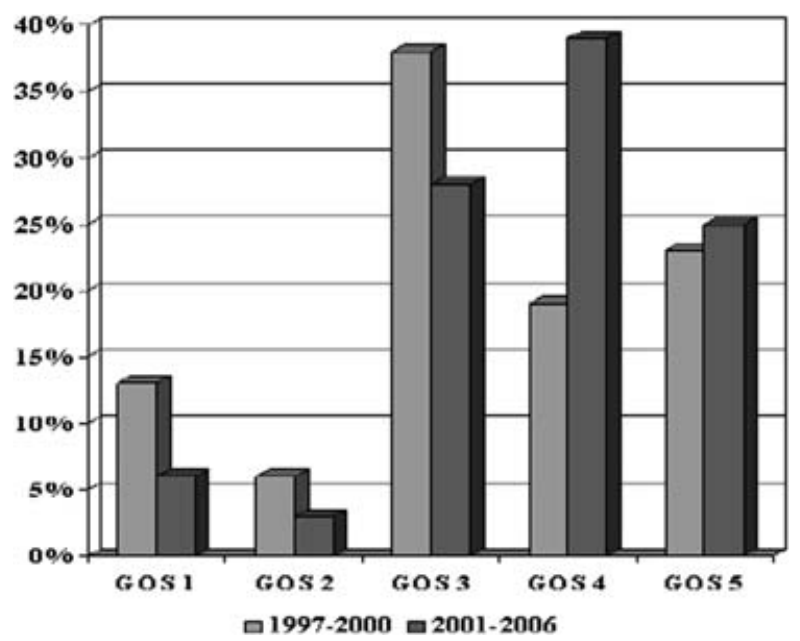

Figure 2.

monitoring. The rehabilitation programme consisted of interventions in neuropsychology, physiotherapy, speech therapy, music therapy and occupational therapy.

Results: The results of two different periods of analysis (1997-2000 and 2001-2006) are shown in Figures 1 and 2. Figure 1 shows initial GCS Scores with an increasing number of patients with GSC 3 and GCS 7 in the second period of analysis (2001-2006). Figure 2 shows the GOS scores $1 \mathrm{yr}$ after injury demostrating a reduction in mortality (GOS 1) and a decreased number of patients with GOS 3 and an increased number of patients with GOS 4 in the second period of analysis.

Conclusion: This study shows that implementing the Lund Concept in a teaching hospital in Germany was able to reproduce the same low mortality rate and high rate of favourable outcome in severely head-injured patients. These results compare well with recent results published by the Lund group.

\section{References}

1. Eker C et al. Brain Injury 2000; 14: 605.

2. Naredi S et al. Acta Anesthesiol Scand 2001; 45: 402.

\section{P 019}

\section{Prognostic value of specific neural enolase in head trauma}

M. Souissi, W. Trabelsi, M. Soussi, M. Achour, K. Baccar,

C. Kaddour

National Institute of Neurology, Department of Anesthesia and intensive Care, La Rabta, Tunisia

Background and objective: Searching for a correlation between plasma level of specific neural Enolase (SNE) and the prognosis of head trauma in the short term.

Methods and materials: An open prospective study over 15 months including 35 patients with head trauma. Criteria of non inclusion were age $<15 \mathrm{yr}$ and head trauma requiring neurosurgery. Clinical basic neurologic survey and CT scan (Fischer and Marshall classification) were repeatedly carried out. A cerebral CT scan was systematically performed $48 \mathrm{~h}$ after hospitalization or in case of deterioration. Plasma level of SNE was measured on the first, third and fifth day of hospitalization, using an ELISA technique with an immunoenzymatic method (kit CanAg Diagnostics) in a Dias Orin automated analyser (Eti_max 3000). Statistical analysis used $\chi^{2}$ test and variance analysis. Values were expressed as mean \pm SD. 
Results: Mean age $39 \pm 17 \mathrm{yr}$; sex ratio $=4 ; 80 \%$ of patients had severe head trauma. Mortality: 16 patients (45.7\%), mean level of SNE was $7.02+3.86 \mu \mathrm{g} \mathrm{mL}^{-1}$ with a statistically significant difference $(P=0.001)$ (pathologic value above $\left.12.5 \mu \mathrm{g} \mathrm{mL}^{-1}\right)$. In all, 17 patients $(48.56 \%)$ had a pathologic SNE plasma level in the first day. All patients $(n=9)$ whose SNE plasma level had still been elevated until the third or the fifth day, died. Plasma level was higher in patients who died in comparison to that of surviving patients; $25.74+22.39 \mu \mathrm{g} \mathrm{mL}^{-1}$ vs. $11.51+4.56 \mu \mathrm{g} \mathrm{mL}^{-1}(P=0.032)$. All nonsurvivors had a higher plasma level of SNE in the fifth day, 6 of them had a normal value in the first day. Patients with GCS $<8$ had a first day plasma level so far higher, $24.89+25.27 \mu \mathrm{g} \mathrm{mL}^{-1}$ vs. $15.25+11.6 \mu \mathrm{g} \mathrm{mL}^{-1}$; SNE plasma level was not correlated with the severity of the subarachnoÿd haemorrhage.

Conclusion: The persistence of a high plasma level of specific neural enolase or its elevation represents a short term prognostic factor.

\section{P 020}

Decompressive craniectomy and duraplasty for refractory intracranial hypertension in head injury

M. Souissi, W. Trabelsi, Z. Zaghdoudi, M. Soussi,

N. Baffoun, C. Kaddour

National Institute of Neurology, Department of Anesthesia and Intensive Care, La Rabta, Tunisia

Background and objective: Severe traumatic brain injury (TBI) is associated with a high mortality and morbidity. Decompressive craniectomy has regained therapeutic interest during past years, however, treatment guidelines consider it a last resort treatment strategy for use only after failure of conservative therapy. In recent years there has been a renewed interest with head injuries and severe intracranial hypertension, as well as in patients with no surgical mass lesion.

Methods and materials: We report on the clinical course of five patients with decompressive craniectomy after TBI with no surgical mass lesion in our multidisciplinary ICU. The standard protocol of intensive care treatment included sedation and muscle relaxation, normothermia, mild hyperventilation and catecholamines to maintain an adequate cerebral perfusion pressure. Decompressive craniotomy including dura opening was initiated according to the following criteria: the existence of clinical signs of cerebral herniation, correlated with abnormalities in computed tomography scan including diffuse or unilateral brain swelling and/or cerebral herniation despite maximally intensified conservative therapy (optimized sedation and ventilation, barbiturates or mannitol).

Results: In all cases the follow-up computed tomography scan demonstrated a resolution of cerebral herniation. At discharge, two patients were without disability, two patients had a mild arm hemiparesis. A spastic hemiplegia was observed in one patient.

Conclusion: These observations indicate feasibility and efficacy of decompressive craniectomy in malignant intracranial pressure rise secondary to TBI. Further controlled trials are necessary to evaluate the indication and standardization of decompressive craniectomy as a second tier standard therapy in severe head injury.

\section{P 021}

\section{Thrombocytosis after traumatic brain injury: incidence, aetiology and outcome}

M. Souissi, W. Trabelsi, M. Soussi, Z. Zaghdoudi,

M. Achour, K. Baccar, C. Kaddour

National Institute of Neurology, Department of Anesthesia and Intensive Care, La Rabta, Tunisia
Background and objective: Trombocytosis refers to a platelet count above the normal value.There is little literature regarding thrombocytosis after traumatic brain injury (TBI). The purpose of this study was to determine the incidence, aetiology and outcome of thrombocytosis after TBI.

Methods and materials: We retrospectively reviewed the records of all patients with TBI, admitted to our ICU between January 2003 and December 2005. Data related to age, sex, infections in the ICU, thromboembolic events, duration of ICU stay, severity scores, and outcome were recorded. We defined thrombocytosis as a platelet count of greater than $500 \times 10^{9} \mathrm{~L}^{-1}$. Statistical analysis (SPSS v11.5) was based on the $\chi^{2}$ test and $t$-test corrected by the Fisher exact test. A value of $P<0.05$ was considered significant.

Results: In all, 169 patients with TBE were studied. The mean age was $31 \mathrm{yr}$ (range 7-78), sex ratio 2.8. Patients were divided into two groups, those with thrombocytosis $(\mathrm{TH}+)$ and those without thrombocytosis ( $\mathrm{TH}-$ ). Thrombocytosis was observed in 28 patients $(16.7 \%)$. The main causes of thrombocytosis were pneumonia $(18,64 \%)$, urinary tract infections $(12,43 \%)$, systemic inflammatory syndrome $(10,36 \%)$ and respiratory distress syndrome $(7,25 \%)$. Thromboembolic events occurred in 3 patients. Median length of stay of $\mathrm{TH}+(26.8 \pm 13.8$ days $)$ was significantly longer than $\mathrm{TH}-(8 \pm 4.4$ days; $P<0.0001)$. There was no significant difference in severity scores between $\mathrm{TH}+$ and TH , APACHE III $(21 \pm 10.3$ vs. $19.4 \pm 8.7)$ or ISS $(24 \pm 14$ vs. $21 \pm 11.2)$. $\mathrm{TH}-$ had a significantly higher mortality rate than $\mathrm{TH}+(17.6 \%$ vs. $9.7 \%, P=0.004)$.

Conclusion: We found that infections are the most common cause of thrombocytosis. This latter appears to be associated with lower mortality but longer duration of ICU stay. It may serve as an additional variable in predicting outcome of TBI patients. Thrombocytosis is not associated with a significant risk for thromboembolic events.

\section{P 022}

\section{In vitro effects of histamine and glutamate on the function of Aquaporin-4 in astrocytes}

P. de Vooght ${ }^{\star}$, S. Mertens ${ }^{\dagger}$, A. Houben ${ }^{\dagger}$, R. Nuydens ${ }^{\dagger}$, R. De Jongh*, T. Meert ${ }^{\dagger}$

*ZOL, Department of ICU, Genk; 'Janssen Pharmaceutica, Beerse, Belgium

Background and objective: Thirteen human water channels, aquaporin (AQP) 0-12, allow for rapid bi-directional water transport across cell membranes. In the brain, AQP4 is the most abundant water channel, mainly expressed at the endfeet of astrocytes facing the glia limitans and blood vessels. It plays an important role in brain water homeostasis and may be implicated in the formation and resolution of brain oedema. After traumatic brain injury (TBI) several neuronal mediators, such as histamine and glutamate, are released in the brain and may interfere with water homeostasis. The aim was to investigate the effects of histamine and glutamate on in vitro hypotonic-induced astrocyte swelling.

Methods and materials: An in vitro model for brain oedema was developed by applying a hypotonic shock to induce swelling of human 1321-N1 astrocytoma cells, either control or AQP4 transfected. The effects of pretreatment of these cells with histamine or glutamine on hypotonic-induced cell swelling and on cellular location of AQP4 were investigated. In addition, intracellular calcium $\left(\mathrm{Ca}^{2+}\right)$ concentrations after application of these mediators were quantified.

Results: Histamine and glutamate induced a dose-dependent inhibition of cell swelling on hypotonic shock. Concomitant with these effects on cell volume regulation, both mediators induced an increase of the intracellular $\mathrm{Ca}^{2+}$ concentration and the 
translocation of AQP4 from the plasma membrane to the cytoplasm, as quantified by immunocytochemistry.

Conclusion: These results indicate that histamine and glutamate inhibit hypotonic-induced cell swelling. Internalization of membrane-associated AQP4 reduces the water flux capacity of astrocytoma cells. A putative intracellular signalling pathway, increased intracellular calcium, might be responsible for altering the water channel's location and might explain the similarity of effects elicited by histamine and glutamate. However, confirmation experiments for this hypothesis are necessary.

\section{STROKE/SUBARACHNOID HAEMORRHAGE}

\section{P 023}

\section{Endovascular treatment of ruptured intracranial} aneurysms using the Guglielmi Detachable Coil in the elderly

M. Souissi, W. Trabelsi, M. Soussi, N. Baffoun, K. Baccar, C. Kaddour

National Institute of Neurology, Department of Anesthesia and Intensive Care, La Rabta, Tunisia

Background and objective: Little information is available about management of elderly people by endovascular treatment of ruptured intracranial aneurysms. The aim was to assess clinical outcomes in elderly patients treated by embolization for ruptured intracranial aneurysms.

Methods and materials: An open prospective observational study. Inclusion: Patients with subarachnoid haemorrhage after spontaneous rupture of intracranial aneurysm treated exclusively by embolization using Guglielmi detachable coils and age $>60$ yr. Collected data: medical history, clinical and laboratory examinations, World Federation of Neurosurgeons Grade (WFNS) and Fisher's Classification. Neurological outcome was assessed by modified Rankin scale [Bonita Stroke 1988] and the Glasgow Outcome Scale (GOS). Study period: July 2006October 2006.

Results: Eight patients (sex ratio $=5 / 3$ ), mean age $65 \pm 4.5 \mathrm{yr}$ (61-74) were included. Five patients were WFNS Grade I-II, two patients WFNS Grade III and one patient WFNS Grade IV. Complete obliteration of the aneurysm was successful in seven cases and partial $(80 \%)$ once. Aneurysm thrombosis complicated two procedures and was fatal for both (respectively, 3 and 5 days after embolization) due to massive ischaemia (aneurysm of the internal carotid artery) and refractory intracranial hypertension (aneurysm of the anterior communicating artery). Those two patients were, respectively, WFNS Grade / Fisher Classification: III/3 and II/2. The patient with WFNS Grade IV had a successful uncomplicated procedure 14 days after the initial insult and partial clinical recovery. He continued to improve and was discharged alive from hospital without major neurological disability (GOS: good, modified Rankin scale 2).

Conclusion: Endovascular coiling could be an efficient therapeutic tool. Incidence and outcome of procedure complications is still to be determined. Strategy in patients with high WFNS grade is certainly to try embolization because surgery would be very risky. Appropriate management timing is still to be determined.

\section{P 024}

Is there any difference in outcome after embolization of ruptured intracranial aneurysms between anterior and posterior location?

M. Souissi, W. Trabelsi, Z. Zaghdoudi, K. Baccar,

N. Baffoun, C. Kaddour

National Institute of Neurology, Department of Anesthesia and Intensive Care, La Rabta, Tunisia
Background and objective: We evaluated possible differences in short term outcome between anterior and posterior ruptured aneurysms treated exclusively by embolization.

Methods and materials: An open prospective clinical study between July-October 2006. Included were consecutive patients that underwent embolization for ruptured aneurysms. Data collected were patient characteristics, neurological examination, CT-Scans, WFNS Grade, Fisher modified classification, aneurysm characteristics, vasospasm, duration, medications, haemodynamics and eventual complications during the procedure. Main outcomes of interest were specific morbidity and mortality directly related to embolization and during hospitalization. After endovascular treatment, patients were sent to our ICU.

Results: Thirty two patients (sex ratio 15/17) were examined. Anterior circulation aneurysms were present in 24 patietns, the commonest location being the anterior communicating artery $(n=13)$. Posterior circulation abnormalities were seen in eight patients . Five patients died in hospital and two were discharged alive but with compromised short-time courses. No statistical difference was observed between anterior and posterior locations concerning medical history, WFNS Grade or characteristics of aneurysms. Vasospasm was correlated with duration of procedure $(P=0.01)$. Seven direct complications of embolization treatment were seen in seven patients (five aneurysm thrombosis, one procedural aneurysm rupture, one case of unreachable aneurysm). Thrombosis occurred despite 5000IIU heparin bolus given systematically after first coil leach, heparin titrated according to PTT. Multivariable statistics were used. Aneurysm size was the sole favouring factor for thrombosis $(P=0.013)$. Thrombosis was fatal in at least two cases and was an independent mortality risk factor. Posterior circulation produced one complication with thrombosis of a $10 \mathrm{~mm}$ aneurysm of the basal artery tip. Aneurysm rupture during embolization occurred in a 41 y.o female with a Grade I Fisher 1 subarachnoid haemorrhage due to a $3 \mathrm{~mm}$ pericallosum aneurysm. Rupture occurred after the 1st coil leach. The procedure was completed successfully and the patient experienced a transient neurological defect.

Conclusion: There is a need to study more patients. Aneurysm thrombosis can be life threatening. Anticoagulation strategy needs to be reviewed.

\section{P 025}

Management after rupture during embolization of aneurysms with Guglielmi electrodetachable coils

M. Souissi, W. Trabelsi, K. Baccar, N. Baffoun, M. Soussi, C. Kaddour

National Institute of Neurology, Department of Anesthesia and Intensive Care, La Rabta, Tunisia

Background and objective: Subarachnoid haemorrhage (SAH) may complicate rupture of aneurysms and be responsible for severe intracranial hypertension. We describe one case of aneurysmal perforation during embolization with Guglielmi electrodetachable coils (GDCs). 
Methods and materials: We studied patients treated by embolization after spontaneous intracranial aneurysm rupture. Included were those where embolization was complicated by rupture of an aneurysm during the obliteration procedure. The rupture was ascertained by extravasation of contrast medium.

Results: In the period from July to October 2006, 32 patients received embolizations for 32 aneurysms. One patient presented with a rupture during the embolization: She was a 41 y.o female who had come to our institution's emergency department suffering from an acute headache, nausea, vomiting and mild meningism. She had no neurological defect (WFNS Grade I). CT-scan showed a mild SAH (Fisher Class 1). Angiography followed confirming the presence of a $3 \mathrm{~mm}$ ruptured pericallosum aneurysm. During the embolization procedure, sudden haemodynamic instability (bradycardia, unstable blood pressure) was noticed and rupture of the aneurysm confirmed by extravasation of contrast medium. This complication occurred during placement of the first coil. The procedure continued successfully and the aneurysm was completely obliterated by three coils. CTscan performed immediately after the end of the procedure showed no massive cerebral haemorrhage (Class 2 Fisher). The patient was thereafter transferred to our ICU where she was extubated. She developed a transient neurological defect (right hemiparesis). She was discharged alive without any disability.

Conclusion: Aneurysmal perforation during embolization seems to be a rare event. In our case it did not cause much damage, but clinical severity is variable and far from being predictable. Rebleeding can result in severe intracranial hypertension and ultimately brain death.

\section{$P 026$}

The correlation of anaesthetic factors and postoperative complications in patients with ruptured cerebral aneurysm

P. Akavipat, K. Damrongbul, S. Pannak

Prasat Neurological Institute, Bangkok, Thailand

Background and objective: To determine the correlation of anaesthetic factors with postoperative complications in patients with ruptured cerebral aneurysm.

Methods and materials: This retrospective study was undertaken from chart and anaesthetic record reviews in order to analyse the correlation between anaesthetic related data; sex, age, ASA classification, preanaesthetic problems, Glasgow Coma Score (GCS), aneurysmal location, anaesthetic technique, fluid and medications, clinical occurrence during anaesthesia, patient position and anaesthetic time and $72 \mathrm{~h}$ postoperative complications. Twenty-nine patients with postoperative complications and 62 patients without complications and who had a ruptured cerebral aneurysm were randomized and recruited into this study. With these data, 2 test and a multiple logistic regression procedure were employed.

Results: From the factors investigated, the following were associated with postoperative complications: (1) ASA physical status 3-5, (2) GCS 3-12, (3) hypotension and (4) colloid administration. The correlation $(Y)$ was shown with this equation: $\mathrm{Y}=1.6$ ASA physical status $(3-5)+3.07$ GCS $(3-12)+1.19$ hypotension +2.29 colloid administration -5.66 .

Conclusion: The results of this study can help identify patients with a higher risk of developing postoperative complications. The study of relevant features of each factor should be further performed especially the necessity of colloid fluid administered.

\section{P 027 \\ Prospective analysis of hypervolaemic hypertensive haemodilution therapy in clipped aneurysms}

M. Celik, A. Elmaci, B. Betül, F. Bölükbaby, K. Tayyibe

Goztepe Education and Research Hospital, Department of Anesthesiology and Reanimation, Istanbul, Turkey

Background and objective: At present, a diversity of many approaches to treating vasospasm has been developed, among them hypertensive hypervolaemic haemodilution ( $3 \mathrm{H}$-therapy ) offers certain advantages.

Methods and materials: Ten patients who underwent early craniotomy for aneurysm clipping surgery had undergone hypertensive hypervolaemic haemodilution (3H-therapy) under guidance of systemic haemodynamics, by using a central venous catheter. Hypervolaemia was obtained by using volume expansion. Dopamine was used if necessary to obtain hypertension. The goal for haemodilution was a haematocrit of 0.30 to 0.33 . Daily volume infused, urinary output,TCD ( Vmax, Pi ) and serum electrolyte levels were recorded. CRP and blood glucose were measured also. Adverse effects were reported (e.g. pulmonary oedema or tachycardia) also and any clinical finding of vasospasm.

Results: The 3-H therapy was maintained for about thirteen days. The mean GCS was 14, Hunt-Hess Classification was 1.8, Fisher grade was 2.2. preoperative TCD (Vmax) was 120 . After the aneurysm was secured $13800 \mathrm{~mL}$ crystalloid per day was infused and $11750 \mathrm{~mL}$ urine output was recorded. Mean CVP values were $9 \mathrm{mmHg}$, MAP $106 \mathrm{mmHg}$, haematocrit $31 \%$, respectively. CRP and Vmax parameters were also decreased. Conclusion: It should be emphasized that $3 \mathrm{H}$-therapy may be used in operated aneurysms, by thoroughly monitoring the parameters of central haemodynamics, blood coagulation system, cerebral circulation and intracranial pressure.

\section{P 028}

\section{Bedside CBF-monitoring in patients with subarachnoid haemorrhage}

E. Keller, R. Mudra ${ }^{\dagger}$, C. Muroi ${ }^{\star}$, O. Yaldizli, J. Fröhlich

*Universitätsspital Zürich, Department of Neurosurgery,

Neurointensive Care Unit; 'Institute for Biomedical Engineering, University of Zürich/ETH; "Laboratory for Electromagnetic Fields and Microwave Electronics, ETH, Zürich, Switzerland

Background and objective: Radiographic cerebral vasospasm (CVS) after subarachnoid haemorrhage (SAH) may occur in the absence of clinical deficit and vice-versa. A practical method for measuring cerebral blood flow (CBF) at the bedside in the ICU is still lacking. New techniques combining near infra-red spectroscopy (NIRS) and indocyanine green (ICG) dye dilution to estimate cerebral haemodynamics have recently been developed. The aims were: (1) to establish the new method in the clinical setting and (2) to estimate its value in detection and treatment of CVS.

Methods and materials: Four NIRS optodes were placed bilaterally on the forehead (extended prototype, Oxymon system, Artinis, Njimegen). After daily central venous injections of $0.5 \mathrm{mg} \mathrm{kg}^{-1}$ ICG dye dilution curves were recorded and regional values for the mean transit time (mttlCG), cerebral blood volume (CBV) and CBF were quantified. Data are compared with $U$-test. Results: No side-effects occurred. In all, 135 ICG injections in 26 patients were performed. In three patients no values of cerebral haemodynamics could be calculated because of artifacts. Mean values obtained over both hemispheres were for mttICG $9.4+2.5 \mathrm{~s}(n=182)$, for CBV $3.4+2.1 \mathrm{~mL}$ per $100 \mathrm{~g}(n=177)$ and for CBF $22.6+14.5 \mathrm{~mL}$ per $100 \mathrm{~g} \mathrm{~min}^{-1}(n=177)$. In all, 9 of 
23 patients developed symptomatic CVS confirmed with angiography. Mean values for CBV and CBF were significantly lower with the presence of CVS (for CBV $3.1+2.1$ vs. $4.5+2.4 \mathrm{~mL}$ per $100 \mathrm{~g} ; P=0.0145$ and for CBF $19.6+14.3$ vs. $29.9+15.5 \mathrm{~mL}$ per $\left.100 \mathrm{~g} \mathrm{~min}^{-1} ; P=0.0029\right)$. There were no significant differences between mttICGmean with and without CVS.

Conclusion: The new technique is a promising method for serial bedside CBF measurements in the ICU. It has the advantage of being non-invasive and does not require the patient to be transported. The technique could be a powerful tool in detection and treatment of CVS. Investigations in a larger set of patients are needed to evaluate its diagnostic accuracy in detection of CVS.

\section{P 029}

\section{Treatment of resistant fever with local cerebral cooling}

B. J. Gaida*, O. Oezguer Yaldizli*, S. Mink*, C. Muroi*, R. Mudra ${ }^{\dagger}$, J. Fröhlich

*UniversitätsSpital Zürich, Department of Neurosurgery, Neurointensive Care Unit; ${ }^{\dagger}$ Institute for Biomedical Engineering, University of Zürich/ETH; 'Laboratory for Electromagnetic Fields and Microwave Electronics, ETH, Zürich, Switzerland

Background and objective: Treatment of fever is an important tool to minimize the extent of neuronal damage in neurointensive care patients. Conventional measures are not effective enough in critically ill neurological and neurosurgical patients.

Methods and materials: Fever treatment included standard management (acetaminophen, novaminsulfone, alcohol washing, ice packs) plus, if brain temperature was still $>37.8^{\circ} \mathrm{C}$ after $2 \mathrm{~h}$, a cooling head and cervical wrap connected to a conditioning unit (CSZ Blanketrol, Cincinnatti, Ohio). Brain temperature was continuously recorded via a thermistor embedded in the ventricular drainage (Neurovent, Raumedic, Münchberg, Germany). Temperature values from the brain (Tbrain) and blood (Tblood) (arterial line) were analysed hourly for $6 \mathrm{~h}$ after wrap cooling was started (hours 0-6). Comparisons of temperature values were performed using paired $t$ tests.

Results: In all, 266 temperature values with 19 episodes of intractablefever in six consecutive patients with aneurysmal subarachnoid haemorrhage were analysed. Tbrain and Tblood after $6 \mathrm{~h}$ of wrap cooling decreased significantly from Tbrain $38.5+0.6$ and Tblood $38.2+0.6$ to $37.5+0.4$ and $37.4+0.5$ $(P<0.0001$ for both). Brain temperatures were significantly higher compared to blood temperatures at hour 0 (mean difference of $0.3^{\circ} \mathrm{C} ; P=0.0003$ ), at hour 6 (mean difference $0.2^{\circ} \mathrm{C} ; P=0.0006$ ), as well as during the entire study period (mean difference $0.17^{\circ} \mathrm{C} ; \mathrm{P} \leqslant 0.0001$ ).

Conclusion: (1) Local cerebral cooling with a wrap, covering head and neck is feasible and may be effective in lowering brain temperature in patients with fever refractory to conventional treatment. (2) Monitoring of brain temperature with thermistors in the ventricular system or in the deep brain parenchyma is essential to study the effectiveness of new devices for selective cerebral cooling. (3) In the present study head and neck were wrapped. As suggested with model simulations, further clinical studies will show up whether cooling with a neck collar alone will be effective enough to control fever.

\section{$P 030$}

\section{The risk factors among patients with different lacunar stroke develoment mechanisms}

O. Vinogradov, A. Kuznetsov

National Centre of Cerebrovascular Disorders, Moscow, Russian Federation
Background and objective: The most frequent reasons for development of lacunar stroke (LS) are: hypertonic microangiopathy, atherosclerotic microangiopathy, embolism from cardial or arterial nidus. The purpose of this study was detection of the risk factor (RF) distinctions among patients with different mechanisms of development of LS.

Methods and materials: We examined 211 patients with acute LS. On the basis of the LS development mechanism the patients were divided into three groups: consequences of hypertonic microangiopathy (group I), 99 patients (46.9\%); atherosclerotic microangiopathy (group II), 80 patients (37.9\%); and embolism (group III), 32 patients (15.2\%). We used RF analysis, MRI, echocardiography, carotid duplex sonography, transcranial Doppler sonography with microemboli detection and biochemical blood tests.

Results: Essential hypertension in group I was present in 96 cases (96.9\%) and this was statistically different from group II, 67 patients $(83.7 \%)$ and III, 26 patients $(81.3 \%), P<0,01$. Diabetes mellitus in group II was present in 24 cases (30\%), in group III, 12 cases (37.5\%), $P>0.05$ and in group I, 13 cases $(13.1 \%)$, $P<0.01$. Hyperlipidaemia in group II was present in 75 cases $(93.8 \%)$, in group III, 28 cases (87.5\%), $P>0.05$ and in group I, 43 cases $(49.5 \%), P<0.01$. No distinctions between the groups were detected in such RF as smoking, alcohol abuse, liquefaction and low physical activity.

Conclusion: The RF distribution among the patients with LS confirms the heterogeneity of this pathogenetic sub-type of ischaemic stroke.

\section{P 031}

\section{Outcome after haemorrhagic transformation of cerebral infarction}

E. Batishcheva, A. Kuznetsov, O. Vinogradov,

M. Gabotinskaya

National Centre of Cerebrovascular Disorders, Moscow, Russian Federation

Background and objective: Haemorrhagic transformation (HT) of cerebral infarction is a relatively common event. The incidence of symptomatic HT averages $0-6.8 \%$ and asymptomatic HT $2.9-36.8 \%$. There is an opinion that HT predicts unfavourable outcome of ischaemic stroke. The aim was to estimate the structure of HT and to determine the influence of HT on stroke outcome.

Methods and materials: In all, 128 patients with ischaemic stroke were examined, 64 patients with HT and 64 without HT. Patients in both groups were similar in age, gender, sub-type of ischaemic stroke, severity of stroke at onset, size of infarction. CT and MRI, Glasgow Coma Score. NIHSS and modified Rankin scale were applied on the 1st, 2nd and 21st hospitalization day.

Results: Asymptomatic HT was diagnosed in 47 patients (73.4\%) and symptomatic HT in 17 (26.6\%). Haemorrhagic infarction of the first sub-type $(\mathrm{HI}-1)$ presented in 27 patients $(42.2 \%), \mathrm{HI}-2$ in $16(25 \%)$, parenchymatous haematoma of the 1st type $(\mathrm{PH}-1)$ in $12(18.7 \%)$ and $\mathrm{PH}-2$ in 9 (14.1\%). $\mathrm{HI}-1$ and $\mathrm{HI}-2$ predominated in patients with asymptomatic $\mathrm{HT}(80.9 \%)$ while $\mathrm{PH}-1$ and $\mathrm{PH}-2$ predominated in symptomatic HT (70.6\%). The early outcome was better in patients with asymptomatic HT than in the control group $(P<0.05)$. There were no differences in outcomes between patients with symptomatic HT and patients of the control group.

Conclusion: HT is not a predictor of unfavourable outcome of ischaemic stroke. Asymptomatic HTs, consisting mainly of $\mathrm{HI}-1$ and $\mathrm{HI}-2$ can predict favourable clinical outcome. They can be considered as a marker of successful reperfusion. 


\section{P 032}

Initial leucocytosis and hyponatraemia occuring after aneurysmal subarachnoid haemorrhage - do they predict poor outcome?

G. T. Taleska, V. D. Durnev, V. M. Mircevski,

M. S. Soljakova, Z. S. Suplinoski, T. T. Trajkovska,

Lj. A. Agai, S. A. Ackovska, V. D. Davceva,

Lj. M. Momirovska, M. N. Naumovska

University Clinical Center, Clinic of Anaesthesiology Reanimation and Intensive Care, Skopje, Macedonia

Background and objective: The blood clot size on admission remains the only factor that increases risk of cerebral vasospasm after SAH. Recently, it was reported that initial leucocytosis and hyponatraemia may be risk factors for development of cerebral vasospasm.

Methods and materials: We reviewed data of 45 patients admitted for SAH in 2006-2007. There were 14 males and 31 females. The end-point of a poor outcome was overall mortality. Results: Overall mortality was $48.88 \%$. Sex distribution of mortality rate was males/women $8.88 \% / 40.0 \% \quad(P<0.05)$. Symptomatic vasospasm occurred in $51.1 \%$. We found three predictors of vasospasm, namely Hunt and Hess grade 3, Fisher grade 3 and leucocytosis $>15 \times 109$ per $L$ up to the third day of admission. Initial leucocytosis was associated with greater mortality: discharged/deceased $18.2 \% / 50 \%(P<0.05)$. No correlation between admission leucocytosis and fever was observed nor between later leucocytosis (after day 5) and vasospasm. The examination of correlation of initial hyponatraemia $\left(<135 \mathrm{mmol} \mathrm{L}^{-1}\right)$ on third to fifth day of admission with mortality rate did not show statistical significance: discharged/deceased $20 \% / 24.4 \%$. Severe initial hyponatraemia $\left(<125 \mathrm{mmol} \mathrm{L}^{-1}\right)$ occurred less frequently: discharged/deceased $11.1 \% / 17.8 \%$.

Conclusion: This study reveals that leucocytosis increases the risk of developing vasospasm but does not prove that initial hyponatraemia has a direct contribution to the pathogenesis of ischaemic complications following $\mathrm{SAH}$.

\section{P 033}

Neurosurgical site infections in patients treated with long-term hypothermia and barbiturate coma after subarachnoid haemorrhage and early clipping

O. Yaldizli ${ }^{\star}$, M. Seule ${ }^{\dagger}$, C. Muroi ${ }^{\star}$, M. Fortunati ${ }^{\star}$, E. Keller ${ }^{\star}$

*University of Zurich, Departement of Neurosurgery, Zurich; ${ }^{\dagger}$ Kantonsspital St Gallen, St Gallen, Switzerland

Background and objective: Long-term systemic hypothermia (LTH) and barbiturate may predispose to potentially life-threatening bacterial infections. Neurosurgical site infections (NSI), including incisional infections (IS) and external ventricular drain infections (EVDI) affect about $10-14 \%$ of patients following subarachnoid haemorrhage (SAH), craniotomy and ventriculostomy. The aim of the study was to evaluate whether NSI are more common in patients with LTH and barbiturate coma.

Methods and materials: This was an observational prospective cohort study in 100 consecutive patients (36 male, 64 female, mean \pm SD age $55 \pm 9.7$ ) between 1999 and 2006 following SAH and early clipping, treated with LTH (mean duration $169 \pm 104 \mathrm{~h}$ ) and barbiturate coma because of intracranial hypertension and cerebral vasospasm refractory to conventional treatment. CSF out of EVD were routinely analysed twice per week. Awound swab was taken only if clinical signs of wound inflammation occured.

Results: Follow-up data were available in 92 patients; 34 (37\%) had a favourable outcome (GOS 4-5), whereas 58 (63\%) remained severe disabled, in a vegetative state or were dead after $1 \mathrm{yr}$ (GOS 1-3). Seven of 76 patients with EVD (9.2\%) developed an EVDI (positive CSF culture plus elevated white blood cell count in CSF). The bacteria isolated from the CSF were gram positive cocci (including Staphylococcus aureus and coagulase negative staphylococcus) in six patients and gram positive rods in one patient. Five of the 100 patients developed clinical signs of a IS after craniotomy. In two of these five patients, pathogenic bacteria could be grown from the wound swab (Enterococcus sp., Citrobacter koseri).

Conclusion: Compared with previous data, there is no higher rate of EVDI and IS in patients with LTH and barbiturate coma. Larger observational studies are needed to specify the microbiological pattern of NSI in patients with LTH and barbiturate coma.

\section{P 034}

Statin and magnesium administration in patients after aneurysmal subarachnoid haemorrhage: a retrospective study

T. Kerz ${ }^{*}$, C. Beyer ${ }^{\star}$, I. Trapp*, R. Reisch*, A. Victor ${ }^{\dagger}$

*Johannes Gutenberg University Medical School, Department of Neurosurgery; 'Johannes Gutenberg University Medical School, Institute of Medical Biostatistics, Mainz, Germany

Background and objective: Simvastatin (S) decreases the incidence of vasospasm and delayed cerebral ischaemia (DCI) after aneurysmal subarachnoid haemorrhage (SAH). Magnesium $(\mathrm{Mg})$ could also reduce $\mathrm{DCl}$ after $\mathrm{SAH}$. We reviewed 100 patient charts before and after initiation of combined $\mathrm{S}$ and $\mathrm{Mg}$ therapy to determine the incidence of $\mathrm{DCl}$.

Methods and materials: All patients admitted to our institution with aneurysmal SAH from January 2007 were treated with simvastatin $20 \mathrm{mg}$ for three days, then $40 \mathrm{mg} \mathrm{day}^{-1}$ for 11 days. Concomitantly, magnesium sulphate $80 \mathrm{mmolday}^{-1}$ was given. When mean arterial blood pressure fell to $<80 \mathrm{mmHg}$ with $\mathrm{Mg}$-administration, only $\mathrm{S}$ was given. Charts were reviewed for $\mathrm{DCl}$ as primary outcome parameter, defined as a new hypodense lesion on computed tomography and angiographic confirmation of vasospasm. Mortality was a secondary outcome parameter. Patients treated without $\mathrm{S}$ and $\mathrm{Mg}$ then served as the control group. Statistical analysis was performed using SPSS v12.0.

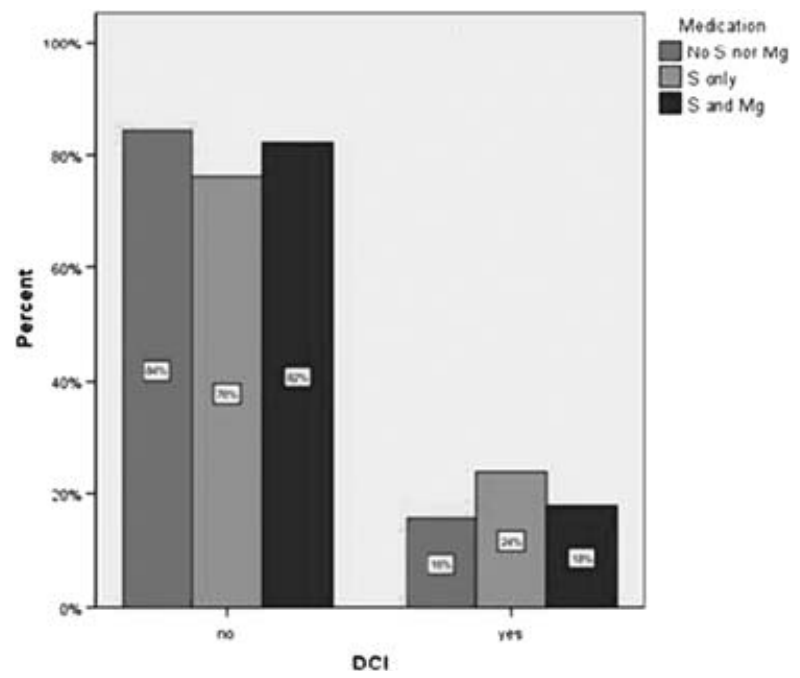

Figure 1. 
Results: In all, 28 patients were treated with $\mathrm{S}$ and $\mathrm{Mg}, 21$ patients received only $S$ and 51 patients served as controls. Median age was 54.9yr (interquartile range 46.9-64.2). There were 64 females. No differences were found between groups in Hunt \& Hess, GCS, WFNS and Fisher scales, aneurysm localization or rate of external ventricular drainage. There was a trend for more comatose patients in the no $\mathrm{S}$ or $\mathrm{Mg}$ group. There was only a slight difference in $\mathrm{DCl}$ between groups (Fig. 1), but 14 day-mortality was reduced in $S$ patients. Patients receiving $S$ and $\mathrm{Mg}$ made an intermediate group.

Conclusion: No significant differences were found between groups. While $\mathrm{DCl}$ was slightly reduced in patients without $\mathrm{S}$ or Mg $(P=0.61)$, the mortality rate was lower for $S$ patients $(P=0.14)$. Whether this is due to uncontrolled factors (e.g. blood pressure differences, time differences) or to other effects of statins (e.g. pleiotropic effects) remains unclear.

\section{P 035}

Incidence of spontaneous intracerebral haemorrhage (SICH) and associated risk factors in a Portuguese university hospital case study

S. C. F. Torres, J. M. Mourão, C. M. P. Dias

Hospital São João, Department of Anaesthesiology, MTS, Portugal

Background and objective: Recently we have noticed an increase in the number and severity of admissions to our emergency department due to SHIC. The purpose of this study was to analyse clinical characteristics of patients admitted with $\mathrm{SICH}$ in a University Hospital.

Methods and materials: We retrospectively reviewed all medical records of patients admitted with stroke during first two quarters of 2006 and 2007 and selected those with SICH diagnosed by CTscan. Statistical analysis was performed using SPSS.

Results: Of the 247 patients selected, $118(47 \%)$ were admitted in 2006 and $129(52 \%)$ in 2007. Patient characteristics were similar. Although ICU admission and mortality increased during 2007 we did not find a statistical difference. Hypertension $P<0.05$ and anticoagulant therapy $P<0.05$ were the most frequent risks factors in both groups.

Conclusion: Despite our impressions, hospital admissions for $\mathrm{SICH}$ were not statistically different in these periods. As in other studies hypertension and anticoagulation were the most important risks factors associated with $\mathrm{SICH}$.

\section{P 036}

\section{Spontaneous subarachnoid haemorrhage (SSAH) in} Portugal: incidence and outcome (2003-2005)

C. Dias, J. Oliveira, C. Basílio, A. Cerejo

Hospital S. Joao, Department of Intensive Care - Neuro ICU, Porto, Portugal

Background and objective: SSAH is a serious neurological condition with poor outcome and important socio-economic impact. The aim was the evaluation of incidence and outcome of SSAH in Portugal between 2003-2005.

Methods and materials: We retrospectively studied 2306 medical records with diagnosis code 430 (ICD9-CM) from a national DRG database of 60 hospitals. Incidence rate was based on census information and statistics were performed with SPSS.

Results: We found an incidence of $7 / 1001000$ inhabitants per year. SAH occurred predominantly during winter and spring, showed less incidence in the north of Portugal and affected mostly females between 40 and $75 \mathrm{yr}$. Global mortality rate was $21 \%$. In all, 1276 patients (55\%) were submitted either to surgical or endovascular procedures and mortality rate was $12 \%$; $36 \%$ had aneurysm clipping and $20 \%$ had endovascular treatment. In all, $521(22 \%)$ of severely affected patients were admitted to ICU and mortality was $44 \%$.

Conclusion: Overall data of SSAH incidence and outcome in Portugal was found to be within the average rate of other Western countries.

\section{P 037}

Serum total and free cortisol concentrations after subarachnoid haemorrhage - no evidence for adrenal insufficiency (Al)

J. S. Bendel, T. S. Koivisto, J. Rinne, I. Vauhkonen,

E. Ruokonen, A. Uusaro

Kuopio University Hospital, Department of Anaesthesiology and Intensive Care, Kuopio, Finland

Background and objective: Recent studies suggest a crucial role of glucocorticoid metabolism in patients with traumatic brain injury and subarachnoid haemorrhage $(\mathrm{SAH})$. SAH may affect the hypothalamo-pituitary axis by direct compression of the aneurysm, blood clot/aneurysm, elevated intracranial pressure, vasospasm or surgical procedures. We measured serum concentrations of baseline and stimulated total and free cortisol consentrations in acute $\mathrm{SAH}$ during the first week and at 3 months after bleeding.

Methods and materials: Patients over 18yr scheduled for elective surgical aneurysm treatment $(n=16)$ and patients with SAH $(n=30)$ were included. Patients with corticoid treatment were excluded. During the first week, serum total and free cortisol was measured daily and a short ACTH-stimulation test was performed twice. At 3 months after bleeding, the measurements were repeated. We defined adrenal insufficiency as serum free cortisol concentrations under $22 \mathrm{nmol} \mathrm{L}^{-1}$ or $<55 \mathrm{nmol} \mathrm{L}^{-1}$, serum total cortisol concentrations $<414 \mathrm{nmolL}^{-1}$ or $<690 \mathrm{nmolL}^{-1}$ or as a blunted response to ACTH-stimulation test $\left(<248 \mathrm{nmol} \mathrm{L}^{-1}\right)$.

Results: Depending on criteria used, the percentage of patients who had adrenal insufficiency varied between $0 \%$ and $80 \%$. Serum total and free cortisol correlated well $(P<0.001)$. No difference between groups was seen in the response to the ACTH test $(P=0.11)$. There was no difference in serum total or free cortisol concentrations. Clinical grade, severity of bleeding, intracranial pressure, treatment modality, aneurysm location or hypoalbuminaemia did not affect the cortisol response.

Conclusion: SAH did not cause adrenal insufficiency during the first week or at 3 months. Serum free cortisol concentration was not helpful to identify patients at risk for adrenal insufficiency.

\section{P 038}

Neuroprotection by erythropoetin and Darbopoetin-alfa following experimental intracerebral haemorrhage

G. Grasso*, F. Meli*, F. Graziano*, V. Stagno*, F. Carletti ${ }^{\dagger}$, D. G. lacopino*

*Department of Clinical Neuroscience, Neurosurgical Clinic,

'University of Palermo, Department of Human Physiology,

Palermo, Italy 
Background and objective: Intracerebral haemorrhage $(\mathrm{ICH})$ is a devastating clinical syndrome for which no truly efficacious therapy has been yet identified. In preclinical studies, we have found that erythropoietin (EPO), administered in its recombinant form ( $\mathrm{rHuEPO}$ ), provides remarkable benefit in several models of brain and spinal cord damage. In this study we investigated the neuroprotective effects of EPO and its long-lasting derivative (Darbepoetin-alfa) in a experimental ICH model in the rat. Using these drugs, we asked whether a rHuEPO daily dose $\left(1000 \mathrm{U} \mathrm{kg}^{-1}\right.$ i.v.) or a Darbepoetin-alfa weekly dose $\left(10 \mu \mathrm{g} \mathrm{kg}^{-1}\right.$ i.v.), administered $5 \mathrm{~min}$ after $\mathrm{ICH}$ induction, provides any benefit as assessed by neurological outcome and histopathological investigations.

Methods and materials: Male Wistar rats were anaesthetized by an intramuscular injection of ketamine and placed in a stereotactic frame. A burr hole was performed by using a microdrill under microscopic control. A 30-G needle was introduced into the right striatum $(3 \mathrm{~mm}$ lateral to midline, $1 \mathrm{~mm}$ anterior to bregma, depth $3.5 \mathrm{~mm}$ below the surface of the skull) and $10 \mu \mathrm{L}$ of autologous blood, taken from the tail vein, injected over 1 min. All animals were sacrificed 15 days after $\mathrm{ICH}$ was induced.

Results: Treatment with rHuEPO and Darbepoetin-alfa largely prevented neurological deterioration and reduced neuronal damage as compared with saline-treated animals. Mean haemorrhage volume was also significantly decreased in $\mathrm{ICH}+\mathrm{rHuEPO}$ and Darbepoetin-alfa groups as compared to saline.

Conclusion: This study provides evidence that treatment with rHuEPO and its long-lasting derivative may be a beneficial therapeutic approach to reduce post-ICH morbidity.

\section{P 039}

\section{Coronary complication during post-trauma subarachnoid haemorrhage}

M. Souissi, M. Achour, W. Trabelsi, M. Soussi,

M. Maghouri, C. Kaddour

National Institute of Neurology, Department of Anesthesia and Intensive Care, La Rabta, Tunisia

Background and objective: The purpose was to assess the incidence of coronary complication during post-trauma subarachnoid haemorrhage (SAH).

Methods and materials: This was an open, double-blind, prospective study over a period of 15 months. Thirty-five patients with post-traumatic SAH were studied. Patients with a cardiovascular history, thoracic trauma and non-neurological coma were excluded.

Results: The mean age was $39 \pm 17 \mathrm{yr}$ and $85 \%$ had a Glasgow Coma Score $<8$. The mean ISS was $27 \pm 14$, and $6 \%$ and $54 \%$ had Fischer scales of 3 and 4 , respectively. There were electrocardiogram changes in $57 \%$ of patients. Repolarization abnormalities were the most frequent, occurring on the third day in $94 \%$ of cases. The mean \pm SD duration of these was $4 \pm 1$ days. Over the three measurements, total CPK was increased in $88 \%$ of patients, creatine kinase MB isoenzyme was increased in $65 \%$ of patients and Troponin lc was elevated in $34 \%$ of patients. The peak of Troponin lc occurred on the third day with an average of $0.113( \pm 0.338)$. The increase of Troponin Ic was associated with a poor CT scan grade. The elevation of Troponin Ic correlated with T-wave anomalies. There was no mortality related to a cardiac cause. The incidence of Troponin Ic and T-wave abnormalities could be considered predictive factors of mortality.
Conclusion: Repolarization abnormalities during posttrauma SAH are frequent in the first 3 days. They could reflect a myocardial lesion as it is indicated by the increase of Troponin Ic, which is associated with severe neurological injuries.

\section{P 040}

\section{Diagnostic marker of secondary ischaemia after subarachnoid haemorrhage}

G. K. Barcelos, F. Dailler, B. Renaud, G. Pardey,

A. Perred-Liaudet

Hospital Neurology and Neurosurgery Pierre Wertheimer, Department of Anaesthesia and Intensive Care, Lyon, France

Background and objective: To study the diagnostic value of jugular oxygen saturation $\left(\mathrm{SvJO}_{2}\right)$, jugular arterial lactate difference (DVAL), index of lactate oxygen (LOI) and glucose (OGI) for secondary ischaemia in subarachnoid haemorrhage (SAH) and their prognostic values.

Methods and materials: Forty patients with SAH aged 36-77 (mean 54)yr without haematoma and trauma were studied. Ischaemia was present by computed tomography $48 \mathrm{~h}$ after SAH onset in 24 patients; three patients died without ischaemia.

Jugular and arterial measurements were done, respectively, after $8 \mathrm{~h}$. Glucose and lactate were expressed in $\mathrm{mmol} \mathrm{L}^{-1}$. The Glasgow Outcome Score (GOS) was calculated leading to a separation of patients with poor outcome (3-4) and good outcome (1-2). U-test was used to determine significant differences between groups for prognostic and diagnostic purposes. Sensitivity and specificity were calculated using ROC curves.

Results: With this kind of off-line monitoring, $\mathrm{SvjO}_{2}$ was not useful for diagnosis nor for prognosis. At a cut-off $\leqslant 55 \%$ OGI was the earliest marker before CT scan with a sensitivity of $83 \%$ and specificity of $100 \%$. OGI was decreased from 8 to $36 \mathrm{~h}$ before hypodensity was seen. The cerebral response then lead to an increase of OGI to compensate. Cutoff values of DVAL were found to be $>0.15$ with sensitivity of $79 \%$ and specificity of $90 \%$. The lack of sensitivity of LOI was explained partly due to patients with peripheral lactate acidosis. At a cut-off of $>0.15$, DVAL was the best marker to predict poor outcome at 6 months with sensitivity and specificity of $79 \%$ and $90 \%$, respectively. OGI was not correlated to outcome.

Conclusion: These data will be very helpful for the management of $\mathrm{SAH}$ patients with particular attention to OGI monitoring.

\section{P 041}

\section{Is hypocholesterolaemia a risk factor for spontaneous intracerebral haemorrhage ?}

S. P. Paterniti, M. G. Maimone, F. P. Fiore, I. P. Paterniti, G. Gravina, C. M. Cambria

Policlinico Universitario Neurochirurgia, Neurosurgery, Department of Neurosciences, Messina, Italy

Background and objective: Some clinical and epidemiological studies published in the literature indicate that a low total cholesterol level (less than $160 \mathrm{mg} \mathrm{dL}^{-1}$ ) increases the risk of spontaneous intracerebral haemorrhage, especially in hypertensive aged males. Moreover, hypocholesterolaemia could be associated with greater mortality. The reasons for the 
association between hypocholesterolaemia and haemorrhagic stroke are still unknown: low circulating cholesterol might influence arterionecrosis, facilitating medial smooth muscle cell damage and pathological processes leading to haemorrhage in the intracerebral arterioles. Cholesterol lowering by statins might increase the risk of haemorrhagic stroke. However, the risk relationship between serum cholesterol level and spontaneous intracerebral haemorrhage was not confirmed by other studies; consequently controversy remains on the role of blood cholesterol as a cause of haemorrhagic stroke. The aim of this study was to investigate in our series the relationship between serum cholesterol level and spontaneous intracerebral haemorrhage and, secondarily, to assess the association with age and sex, arterial hypertension, other associated conditions and outcome according the Glasgow Outcome Scale.

Methods and materials: We selected the most recent 55 cases of spontaneous intracerebral haemorrhage. In all, 29 patients presented with low or very low total cholesterol level. There were 18 males and 11 females, 20 older than $65 \mathrm{yr}$ of age. Arterial hypertension was present in 10 cases and diabetes in 10. Four patients received anticoagulation or antiplatelet therapy. All suffered, on admission, from severe neurological conditions (GCS $<9$ with focal signs).

Results: In all, 10 patients died, 9 had severe or moderate disability, 10 obtained a good outcome.

Conclusion: We think that in our series there is a clear relationship between hypocholesterolaemia and incidence of spontaneous intracerebral haemorrhage but we have not found a strong correlation with age, sex, hypertension, clot location and outcome.

\section{P 042 \\ Apolipoprotein e polymorphism and outcome following aneurysmal subarachnoid haemorrhage}

S. P. Paterniti ${ }^{\star}$ I. R. Ientile ${ }^{\dagger}$, F. P. Fiore ${ }^{\star}$, M. G. Maimone*, I. P. Paterniti*, G. Gravina*, C. D. Caccamo ${ }^{\dagger}$,

C. M. Cambria*

${ }^{*}$ Policlinico Universitario Neurochirurgia, Neurosurgery,

Department of Neurosciences; 'BBiochemistry Department, Messina, Italy

Background and objective: Apolipoprotein $E$ (ApoE) is a polymorphic protein associated with plasma lipoproteins that mediates the cellular uptake of lipid complexes through specific receptors. The polymorphic gene for ApoE (APOE), located on the long arm of chromosome 19, has three common alleles e2, e3 and e4 that encode for three major isoforms of ApoE: E2, E3 and E4. ApoE plays an important role in neurobiology, especially in acute brain injury. The APOE genotype has been shown to be associated with outcome after spontaneous intracerebral haemorrhage and head injury. Recently, some studies investigated the APOE genotype influence on outcome after subarachnoid haemorrhage $(\mathrm{SAH})$ with conflicting findings being reported. Some authors found poorer outcomes in patients with the e4 allele whilst others found no association between APOE genotype and outcome. In other studies the association appears to be opposite where subjects with APOE4 had less severe haemorrhage and better outcome. In summary, the previous studies do not find a clear association between APOE genotype and outcome following $\mathrm{SAH}$. Demonstrating that the e4 allele predicts a negative clinical course would be of both clinical and speculative value. It could improve treatment and SAH would become a further possible target for the ongoing research on ApoE-based therapies. We began a study to test the possible influence of APOE polymorphism on outcome in patients with aneurysmal SAH.

Methods and materials: Various parameters were examined: age, condition on admission according to GCS and Hunt-Hess grading, amount of blood at CT scan according to Fisher grades, location of the aneurysm, arterial pressure, occurrence of rebleeding, vasospasm and ischaemia and outcome determined by GOS.

Results: Now we have only preliminary data that are not univocal; so it is difficult today to draw conclusions from our study.

Conclusion: The definitive results will be soon available and we will communicate them during the EuroNeuro Congress.

\section{P 043 \\ Epilepsy in venous stroke}

S. P. Paterniti, M. G. Maimone, F. P. Fiore, I. P. Paterniti, L. A. Levita, G. Gravina, C. M. Cambria

Policlinico Universitario Neurochirurgia, Neurosurgery,

Department of Neurosciences, Messina, Italy

Background and objective: Epileptic seizures frequently occur in cerebral venous thrombosis (CVT). However, in the literature there are few works reporting detailed descriptions of epileptic phenomena in this serious cerebrovascular disorder. To our knowledge there is only one study specifically dedicated to the subject. Here we report our experience.

Methods and materials: Reviewing our series of CVT we found that half of the patients presented with seizures. Sites of occlusion were dural sinuses nine cases and cortical veins three cases. Symptoms included increased intracranial pressure syndrome in 10 cases and focal deficit in six cases. There were focal or partial seizures in two cases, generalized seizures in nine cases and status epilepticus in one case. We found three cases of intraparenchymal lesions (haematoma or haemorrhagic infarction). All patients had single seizures in the acute stage. All received agents to control intracranial pressure, heparin anticoagulation, antiepileptic drugs (Phenobarbital and/or carbamazepina) and survived; 10 completely recovered, two presented with neurological sequelae. Antiepileptic drugs were progressively discontinued $1 \mathrm{yr}$ after the venous stroke. Early studies suggested that epilepsy was associated with poor outcome with mortality rates between $30 \%$ and $50 \%$. The recent series reports better results with an overall satisfactory outcome (mortality 5.5-30\%).

Results: In our series of venous stroke there is a high percentage of seizures $(50 \%)$. In our patients the involvement of cortical veins and the presence of cerebral ischaemic infarct or haemorrhage seem to be predisposing factors.

Conclusion: Our experience, although little, confirms the favourable outcome: full recovery was obtained in all cases. Some authors referred residual epilepsy in $25-31.5 \%$ of patients who had seizures at the acute stage but others reported a low incidence (9.5-14.3\%). We stress again that none of our patients presented at long-term follow-up residual or recurrent epilepsy. Pharmacological prevention could be limited to cases with early seizures. Long-term treatment is not necessary in the majority of patients. 


\section{DIAGNOSIS/IMAGING}

\section{P 044}

A case of maternal cerebral vasospasm in eclampsia

M. Souissi, M. Maghouri, W. Trabelsi, C. Kaddour

National Institute of Neurology, Department of Anesthesia and Intensive Care, La Rabta, Tunisia

Background and objective: The pathophysiology of the neurological complications attributed to preeclampsia/eclampsia remains unclear. Cerebral vasospasm is a rare but serious complication of eclampsia.

Methods and materials: We report on a $30 \mathrm{yr}$-old female who developed right-side hemiplegia after caesarean section at 30 weeks of gestation due to eclampsia. Detailed radiological investigations were carried out in order to confirm the diagnosis and follow the course of the condition.

Results: Initial brain MRI demonstrated high signal intensity lesion in bilateral occipito-parietal and left frontal lobes on T2weighted and FLAIR-weighted imaging. The MR angiography (MRA) showed diffuse vasospasm of intracranial vessels. Transcranial doppler ultrasonography showed an increased mean velocity in both side intracranial vessels. Two weeks later, follow-up cerebral MRA was performed, cerebral vasospasm had partially resolved with application of high-dose methyl prednisolone and nimodipine.

Conclusion: We suspected that cerebral vasospasm in eclampsia causes cerebral ischaemia, which leads to cytotoxic oedema and dysfunction of the blood-brain barrier and cerebral autoregulation.

\section{P 045}

\section{Magnetic resonance imaging findings in cerebral fat embolism: a case report}

M. D. Souissi, M. Soussi, M. Achour, C. Kaddour

National Institute of Neurology, Department of Anesthesia and intensive Care, La Rabta, Tunisia

Background and objective: Cerebral fat embolism (CFE) syndrome is a rare, but potentially lethal, complication of long bone fractures. Neurological symptoms are variable, and the clinical diagnosis is difficult.

Methods and materials: The purpose of this case study is to demonstrate the value of MR of the brain for diagnosis of fat embolism syndrome and the contrast between clinical evolution and MR findings.

Results: A 38 yr-old male with no medical history was admitted with right femoral and tibial fractures which were initially stabilized by traction. Then, $48 \mathrm{~h}$ later, he presented with acute respiratory distress and acute mental state changes with coma. $\mathrm{He}$ was transferred to the ICU where he was ventilated to maintain oxygenation and to stabilize vital signs. Examination revealed petechial eruption. Chest radiography showed bilatera diffuse pulmonary infiltrates. Opthalmoscopy showed papillary nodules. After respiratory stabilization, MR scan of the head performed 10 days after trauma revealed multiple high intensity signals throughout the white matter, the basal ganglia, the cerebellum and the brain stem. Evolution was marked by clinica recovery with no neurological sequelae. A repeat MR after 30 days showed total regression of all lesions.

Conclusion: High-intensity lesions in the brain on MR images may serve as an early-appearing and more-sensitive indicator of the diagnosis of fat embolism in the clinical context of long-bone injury without head trauma. There is no correlation with clinical evolution. CFE is a potentially reversible disease that can have a good outcome.

\section{P 046}

Tuberous sclerosis - a case report

M. Stoian, D. Chitac, S. Stoian, B. Bontas, S. Stoica

Medicine University Bucharest, Department of Internal Medicine, DR I Cantacuzino Hospital, Bucharest, Romania

Background and objective: Tuberous sclerosis complex or Morbus Bourneville-Pringle disease is an autosomal dominant phakomatosis, first described by Desiree-Magloire Bourneville in 1880. This rare genetic disorder is usually associated with a triad of seizures, mental retardation and cutaneous lesions. On radiological investigations, calcified subependymal hamartomas, uncalcified cortical tubers, or and radial white matter lines, are characteristic.

Case presentation: We report a case of a 28 -yr-old female known from birth with mental retardation admitted with a 4month history of important weight loss, abdominal pain, amenorrhoea and gynaecomastia. Her past medical history revealed epilepsy from $25 \mathrm{yr}$. The diagnosis of pregnancy was excluded by abdominal ultrasound and this showed ovarian polycystic disease and multiple tumours in the liver. CT scan showed subependymal astrocytoma with multiple calcified nodules, cortical tubers, pulmonary lymphangiomatosis, multiple renal cysts, hepatic hamartomas and ovarian cysts. Despite of late diagnosis, an improvement in quality of life was obtained.

Discussion: The disorder is characterized by multiple hamartomas in several organs, mainly located in brain, heart and kidney. The cognitive impairment is significantly correlated with the presence of bilateral tubers and the age of seizure onset. Subependymal giant cell astrocytomas and pulmonary lymphangiomyomatosis are serious complications. The long-term prognosis in this patient still remains uncertain in spite of time diagnosis. There is now the exciting possibility of drug therapy for some of the manifestations of the disease.

\section{P 047}

\section{Are carotid fatty plaque associated with ischaemic symptoms?}

L. Saba*, R. Montisci*, R.Sanfilippo*, G. Caddeo ${ }^{\dagger}$,

L. Pascalis ${ }^{\dagger}$, G. Mallarini ${ }^{\star}$

${ }^{*}$ Policlinico Universitario di Monserrato; ${ }^{\dagger}$ Ospedale San Giovanni di Dio, Cagliari, Italy

Background and objective: Atherosclerosis of the carotid arteries is one of the leading cause of stroke. Risk of plaque is correlated with the degree of stenosis but other factor are currently indicated as potential risk factors. The purpose of this study was to evaluate if the presence of carotid fatty plaque is significantly correlated with symptomaticity.

Methods and materials: Between January 2005 and April 2007, 93 patients were studied with MDCTA to assess carotid arteries and underwent afterwards carotid endarterectomy (CEA). We analysed the following characteristics in each patient: type of plaque (fatty, mixed or calcified), plaque morphology (regular vs. irregular) and presence of ulceration; we evaluated also degree of stenosis (according to the NASCET criteria). Plaque characterization and stenosis measurement were performed by two experienced radiologists. CEA was performed by two experienced vascular surgeons. We correlated MDCTA data with surgical results and with the presence or absence 


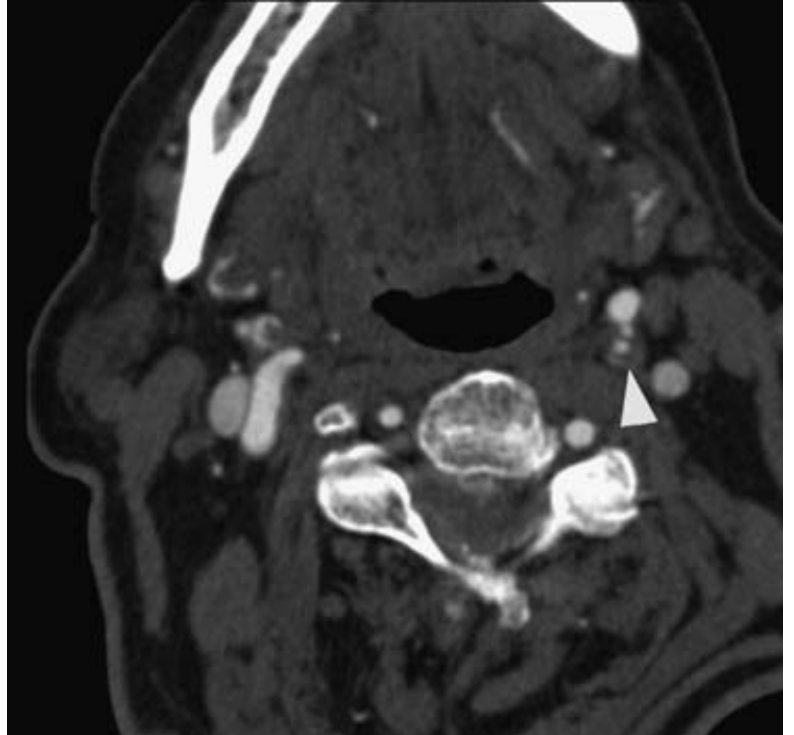

Figure 1 Axial CTA image, fatt Axial CTA image, fatty plaque (arrowhead).

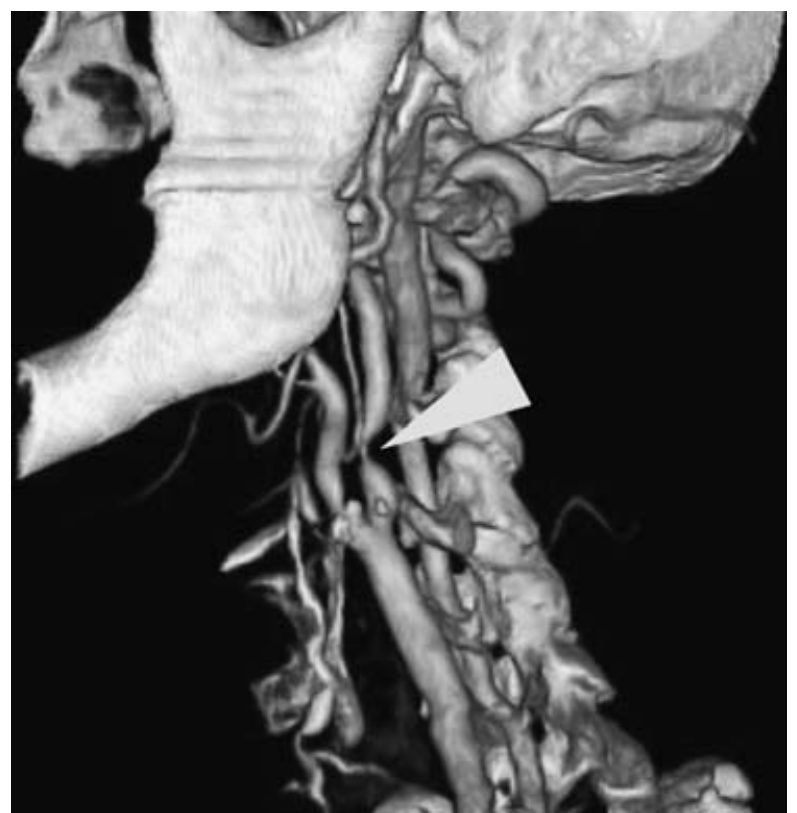

Figure 2. Axial CTA image, fatt Axial CTA image, fatty plaque (arrowhead).

of ischaemic events. Multivariate statistical analysis was provided to determine if an interaction existed between the presence of ischaemic episodes and specific plaque characteristics, and in particular the presence of fatty plaques as confirmed by surgery.

Results: A history of symptomatic ischaemic episodes was present in 59 patients. We found a positive correlation between the presence of fatty plaques and symptoms $(P<0.01)$. Moreover, we observed a statistically significant correlation between irregular morphology and symptoms $(P<0.01)$ and the presence of ulceration and symptoms.

Conclusion: The results of our study suggest that the presence of fatty plaque is a significant risk factor for ischaemic episodes. This type of plaque identifies as an 'unstable' plaque.

\section{P 048}

\section{Magnetic resonance imaging of stroke}

L. Saba*, R. Sanfilippo*, R. Montisci*, L. Pascalis ${ }^{\dagger}$,

G. Mallarini*

*Policlinico Universitario di Monserrato; ${ }^{\dagger}$ Ospedale San Giovanni di Dio, Cagliari, Italy

Background and objective: Strokes are a major cause of death and permanent disability. Stroke accounted for about one of every 15 deaths in the Western world in 2003. This condition occur when blood flow to a region of the brain is obstructed and may result in death of brain tissue. Stroke may result from localized cerebral ischaemia, intracerebral haemorrhage, subarachnoid haemorrhage of venous sinus thrombosis. The objectives of this work were: (1) to review and describe the magnetic resonance brain imaging characteristics of patients with Stroke; (2) to become familiar with the changing role of MRI in acute stroke triage; (3) to evaluate and discuss the new MR techniques for the study of stroke as dynamic perfusion, diffusion-weighted (DW) and MR angiography; and (4) to understand the various functional imaging modalities that can be applied in acute ischaemic stroke to identify functionally impaired, but morphologically preserved tissue (i.e. the penumbrae) and to distinguish it from irreversibly damaged tissue.

Methods and materials: Images we presented were acquired by using a with a 1.5-T superconducting magnet (Philips Intera). Patients head movements were restricted We used SE and TSE sequences, T1, T2 weighted with FLAIR sequences. Each exam was completed with of Gadolinium.

Conclusion: Magnetic Resonance demonstrates good sensitivity, specificity in identification of the extension of lesions in patients with Stroke. Diffusion-weighted images show optimal sensitivity and should be used as the modality of choice in stroke imaging; moreover this MR technique shows the infracted core in nearly every ischaemic stroke patients.

\section{P 049}

Carotid artery wall thickness and the type of plaque

L. Saba*, R. Sanfilippo*, R.Montisci*, G. Caddeo ${ }^{\dagger}$

L. Pascalis ${ }^{\dagger}$, G. Mallarini ${ }^{\star}$

*Policlinico Universitario di Monserrato; ${ }^{\dagger}$ Ospedale San Giovanni di Dio, Cagliari, Italy

Background and objective: Increased carotid artery wall thickness was reported to occur in an earlier phase of the atherosclerotic process. The aim of this work is to determine if carotid artery wall thickness (CAWT) evaluated by using multi-detector-row CT angiography (MDCTA) is associated with a specific type of plaque (fatty, mixed, calcified) development.

Methods and materials: In this retrospective study we analysed 116 patients by using a multi-detector row CT scanner. In each patient we measured CAWT and measurements were made with an internal digital caliper. We divided patients into three groups: in the first group (group A) were include patients with fatty plaque $(n=42)$, in the second group (group B) were included patients with mixed plaque $(n=35)$ and in the third group (group C) patients with calcified plaque $(n=39)$ and we made a statistical analysis to asses the CAWT difference in the three examined groups.

Results: Measurements of the distal common CAWT varied from 0.6 to $2.2 \mathrm{~mm}$. In the group A average CAWT was $1.101 \mathrm{~mm}$ $(0.226 \mathrm{SD})$ in group $B$ was $1.032 \mathrm{~mm}(0.256 \mathrm{SD})$ and in group $\mathrm{C}$ was 0.832 (0.184 SD). CAWT in group A patients showed statistical difference $(P<0.01)$ compared to group $\mathrm{C}$.

Conclusions: Results of our study suggest that an increased CAWT is associated with the fatty plaque development. This 
data should be considered by physician because it is well demonstrated that fatty plaques frequently ( 21 times the calcified plaques) determine TIA or strokes.

\section{P 050}

\section{Hypertension and dementia: a correlative study by} using magnetic resonance

L. Saba, R. Sanfilippo, R. Montisci, G. Mallarini

Policlinico Universitario di Monserrato, Cagliari, Italy

Background and objective: Vascular Dementia is a degenerative cerebrovascular disease that leads to a progressive decline in memory and cognitive functioning. This condition occurs when the blood supply carrying oxygen and nutrients to the brain is interrupted by a blocked or diseased vascular system. The purpose of this work was to evaluate whether the presence of diabetes is associated with vascular dementia.

Methods and materials: We retrospectively studies 74 patients (35 with and 39 without hypertension) (range age 54-82; average $67 \mathrm{yr}$ ). Each patient was studied with a 1.5-T superconducting magnet (Philips Intera). We used SE and TSE sequences, T1, T2 weighted with FLAIR sequences. Each exam was completed with of Gadolinium. Then we performed a statistical study to evaluate if the presence of diabetes is associated with vascular dementia.

Results: We observed a total of 39 patients with dementia and 39 without. There was a significant statistical association between the presence of hypertensio and vascular dementia identified by using magnetic resonance $(P=0.0096)$.

Conclusion: Results of our study indicate that the presence of hypertension is associated with presence of vascular dementia. Magnetic resonance imaging helps to precisely identify the white matter lesions by characterizing the extension of the disease.

\section{P 051}

Evaluation of metabolic diseases of CNS by using MR L. Saba*, G. Caddeo ${ }^{\dagger}$, G. Mallarini*

*Policlinico Universitario di Monserrato; ${ }^{\dagger}$ Ospedale San Giovann di Dio; Cagliari, Italy

Background and objective: CNS metabolic diseases, either congenital or acquired, are commonly caused by systemic disorders that interfere with the metabolism and result in neurological dysfunction. Many disorders primarily involving the liver, kidneys, heart, blood, and endocrine organs result in inconsistent supply of creatinine, glucose, and oxygen. MR spectroscopy can provide direct information on CNS metabolism. To aim was to review pathophysiology of CNS metabolic diseases.

Metods and materials: We used MR and multi-voxel spectroscopy imaging techniques to study CNS metabolic disease. We described potentialities and pitfalls of MR imaging techniques in the study of these syndrome, in particular MELAS (mitochondrial encephalomyopathy with lactic acidosis and stroke-like episodes) or hepatic encephalopathy induced by Cushings syndrome.

Results and Conclusion: Further study on absolute quantification of lactate in CSF will be helpful to establish the diagnosis, monitor the progression, replace the conventional invasive method of quantifying lactate in the CSF potentially, and evaluate the response to the treatment non-invasively. In fact lactate revealed by $1 \mathrm{H}-\mathrm{MRS}$ has good correlation with elevated lactate in serum and CSF. When lactate is produced in lesions of the brain and transferred to CSF, it could be detected by $1 \mathrm{H}-\mathrm{MRS}$

\section{P 052}

\section{Can the presence of vessel tortuosity cause} ischaemic accidents?

L. Saba, R. Sanfilippo, R. Montisci, G. Mallarini

Policlinico Universitario di Monserrato, Cagliari, Italy

Background and objective: Still remain an object of debated whether kinking or coiling of the internal carotid artery simply represents a morphological variation without clinical relevance. The purpose of our work was to evaluate whether the presence of tortuosity carotid arteries (kinking or coiling) is associated with symptomaticity.

Methods and materials: We retrospectively studied 189 consecutive patients by using Multi-Detector-row CT. A total of 378 carotid arteries were assessed for the presence of vessel tortuosity by two experienced radiologists in consensus. Each exam was performed by using a multi-detector-row scanner; contrast material was injected into ante-cubital vein and arterial phase images were obtained by using a delay time variable from 11 to 19 and by using a $4-6 \mathrm{~mL} \mathrm{~s}^{-1}$ flow rate. Statistic analysis was performed to determine if an independent interaction existed between the presence of vessel tortuosity and symptomaticity.

Results: We detected a total of 43 kinkings and 24 coilings. We observed a significant statistical correlation between the presence of kinking and symptomaticity (Fig. 1) ( $P$ value with Yates correction $=0.006$ and odds ratio 3.14), whereas we did not find a statistical correlation between coiling and symptomaticity $(P$ value with Yates correction $=0.041$ and odds ratio 1.55).

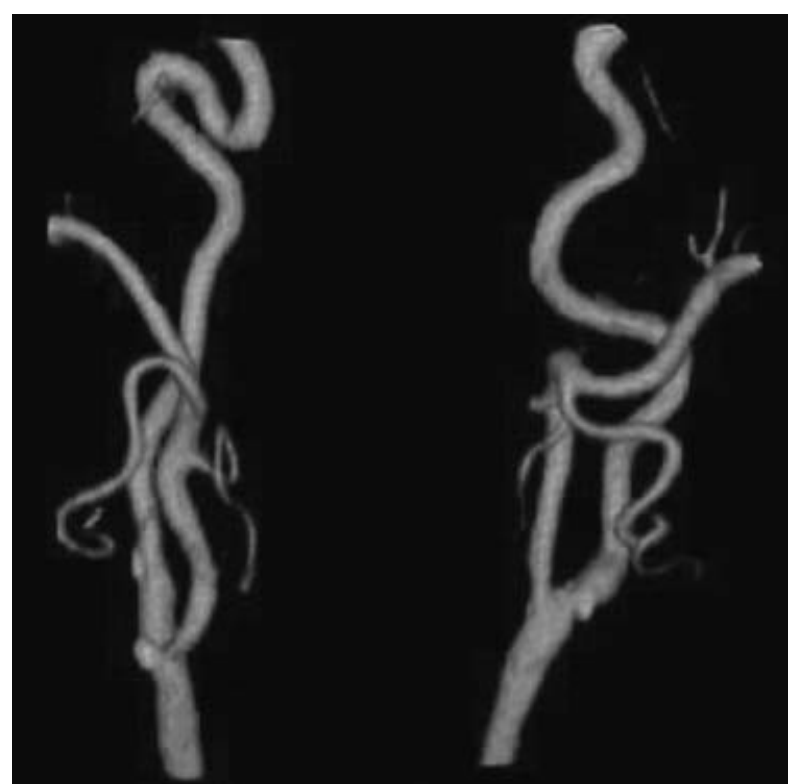

Figure 1. Vessel tortuosity.

Conclusion: Our data suggest that kinking and coiling are a frequent condition (incidence 18\%) and kinking can determine, especially in females and elderly patients, symptoms. Coiling seems not to be associated with symptomaticity. We think that Physician should consider kinking as a significant parameter in the stroke risk stratification. 


\section{P 053}

Leucoaraiosis and hypertension: are they correlated? A study by using MR

L. Saba*, L. Pascalis ${ }^{\dagger}$, G. Mallarini*

${ }^{*}$ Policlinico Universitario di Monserrato; ${ }^{\dagger}$ Ospedale San Giovanni di Dio, Cagliari, Italy

Background and objective: Cerebral white matter changes, appearing as hypoattenuated areas in CT scans and as hyperintense areas in T2-weighted MR images, are common in elderly subjects and have been termed leucoaraiosis (LA). The purpose of this study was to evaluate if there is a correlation between the presence of leucoaraiosis (identified with MR) and hypertension.

Methods and materials: We retrospectively analysed brain MR of 81 patients with age $>60 \mathrm{yr}$ ( 45 males and 36 females; age range 61-86 yr). All of the examinations were performed on a 1.5T system (Intera, Philips Medical System) equipped with an head coil. After scouts, the examination protocol included axial 3D T1weighted turbo gradient-echo images and axial T2-weighted images, which were obtained with a FLAIR sequence. We evaluated the FLAIR images of the 51 patients to rate LA extent by using the visual scale proposed by Fazekas. The we compared, by using statistical assessment, hypertension data with results about LA

Results: We observed 26 patients with LA (average Fazekas Value Scale 2.7; SD 1.1) and 31 patients with hypertension. We observed that hypertension and LA are statistically associated $(P=0.0013$; odds ratio 4.39 ).

Conclusion: The results of our study indicate that hypertension and leucoaraiosis are statistically correlated.

\section{P 054}

MDCTA in identification of cerebral aneurysms after treatment with clips

L. Saba, R. Montisci, R. Sanfilippo, G. M. Mallarini

Policlinico Universitario di Monserrato, Cagliari, Italy

Background and objective: Multi-detector row CT Angiography may be useful for screening patients with intracranial aneurysms. The purpose of this work was to evaluate MDCTA potentialities in the identification and follow-up of cerebral aneurysms after treatment with clips.

Methods and materials: We retrospectively studied the cohort population that underwent to Multi-Detector-row CT in our Department for the study and follow-up of cerebral aneurysms treated with clips. Each exam was performed by using a fourdetector-row scanner; contrast material was injected into antecubital vein and arterial phase images were obtained by using a delay time variable from 16 to 22 and by using a $5-6 \mathrm{~mL} \mathrm{~s}^{-1}$ flow rate. We used also $3 \mathrm{D}$ reconstruction technique (volume rendering) to study aneurysms two experienced neuroradiology reviewed exams.

Results: The quality of CT angiography was suboptimal in $12 \%$ of cases and in the $32 \%$ the presence of clip artifacts hampered the evaluation of the clip site. The use of three-dimensional CT angiography failed to demonstrate the presence of one aneurysm. The interobserver agreement was good $(\kappa=0.61 ; 95 \mathrm{Cl}$ $0.35-0.78)$.

Conclusion: Except for the evaluation of the site where is located the clip CT angiography showed optimal feasibility. The use of titanium clips did not affect image quality significantly, but the use of cobalt clips determine poor visualization. Sometimes very small aneurysms $(<3 \mathrm{~mm})$ can be missed.

\section{P 055}

Vascular dementia: an analysis of magnetic resonance potentialities

L. Saba*, L. Pascalis ${ }^{\dagger}$, A. Porcu*, G. Mallarini*

${ }^{*}$ Policlinico Universitario di Monserrato; ${ }^{\dagger}$ Ospedale San Giovanni di Dio, Cagliari, Italy

Background and objective: Vascular Dementia is considered one of the most common types of dementia in older adults. The term vascular dementia is reserved for indicate a progressive worsening cognitive functions and the incidence of dementia rises exponentially for patients with cerebrovascular risks such as hypertension, cardiac disease, diabetes smoking, alcoholism and hyperlipidemia. Objective of this work were: (1) to review and describe the Magnetic Resonance brain imaging characteristics of patients with vascular dementia by underlining potentialities of the new high field scanners; (2) to compare MR brain findings in patients with and without vascular dementia; and (3) to describe and review MR literature about vascular dementia.

Methods and materials: Images were acquired by using a with a 1.5-T superconducting magnet (Philips Intera). We used SE and TSE sequences, T1, T2 weighted with FLAIR sequences. Each exam was completed with of Gadolinium.

Conclusion: Magnetic resonance demonstrates optimal sensitivity, specificity in identification of the extension of lesions in patients with vascular dementia (Fig. 1). In these patients it is possible to observe white matter lesions and enlargement of central cerebrospinal fluid spaces.

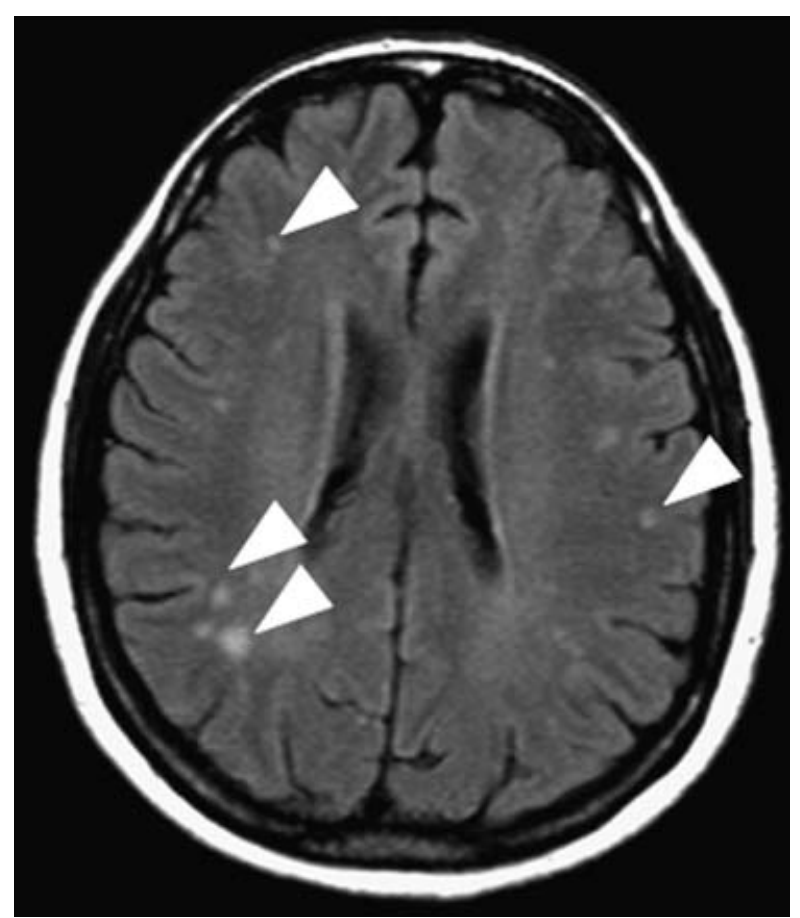

Figure 1.

\section{P 056}

\section{Near occlusion carotid artery: how to study it by using MDCTA}

L. Saba, R. Montisci, R. Sanfilippo, G. Mallarini

Policlinico Universitario di Monserrato, Cagliari, Italy

Background and objective: (1) To describe the normal anatomy of carotid artery and atherosclerotic carotid artery. (2) To review 
the concept of 'near occlusion'. (3) To review the CTA technical parameters to be used and which techniques can be employed including the visualization difference between maximum intensity projection (MIP), multi-planar reconstruction (MPR), curved planar reformation (CPR) and volume rendering (VR) for the study of 'near occlusion' carotid artery. To understand and review the relationship between near-occlusion condition and stroke.

Content organization: (1) Normal anatomy of carotid artery and atherosclerotic carotid artery. (2) Physiopathology and implications of 'near occlusion'. (3) To review the CTA technical parameters to be used and which techniques can be employed including the visualization difference between maximum intensity projection (MIP), multi-planar reconstruction (MPR), curved planar reformation (CPR) and volume rendering (VR) for the study of 'near occlusion' carotid artery.

Summary: Near-occlusion stenosis indicates a severe carotid bulb stenosis with subsequent subtle decrease in size of the distal ICA lumen. Detection and precise interpretation of nearocclusion are important to plan a proper diagnosis and treatment. The use of post processing tool is helpful in the confirmation of 'near occlusion' and in particular the CPR post-processing.

\section{P 057}

\section{Brain computed tomography characteristics in patients with vascular dementia}

L. Saba, R. Sanfilippo, R. Montisci, G. Mallarini

Policlinico Universitario di Monserrato, Cagliari, Italy

Background and objective: Vascular Dementia is a degenerative cerebrovascular disease that leads to a progressive decline in memory and cognitive functioning. This condition occurs when the blood supply carrying oxygen and nutrients to the brain is interrupted by a blocked or diseased vascular system. (1) To review and describe the computed tomography brain imaging characteristics of patients with vascular dementia underlining radiation exposure and diagnostic efficacy. (2) To understand and teach advanced imaging CT techniques as CT perfusion. (3) To compare computed tomography brain findings in patients with and without vascular dementia. (4) To learn the CT technical parameter to be used and the correct patient's position.

Methods and materials: Imaging findings in this work were obtained by using a multi-detector-row scanner. Contrast material was injected into ante-cubital vein and arterial phase images were obtained by using a delay time variable from 30 to $40 \mathrm{~s}$. We used a 3.2-mm section thickness. X-ray tube voltage was $120 \mathrm{kV}$ and tube current ranged from 240 to $300 \mathrm{mAs}$.

Conclusion: Computed Tomography can easily demonstrate white matter lesions and volumetric changes of central Cerebrospinal Fluid spaces. The use of advanced techniques as CT perfusion can dramatically improve CT sensitivity in identifying and categorizing vascular dementia.

\section{P 058}

\section{Presence of carotid artery ulcers increases symptomaticity}

L. Saba, R. Sanfilippo, R. Montisci, G. Mallarini

Policlinico Universitario di Monserrato, Cagliari, Italy
Background and objective: Stroke is a leading cause of serious, long-term disability in the United States and atherosclerosis of the extracranial carotid arteries is its major cause. The purpose of this study was to study the diagnostic efficacy of multi-detector-row spiral CT angiography (MDCTA) in the analysis of patients with carotid plaque complicated by ulceration and to evaluate is relationship with symptomaticity.

Methods and materials: We retrospectively analysed 140 patients for a total of 280 carotid arteries by using MDCTA. We evaluated the following features: degree of the stenosis (on the basis of North American Symptomatic Carotid Endarterectomy Trial Criteria : NACET) the composition of the plaque, course anomalies and particularly the presence of ulcerations. For every patients the axial images, and ANGIO MIP and 3D reconstructions were studied. In a second phase the data have been compared with the surgical results whereas the MDCTA was indicative for the surgical intervention.

Results: MDCTA found 31 ulcerations and the surgical confirmation has underlined a $92.8 \%$ sensitivity: concordance between MDCTA ad surgical results was very high We found a positive correlation between presence of ulcerations and symptomaticity $(P<0.05)$.

Conclusion: Presence of ulcerated carotid plaque is a statistically significant risk factor for ischaemic episodes.

\section{P 059}

\section{Analysis of clinical implications of air embolism in patients operated in sitting position}

I. Duda, K. Grzybowska, E. Karpel

Silesian Medical University, Department of Anesthesiology and Intensive Care, Katowice, Poland

Background and objective: One of the main risk factors during neurosurgical operations performed in sitting position is air embolism. The purpose of this study was to assess the influence of occurence of air embolism on clinical outcome in patients operated in sitting position because of pontine angle tumour.

Methods and materials: Data was collected on 244 patients, age range $15-84 \mathrm{yr}$ (average age $=51.4$ ) operated in the Department of Neurosurgery, Silesian Medical University in Katowice, Poland. All the patients were operated in sitting position in general anaesthesia TIVA using propofol and sufentanil. Air embolism was recognized on the basis of change of signal in transoesophageal Doppler sonography with a sudden drop of end-tidal carbon dioxide. Analisys was carried out to assess correlation of intrasurgical air embolism cases with the following parameters of postoperative period: death rate, hospitalization time, circulatory and/or respiratory failure rate. In the statistical analisys, U-test $(P<0.050)$ was used.

Results: Air embolism was recognised in 46 cases (19\%). Correlation between the intrasurgical air embolism with the death rate and the diagnosis of postoperative circulatory and/or respiratory failure and the hospitalization time (20.34 to 21.01 days in the group of patients without air embolism) was not proven.

Conclusion: Intrasurgical diagnosis of air embolism does not have a major influence on the postoperative course and clinical outcome. 


\section{P 060}

\section{Control over antibacterial treatment of patients with} central nervous system disease

E. A. Kondratyeva, R. V. Nazarov, A. N. Kondratyev,

E. A. Dikareva, N. A. Lesteva, S. A. Kondratyev, S. S. Lesina

Prof. Polenov Neurosurgeon Institute, St Petersburg,

Russian Federation

Background and objective: In intensive care patients with central nervous system (CNS) disease, the criteria for systemic inflammatory response syndrome (SIRS) are often unreliable as a basis for identifying the inflammatory process as they may be a sign of the diencephalo-catabolic syndrome. Diencephalo-catabolic syndrome constitutes hyperthermia of over $38^{\circ} \mathrm{C}$, tachypnea of over $20 \mathrm{~min}^{-1}$, tachycardia, and arterial hypertension. Thus, SIRS symptoms may show after antibacterial treatment even if there is no infection or inflammation. We suggest a more precise method to control over antibacterial treatment in patients with CNS disease and to determine the best duration of antibacterial treatment - a procalcitonin test makes the control over antibacterial treatment of patients with CNS disease more precise; reduces the number of complications related to unnecessarily long antibacterial treatment.

Methods and materials: After a course of antibacterial treatment, on the day following the last antibacterial injection a procalcitonin test is taken, i.e. the level of procalcitonin in the patient's serum is determined by a semiquantitative method on a disposable BRAHMS PCT-Q system. The procalcitonin level is determined against a colour scale. Procalcitonin level of over $0.5 \mathrm{ng} \mathrm{mL}^{-1}$ is a sign of infection and an indication that it is necessary to continue antibacterial treatment. If the test result is negative and there are no point of infection, no antibacterial treatment is administered even if there are SIRS symptoms.

Results: The method was developed and clinically tested on 30 patients in the intensive care department.

Conclusion: Procalcitonin test makes it possible to determine how effective the administered antibacterial treatment is, as well as to determine its best duration, thus reducing the number of complications as a result of antibacterial treatment and medical costs.

\section{P 061}

\section{Amantadine in vegetative state and minimal conscious state}

E. A. Kondratieva*, R. V. Nazarov*, A. V. Schegolev ${ }^{\dagger}$, L. M. Zenziper*

${ }^{*}$ Russian Neurosurgical Institute, Department of Intensive Care; ${ }^{\dagger}$ Military Medical Academy, St Petersburg, Russian Federation

Background and objective: The underlying idea here is that in a coma the reserves of endogenic dophamine become insufficient, which results in bradykinesia and low cognitive level. Further recovery of consciousness and motor activity depends on how fast the concentration of dophamine is restored. The aim was to study the effectiveness of amantadine sulphate (PK-Merz) for the recovery of consciousness during rehabilitation in patients with brain damage.

Methods and materials: The Section of Neural Intensive Care and Neural Anesthesiologists of the Federation of Anesthesiologistsof Russia decided to conduct a prospective, non-randomized, uncontrolled open 'before and after' study. Three medical centres took part in the study, with a total of 25 patients. All patients that met the criteria to be included in the study underwent clinical testing, EEG monitoring (24-h monitoring), and neurovisualization (CT/MRI). The level of consciousness was assessed according to the Glasgow Consciousness Scale. The outcome was assessed according to the Glasgow Outcome Scale. During the first three days $1000 \mathrm{~mL}(400 \mathrm{mg})$ were drip-fed intravenously. Starting on the fourth day, the patients were administered oral PK-Merz $-400 \mathrm{mg}$. On the 11th-28th day the daily dose was $200 \mathrm{mg}$. The statistical data analysis was conducted in NCSS and PASS 2004 software (by J. Hintze).

Results: The outcome according to the Glasgow Outcome Scale became higher during treatment (Graph 2), and by the 7th day a reliable difference $(P<0.001)$ from the measure before treatment, 3 (IQR 3; 4) to 3 (IQR 3; 3) before treatment, was observed. EEG monitoring showed the tendency of cyclicity and sleep structure during the PK-Merz treatment. Brain CT/MRI showed that in all patients there was no uncorrected occlusive hydrocephaly, a cerebral process with a mass effect during the treatment.

Conclusion: During the PK-Merz treatment, the improvement of neurophysiological indicators and outcome was observed.

\section{P 062}

\section{Anticoagulation in a neurosurgical patient with heparin-induced thrombocytopenia (HIT type II) with Argatroban and Fondaparinux}

T. Kerz, H. S. Schinzel

Mainz, Germany

Background and objective: Heparin-induced thrombocytopenia has an incidence of $0.1-1 \%$ with low-molecular weight heparins $(\mathrm{LMWH})$ and $0.1-3 \%$ with unfractioned heparins (UFH). Characteristic clinical signs are a drop in platelet count of $>50 \%$ associated with new thromboembolic complications during heparin therapy in up to $70 \%$ of cases. We describe a case of a HIT II in a neurosurgical patient which was successfully managed with the initial use of argatroban and, later, fondaparinux.

Case report: We report on a patient with a subarachnoidal haemorrhage. After clipping of the aneurysm, she was placed on prophylaxis for venous thromboembolism with Dalteparin, 5.000 IE subcutaneously qd on postoperative day 1 . On day 6 , a chest CT showed extensive pulmonary embolism. Due to major right ventricular dysfunction, a surgical embolectomy was carried out. Therapeutic anticoagulation was achieved by intravenous unfractionated heparin (UFH) administration. Platelet counts on the day of admission were $370 \mathrm{~nL}^{-1}, 194 \mathrm{~nL}^{-1}$ on the day of pulmonary embolism, and fell to $70 \mathrm{~nL}^{-1}$ on day 11 when HIT II was suspected (Fig. 1). Heparin was replaced by argatroban, and platelet counts were back to $159 \mathrm{~nL}^{-1}$ on day 15. ELISA testing

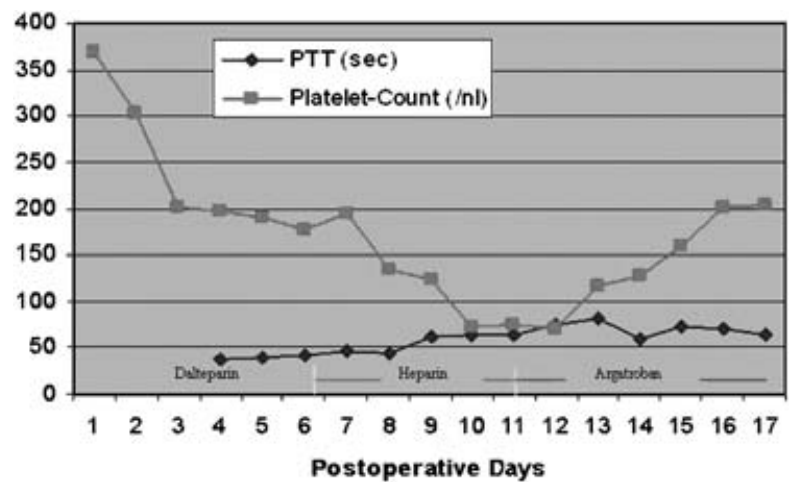

Figure 1. 
for heparin antibodies was negative, as was a complete thrombophilia study. Argatroban was given by continuous infusion for 7 days when it was replaced by Fondaparinux $2.5 \mathrm{mg}$ bid. Cranial CT-scans showed no signs of intracranial bleeding during anticoagulation therapy.

Conclusion: The patient presented a therapeutic dilemma as she had undergone a major neurosurgical operation but needed therapeutic anticoagulation while HIT II was suspected because of the constant fall of the platelet counts and a newly developed pulmonary embolism. Argatroban and Fondaparinux were both safe in this neurosurgical patient and may have further potential in such a setting. Return of platelet count to normal after cessation of heparin confirmed the diagnosis of HIT II.

\section{P 063}

Effects of $15 \%$ Mannitol, $10 \% \mathrm{NaCl}$ and HyperHAES on the ICP, oxygen delivery and cerebral oxygenation in patients with the intracranial haemorrhage

S. Petrikov, V. Krylov, A. Solodov

Sklifosovsky Research Institute, Department of Neurosurgical ICU, Moscow, Russian Federation

Background and objective: The 'ideal' hypertonic solution for intracranial hypertension treatment is still under discussion. We evaluated effects of hypertonic solutions on the intracranial pressure (ICP), oxygen delivery $\left(\mathrm{DO}_{2}\right)$ and cerebral $\mathrm{O}_{2}$ extraction ratio $\left(\mathrm{CO}_{2} \mathrm{ER}\right)$ in patients with the intracranial haemorrhage $(\mathrm{IH})$.

Methods and materials: In all, 12 patients with IH and GCS 4-9 enrolled in the study. Patients had invasive monitoring of ICP, $\mathrm{SjO}_{2}$ and central haemodynamycs (PICCO plus). ICP higher than 20 torr was an indication for treatment. We used $15 \%$ Mannitol $400 \mathrm{~mL}(n=13), 10 \% \mathrm{NaCl} 200 \mathrm{~mL}(n=7)$ and HyperHAES $(7.2 \%$ $\mathrm{NaCl}$ in $6 \% \mathrm{HES} 200 / 0.5) 250 \mathrm{~mL}(n=13)$. ICP, CPP, $\mathrm{DO}_{2}, \mathrm{CO}_{2} \mathrm{ER}$ were recorded before infusion and 5, 30, $120 \mathrm{~min}$ after it.

Results: Mannitol decreased ICP from (mean \pm SD) $32 \pm 8$ to $16 \pm 7$ torr $(P<0.05)$. After 30 and $120 \mathrm{~min}$, ICP was $17 \pm 9$ $(P<0,05)$ and $24 \pm 11$ ltorr. The $10 \% \mathrm{NaCl}$ decreased ICP from $34 \pm 9$ to $14 \pm 4$ torr $(P<0.05)$. After 30 and $120 \mathrm{~min}$, ICP was $14 \pm 7(P<0.05)$ and $24 \pm 7$ torr. HyperHAES decreased ICP from $29 \pm 6$ to $16 \pm 8$ torr $(P<0.05)$. After 30 and $120 \mathrm{~min}$ ICP was $11 \pm 8(P<0.05)$ and $15 \pm 9$ torr $(P<0.05)$. Mannitol increased CPP from $76 \pm 18$ to $96 \pm 19$ torr $(P<0.05)$. After 30 and $120 \mathrm{~min}$, CPP was $98 \pm 21(P<0.05)$ and $89 \pm 19$ torr. The $10 \% \mathrm{NaCl}$ did not change CPP significantly. HyperHAES increased CPP from $70 \pm 16$ to $92 \pm 16$ torr $(P<0.05)$. After 30 and $120 \mathrm{~min}$, CPP was $89 \pm 15(P<0.05)$ and $86 \pm 16$ torr $(P<0.05)$. Mannitol and $10 \%$ $\mathrm{NaCl}$ did not influence $\mathrm{DO}_{2}$ and $\mathrm{CO}_{2} \mathrm{ER}$ significantly. HyperHAES increased $\mathrm{DO}_{2}$ from $436 \pm 112$ to $608 \pm 168 \mathrm{~mL} \mathrm{~min}^{-1} \mathrm{~m}^{-2}$ 30 min after infusion $(P<0.05)$ and decreased $\mathrm{CO}_{2} \mathrm{ER}$ from $0.3 \pm 0.1$ to $0.2 \pm 0.130$ and 120 min after infusion $(P<0.05)$.

Conclusion: Infusion of $15 \%$ Mannitol, $10 \% \mathrm{NaCl}$ and HyperHAES is an effective method of ICP control. HyperHAES has the most prolong effect on ICP, markedly increases $\mathrm{CPP}, \mathrm{DO}_{2}$ and decreases cerebral $\mathrm{O}_{2} \mathrm{ER}$.

\section{P 064}

Comparison between $0.9 \%$ sodium chloride and $5 \%$ dextrose water solutions in symptoms relief in acute migraine attacks: a pilot single-blinded clinical trial Y. Jahangiri Noudeh ${ }^{\star}$, F. Sina ${ }^{\dagger}$, M. Shabani

*Iran University of Medical Sciences, Medical Students Research Committee; 'Iran University of Medical Sciences, Rasoul-eAkram Medical Center; "Iran University of Medical Sciences, Faculty of Medicine, Department of Biochemistry, Tehran, Iran
Background: Dehydration has been reported in acute migraine attacks, and fluid replacement therapy could relieve its symptoms. However, administered fluid composition may be effective in headache relief process in acute migraine attacks.

Objectives: To compare between sodium chloride and dextrose solutions in symptoms relief of acute migraine attacks.

Methods and materials: We selected eight patients with known chronic migraine-type headaches referred to Emergency Department for acute attack of headache, and allocated them in two groups. The first group $(N=4,2$ males and 2 females, mean age $=29.0 \mathrm{yr} ; \mathrm{SD}=5.3 \mathrm{yr}$, mean headache duration $=11.4 \mathrm{yr}$; $\mathrm{SD}=5.0 \mathrm{yr}$ ) administered with $500 \mathrm{~mL}$ of $0.9 \%$ sodium chloride solution infusion during $15 \mathrm{~min}$. Second group $(N=4$, all males, mean age $=27.2 \mathrm{yr} ; \mathrm{SD}=7.7 \mathrm{yr}$, mean headache duratio$n=7.2 \mathrm{yr} ; \mathrm{SD}=6.1 \mathrm{yr}$ ) received $500 \mathrm{~mL}$ of $5 \%$ dextrose solution infusion in $15 \mathrm{~min}$. The patients were unaware of administered fluid type and they were monitored for their attacks recur for up to $30 \mathrm{~min}$ after infusion completed.

Results: The reduction in pain severity in all patients in Saline group was considerable and there was no need to use analgesics after about $30 \mathrm{~min}$ of infusion completion, while dextrose solution group need to take analgesic and anti-emetic therapy after $5 \mathrm{~min}$ of infusion completed. This was not statistically significant $(P=0.143$, Fischer's exact test). On the other hand, nausea and vomiting were the first symptoms relieved during Saline infusion, however, there was no improvement in nausea and vomiting condition in second group.

Conclusions: Although not statistically significant, our results could open new horizons in abortive therapy profile of migraine attacks and gleams to make new explanation to headaches pathophysiology. It seems that more clinical studies with greater sample sizes are yet needed in this era.

\section{P 065}

'Pressure inside the neuroendoscope' is more reliable than epidural intracranial pressure during neuroendoscopic procedures

R. Valero*, L. Salvador ${ }^{\dagger}$, L. Caral ${ }^{\dagger}$, E. Ferrer ${ }^{\dagger}$, E. Carrero ${ }^{\dagger}$, J. Tercero ${ }^{\dagger}$, N. Fàbregas ${ }^{\dagger}$

*Hospital Clinic de Barcelona, Department of Anesthesiology; †Hospital Clínic, Barcelona, Spain

Background and objective: Intracranial pressure (ICP) recording during neuroendoscopic procedures is mandatory in order to detect CBF abrupt changes [1,2]. Pressure inside the endoscope (PIN) by connecting a fluid-filled catheter from the irrigation lumen of the neuroendoscope to a pressure transducer is an easy way to control ICP. Intraoperative epidural ICP recording (EICP) can be thought as a safe and more rigorous alternative to PIN. Our aim was to compare both EICP and PIN during neuroendoscopies.

Methods and materials: After Ethics Committees' approval, patients scheduled for a neuroendoscopic procedure were prospectively studied. Patient characteristics, type of lesion, length of procedure, intraoperative and postoperative complications were recorded. Epidural ICP values (Neurodur epidural, Rehau. Germany), from immediately after the burr hole until the end of the neuronavigation time, and PIN values during neuronavigation time were continuously recorded at $2 \mathrm{~Hz}$. Brand-Altman test was used to assess agreement between pressures.

Results and discussions: In all, 17 consecutive patients were included: 8 males $/ 9$ females, age $49.2 \pm 17.3 \mathrm{yr}$. Secondary hydrocephalus was the most frequent diagnosis. Mean neuronavigation time was $22.33 \pm 14.5 \mathrm{~min}$. Correlation between EICPPIN was strong in $15 / 17$ patients (range $R 2=0.57-0.93$ ). The 


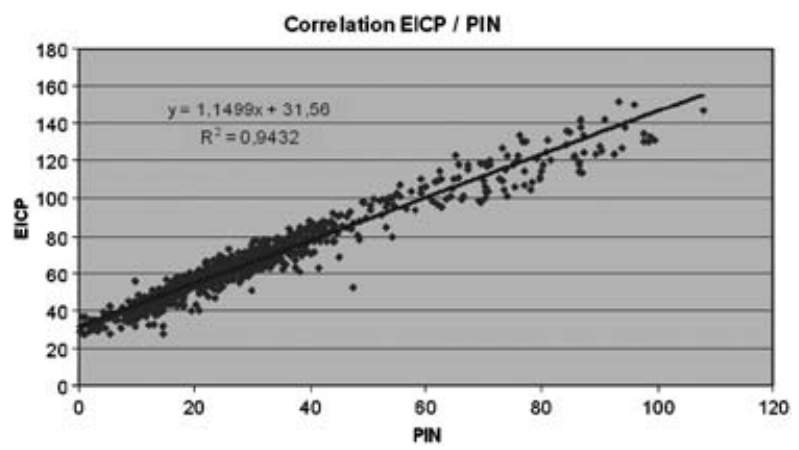

Figure 1. Correlation EICP/PIN in Correlation EICP/PIN in case 4.

mean differences between EICP-PIN were $20 \pm 24 \mathrm{mmHg}$. EICP were systematically higher than PIN values in 15/17 patients. Using Bland-Altman analysis, PIN-EICP methods showed a good agreement in six patients. However, in 11 patients discrepancies were greater with higher ICP values. No intraoperative or postoperative complication was related to monitoring.

Conclusions: A mild correlation was found between absolute EICP and PIN, but EICP values were systematically higher than PIN values, this drift being more marked at higher pressures.

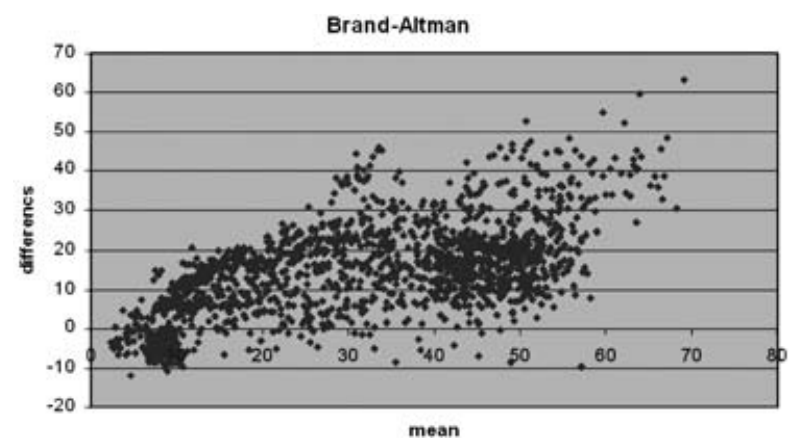

Figure 2. Brand Altman test for EICP and PIN in case Brand Altman test for EICP and PIN in case 7.

These intraoperative results correlate with previous studies outside the operating room comparing epidural and intraventricular pressures $[3,4]$.

\author{
References \\ 1. J Neurosurg Anesthesiol 2000; 12: 21-28. \\ 2. J Neurosurg Anesthesiol 2001; 13: 152-157. \\ 3. Int Care Med 1995; 21: 850. \\ 4. J Neurosurg 2007; 106: 548.
}

\section{NEUROCOGNITIVE DYSFUNCTION/DELIRIUM}

\section{P 066}

\section{Recovery and cognitive function after total intravenous anaesthesia with or without dexmedetomidine for lumbar discectomy}

\section{G. A. Gurbet, H. Bilgin, B. Ozcan}

Uludag University, Medical Faculty, Department of Anesthesiology and Reanimation, UUTF Anesteziyoloji ve Reanimasyon AD, Bursa, Turkey

Background and objective: We evaluated awakening, recovery and restoration of cognitive functions, and cost of remifentanilpropofol (total intravenous anaesthesia-TIVA) or remifentanilpropofol + dexmedetomidine (TIVA+D) anaesthesia in patients undergoing lumbar disc surgery lasting 60-120 min.

Methods and materials: After approval by the local ethical committe and after obtaining written informed consent, 40 patients with ASA I-II were enrolled in this randomized, doubleblinded, prospective controlled study. Patients were allocated to either the TIVA $(n=20)$ or the TIVA+D $(n=20)$ group. Anaesthesia induction was obtained with a bolus dose of propofol $\left(1.5-2 \mathrm{mg} \mathrm{kg}^{-1}\right)$, maintenance was achieved with a propofol and remifentanil infusion throughout the procedure. The TIVA+D group received remifentanil and propofol as TIVA group, plus dexmedetomidine infusion $0.5 \mu \mathrm{g} \mathrm{kg}^{-1} \mathrm{~min}^{-1}$. The infusion rate of propofol and remifentanil was changed according to bispectral index (BIS) ratio and haemodynamics. Muscle relaxation was achieved with rocuronium. Intraoperative haemodynamics, recovery, PACU stay, direct drug acquisition and postoperative pain treatment were documented. The Mini-Mental State (MMS) test was used to assess cognitive functions preoperatively and postoperatively $2 \mathrm{~h}, 24 \mathrm{~h}, 1$ week and 1 month.

Results: There were no significant differences in immediate recovery characters, Alderete's Scores, time of PACU stay, MMS scores, and direct drug acquisition between the groups.
Intraoperative haemodynamics were better preserved and remifentanil requirement was significantly less in patients receiving TIVA+D. Also, significantly less patients suffered postoperative pain in this group.

Conclusion: Patients undergoing lumbar discectomy showed no significant differences in awakening, recovery and restoration of cognitive functions after TIVA or TIVA+D anaesthesia. In addition, our data suggest that the addition of dexmedetomidine in propofol-remifentanil anaesthesia has no spesific effect on return of cognitive functions.

\section{P 067}

The role of global cerebral hypoperfusion in the development of postoperative cognitive dysfunction

S. M. Moritz, A. M Arlt, V. S. Völkel, R. L. Rupprecht,

H. J. Hobbhahn

University of Regensburg, Department of Anesthesiology,

Regensburg, Germany

Background and objective: Postoperative cognitive dysfunction (POCD) is a common problem following cardiac surgery. However, the aetiology of POCD is still unknown. The present study was designed to evaluate whether global cerebral hypoperfusion plays a major role in the development of POCD in patients undergoing coronary artery bypass grafting (CABG). Methods and materials: In 82 consecutive patients (32 offpump; 50 on-pump) undergoing elective CABG, jugular bulb venous oxygen saturation $\left(\mathrm{SjO}_{2}\right)$, the arterial-jugular venous lactate content difference (AJDL) and relative changes of regional cerebral oxygen saturation measured by near infra-red spectroscopy ( $\Delta \% \mathrm{NIRS})$ were recorded preoperatively, at five predefined time points during operation and $1 \mathrm{~h}$ after arrival at the ICU. Pathological values were defined as follows: $\mathrm{SjO}_{2}<55 \%$, 
AJDL $>0.16 \mathrm{mmol} \mathrm{L}^{-1}$ and $\Delta \% \mathrm{NIRS}>20 \%$. In each patient, the number of pathological values for each parameter was recorded. To detect POCD all patients completed a battery of neurocognitive tests before and 7 days after CABG. Cognitive dysfunction was defined as a $>25 \%$ decrease in $20 \%$ of the tests.

Results: Postoperative cognitive dysfunction occurred in 36 patients $(43.9 \%)$. Pathological values for $\mathrm{SjO}_{2}$ were found in 49 patients $(60 \%)$, for AJDL in 20 patients $(24 \%)$ and for $\Delta \%$ NIRS in 14 patients (17\%). The number of $\mathrm{SjO}_{2}$ desaturations was significantly higher in patients with POCD $(P<0.05)$, while there was no difference for AJDL and $\Delta \%$ NIRS. In a logistic regression analysis age $>60 \mathrm{yr}$ (odds ratio $2.8(1.09-7.48) ; P<0.05)$ was an independent predictor of POCD, but not the number of pathological values for $\mathrm{SjO}_{2}$, AJDL and $\Delta \% \mathrm{NIRS}$.

Conclusion: Neither jugular bulb venous monitoring nor near infra-red spectroscopy could predict the occurrence of POCD. Thus, according to our data, global cerebral hypoperfusion does not seem to play a major role for the development of postoperative cognitive dysfunction. Age $>60 \mathrm{yr}$ was the only independent predictor of POCD.

\section{PROGNOSIS AND OUTCOME PREDICTION}

\section{P 068}

Recovery of consciousness from a vegetative state: a functional or morphological phenomenon?

E. A. Kondratyeva, A. N. Kondratyev

Prof. Polenov Neurosurgeon Institute, St Petersburg, Russian Federation

Background and objective: The study was based on the hypothesis that in some patients the vegetative state (vs) is a result of the establishment of a stable pathological system that limits the functional activity of the brain. The identification of such a system may make it possible to make further prognoses on the probability of the recovery of consciousness.

Aim: The purpose of the present study was to identify the role of the functional states of the brain that determine the recovery of consciousness or the lack thereof in patients in a vegetative state.

Methods and materials: The study is based on the results of the examination, treatment and observation of 34 patients in vs. Patients in a vegetative state show three EEG patterns: the low frequency EEG pattern, the generalized slow wave pattern and disorganized polymorphic EEG pattern. After the EEG, the benzodiazepine test was taken intravenous $\left(0.04 \mathrm{mg} \mathrm{kg}^{-1}\right.$ of midazolam). Test results were considered positive if after the intravenous benzodiazepine injection, the EEG pattern changed.

Results: The test result was considered negative if after the maximum dose of benzodiazepines was reached, the EEG pattern did not change (22 patients). All of the patients that showed positive test results (12 patients) recovered their consciousness; therefore, the test has value for prognosis. The analysis of the treatment outcome in patients who demonstrated positive benzodiazepine test results according to the Glasgow Consciousness Scale was undertaken 6 months after the recovery of consciousness. The patients who did not demonstrate EEG change remained in the vegetative state for the following $2 \mathrm{yr}$ under observation (22 patients).

Conclusion: We suppose that the emergence of bioelectric brain activity close to normal after the injection of benzodiazepines demonstrates the presence of a functional component in the structure of the vegetative stat.

\section{P 069}

Recurrence rate in ischaemic and heorrhagic strokes

S. Shaafi

Tabriz University of Medical Science, Department of Neurology, Neurology Ward - Razi Hospital, Tabriz, Iran

Background and objective: Stroke, positioning in the third place of the most fatal diseases produces instabilities and profound socio-economical society. These problems are intensified in recurrent stroke.

Aim: Aim of study to find out stroke recurrence and mortality and scrutinize the relevant risk factors.

Methods and materials: According to the previous reports the overall $17 \%$ rate of recurrence in stroke with the replacement of $\mathrm{p}, \mathrm{q}, \mathrm{d}$ and $\mathrm{z}$ in the formula $[P=17 \%, \mathrm{q}=0.83, \mathrm{z}=1.96$ and $\mathrm{d}=7]$ we selected 300 patients (34-85yr old) who had been admitted in the neurology ward of Razi hospital for their first stroke (proven stroke) and after discharge of them they followed up monthly for $5 \mathrm{yr}$ over a period of 60 months. Selection was randomly (simple randomized sampling) (150 ischaemic and 150 haemorrhagic cases). In the cases that new signs or symptoms in favor of a new attack were detected and if clinically warranted brain CT scan or MRI were used for paraclinically approve the recurrence of stroke.

Results: The cumulative risk of occurrence among selected cases encompassing $56 \%$ male and $44 \%$ female was $32 \%$ to which males and females contributed $12 \%$ and $20 \%$, respectively $(67.5 \%$ female vs. $32.5 \%$ male, $P$ value $=0.00)$. For initial stroke HTN (78\%), smoking (43\%) and HLP (26\%) were the most probable risk factors attributable to the event. In recurrent stroke HTN (28\%), HLP (11\%), smoking (9\%) and IHD (9\%) were the most important factors. Mean time of the recurrence after the initial stroke was $5.3 \pm 3.4$ months.

Conclusion: Because of the fact that the mortality rates and disabilities with recurrent stroke are usually more vigorous than initial events, secondary prevention of stroke must be considered seriously. This study highlights the importance of strict hypertension, ischaemic heart disease and hyperlipidemia control and cease of smoking.

\section{P 070}

Initial GCS is an unreliable predictor of outcome in patients with severe head injury treated (sTBI) by an ICP targeted therapy. A prospective study

L.-O. D. Koskinen*, M. Olivecrona ${ }^{\dagger}$,

M. Rodling-Wahlström", S. Naredi"

*Umea University Hospital, Neurokirurgen, NUS, Department of Neurosurgery; 'Departmet of Neurosurgery; "Department of Anaesthesiology and Intensive Care, UMEA, Sweden

Background and objective: Previous studies have shown a correlation between initial GCS and outcome after sTBI. This correlation has been used to prognosticate outcome assessed as GOS. The aim was to investigate the value of GCS in prognostication of outcome in STBI.

Methods and materials: Patients with sTBI admitted 2002-2005. Inclusion criteria: GCS at intubation and sedation $\leqslant 8$, age $15-70 \mathrm{yr}$, first recorded $\mathrm{CPP} \geqslant 10 \mathrm{mmHg}$, and arrival 
within $24 \mathrm{~h}$. Exclusion criteria: pregnant/lactating woman, penetrating injury. Protocol guided treatment based on the Lund concept was used. Independent staff evaluated the GOS at 3 and 6 months. Contingency analysis was used for evaluation of overall effects and ROC curve analysis was employed to evaluate the value of GCS in the prognostication of the dicotomized GOS (unfavourable GOS 1-3 and favourable GOS 4-5) outcome at 3 and 6 months.

Results: In all, 48 patients, mean age $35.5 \mathrm{yr}$, were included, no patient were lost to follow-up. Median GCS at intubation and sedation was $6(3-8)$. Contingency analysis revealed no overall effects between the initial GCS and GOS at 3 months $(P=0.533)$ and 6 months $(P=0.269)$. Using the 3 months dicotomized GOS and the GCS value with highest accuracy $(G C S \leqslant 4)$ ROC analysis showed a sensitivity of $52.2 \%$ and specificity of $80.0 \%$. The positive predictive value was $70.6 \%$ and negative prognostic value $64.5 \%$. Positive likelihood ratio was 2.61 and negative likelihood ratio 0.60 . Similar results were found using the 6 months outcome. If mortality prognostication is the ultimate goal the corresponding values are $30.4 \%, 84 \%, 63.6 \%, 56.8 \%$, $1.90 \%$ and $0.83 \%$, respectively.

Conclusion: In patients treated with an ICP targeted therapy, the prognostic value of initial GCS is insufficient to securely prognosticate the outcome. Based on our findings no absolute treatment decisions can be based on this variable.

P 071

Recurrence rate of haemorrhagic and ischaemic
strokes

S. Shaafi

Tabriz University of Medical Science, Department of Neurology, Neurology Ward - Razi Hospital, Tabriz, Iran

Background and objective: Stroke, positioning in the third place of the most fatal diseases produces instabilities and profound socio-economic as well as psychological problems for both individuals and society. These problems are intensified in recurrent stroke. We carried out a research to find out of stroke recurrence and mortality and scrutinize the relevant risk factors.

Methods and materials: We selected 300 patients (34-85yr old) who had been admitted in the neurology ward for their first stroke (proven stroke) and after discharge of them they followed up monthly for $5 \mathrm{yr}$ over a period of 60 months from March 2000 to March 2005.

Selection was randomly (simple randomized sampling) (150 ischaemic and 150 haemorrhagic cases).

Results: The cumulative risk of occurrence among selected cases encompassing $56 \%$ male and $44 \%$ female was $32 \%$ to which males and females contributed $12 \%$ and $20 \%$, respectively $(67.5 \%$ female vs. $32.5 \%$ male, $P$ value $=0.00)$. For initial stroke HTN (78\%), smoking (43\%) and HLP (26\%) were the most probable risk factors attributable to the event. In recurrent stroke HTN (28\%), HLP (11\%), smoking (9\%) and IHD (9\%) were the most important factors. Mean time of the recurrence after the initial stroke was $5.3 \pm 3.4$ months. In all, $21 \%$ of the patients with positive ischaemic heart disease developed ischaemic stroke $(95 \%$ ischaemic stroke, $5 \%$ haemorrhagic stroke, $P$ value $=0.05$ )

Conclusion: Because of the fact that the mortality rates and disabilities with recurrent stroke are usually more vigorous than initial events, secondary prevention of stroke must be considered seriously. This study highlights the importance of strict hypertension, ischaemic heart disease and hyperlipidaemia control and cease of smoking.

\section{P 072}

\section{Anisocoria and loss of pupillary reflex before $\mathrm{CT}$ in severe head injury: a series of 1774 cases}

L. Levi

Rambam Medical Center, Bat Galim, Haifa, Israel

Background and objective: All comatose cases with acute head trauma should have a CT scan. Previous assessment thought that anisocoria and loss of pupillary reaction to light of the mydriatic pupil is highly indicative of unilateral mass lesion.

Methods and materials: We screened our neurotrauma database of 1996-2005 with 1774 severe head-injured patients and found anisocoria with unilateral papillary reaction loss in 175 patients - before CT scan was done.

Results: The CT examination yielded 567 patients with mass lesion needing surgical evacuation (31.4\% of the patients). If we take out the bilateral mydriatic patients 199 (11.2\%) the incidence of evacuated haematoma is $42.3 \%$ of the patients with anisocoria compared with $28.2 \%$ of the patients with both pupils equal and reacting at the time of CT. This Odds ratio of 1.86 is barely significant.

Conclusion: We conclude that relying on this fairly common finding before CT with such low sensitivity (42.3\%) and specificity $(28.2 \%)$ is almost good as guessing. Further study of other confounders and predicting variables is underway to continue answering the need for CT in patients you already decided to transfer to neurotrauma based on their clinical status.

\section{P 072 A}

\section{Extra cerebral organ dysfunction in a mixed} neurosurgical intensive care patient population

L. Engborg ${ }^{*}$, A. Theodorsson ${ }^{\dagger}$, J. Hillman ${ }^{\dagger}$,

A. Samuelsson*, I. Steinvall", F. Sjöberg ${ }^{\star}$,

Departments of *Intensive, ${ }^{\dagger}$ Neurosurgery, and Plastic Surgery,

'Linköping University Hospital, Linköping, Sweden

Background and objective: A pilot study to describe the incidences of extra-cerebral organ dysfunction and to grade ICU-acquired complications in a neurosurgical ICU population using a newly developed web-based ICU-scoring programme. Methods and materials: Neurosurgical intensive care unit (NIVA) Department of Neurosurgery, Linköping University Hospital, Sweden. A regional referral centre for the southeastern region of Sweden comprising a population of 1.4 million.

Patients: In all, 87 patients were registered according to the Swedish ICU registry inclusion criteria. We identified mainly two neurosurgical diagnoses SAH (aneurysmal subarachnoid haemorrhage) and trauma patients that constituted about $50 \%$ of the cohort.

Interventions: None.

Results: APACHE II score was 11.4 (median 16; 2-28 (range)). Organ failures as defined by a score of $>3$ in SOFA (Sequential Organ Failure Assessment) were: Respiratory failure in 35 pats (37\%) comprising 26 pats (30\%SOFAresp 3p) and 4 pats (5\%SOFAresp $4 p)$. Cardiovascular failure in 28 pats $(32 \%)$. Combined cardiopulmonary failure had 30 pats were 18 pats had $\mathrm{SAH}$ and 6 pats had TBI. The other extra-cerebral organ systems were not significantly affected. Respiratory failure defined by the lung injury score (LIS) showed that 48 pats $(55 \%)$ had mild to moderate lung injury and 13 pats $(15 \%)$ had ARDS/Severe respiratory failure. Respiratory failure defined by Euro-american consensus conference on ARDS showed that 31 pats $(35 \%)$ had ALI and 35 pats $(40 \%)$ had ARDS. SIRS was registered in 26 pats $(30 \%)$, Sepsis in 26 pats $(30 \%)$, Severe Sepsis in 10 pats $(11 \%)$ and Septic shock in 3 pats (4\%). Ventilator-associated pneumonia VAP as defined by Clinical 
Pneumonia Infection Score (CPIS) was found in 6 pats $(6 \%)$. Mean New Injury Severity Score (NISS) in the trauma patients was 30 points (range 14-68).

Conclusion: The database tool developed worked smoothly in the everyday setting and recording was feasible. The data show that there are a significant number of extra-cerebral complications in this cohort. The finding underlines the need for good follow-up tools that may be used in the development of care plans in order to improve outcome. The registry can also be used in audits and other quality issues.

\section{P 073}

Changing practices in providing perioperative analgesia for craniotomy - a national survey

H. P. Krovvidi, R. Balakrishnan ${ }^{\dagger}$, N. Huggins ${ }^{\dagger}$, M. Smith ${ }^{\dagger}$

'University of Birmingham, Birmingham, UK

Background and objective: Previous two surveys of neuroanaesthetists across Great Britain and Ireland showed intramuscular Codeine Phosphate was commonest postoperative analgesia after intracranial surgery. We intended to repeat the survey due to several changes in neuroanaesthesia practices.

Methods and materials: A postal questionnaire was sent to all practicing consultant neuroanaesthetists $(n=175)$ in Great Britain and Ireland through the local Neuroanaesthesia Society of Great Britain and Ireland (NASGBI) linkmen.

Results: The response rate was $57.71 \%(n=101)$. Majority of anaesthetists $86 \%(n=87)$ used remifentanil as an agent of choice for providing intraopertive analgesia during the intracranial surgeries. $31.6 \%(n=32)$ of anaesthetists responded saying that they had changed choice of either intraoperative or postoperative analgesic agent of choice in recent times. Patient controlled analgesia was the preferred method of choice of postoperative analgesia for $44.5 \%(n=45)$ of responders. For immediate postoperative analgesia in recovery room after craniotomy, morphine was the agent of choice either in the form intravenous boluses or PCA. Intravenous paracetomol and intramuscular codeine phosphate was second and third line agents for analgesia, respectively. Oral codeine phosphate or dihydrocodiene and oral paracetomol were analgesic agents used for craniotomies in wards postoperatively. In all, $32.6 \%$ $(n=33)$ of anaesthetists used NSAIDS for either intraoperative or postoperative analgesia.

Conclusions: Introduction of remifenatnil, intravenous paracetamol and COX-2 NSAIDs had changed the practice of providing pain relief during and after craniotomy in the United Kingdom. Morphine sulphate (intravenous) had replaced codeine phosphate (intramuscular) to provide immediate postoperative analgesia compared to previous two surveys. Codeine remained a popular choice to provide the ward-based analgesia after craniotomy. Patient controlled analgesia (PCA) with morphine is prescribed by more neuroanaesthestists compared to previous surveys.

\section{P 074}

\section{Alleviate allodynia and hyperalgesia by silencing of Nav1.8 with siRNA in bone cancer pain rats}

X. L. H. Xu, L. Yanhong, J. Yuge, Z. Hong

PLA General Hospital, Department of Anesthesiology, Beijing, China

Background and objective: Nav1.8 is a tetrodotoxin resistant sodium channel and its restricted expression to the peripheral sensory neurons suggest that blocking this channel might have therapeutic potential in various pain states. The aim was to investigate the antinociceptive effect of siRNA targeting Nav1.8 in a rat model of bone cancer pain.
Methods and materials: (1) Female SD rats recieved intra-tibial injection of 5104 syngenetic Walker256 mammary gland carcinoma cells. Mechanical allodynia and thermal hyperalgesia were tested after cell injection. (2) Three pairs of siRNA targeting Nav1.8 by in vitro transcription were synthesized. siRNA were transfected into primary cultured dorsal root ganglion neurons and mRNA expression of Nav1.8 was determined with RT-PCR and Q-PCR $48 \mathrm{~h}$ after the transfecion. (3) We synthesized siRNA chemically according to the sequence that can most effectively attenuation of Nav1.8 mRNA in vitro and $3^{\prime}$ terminal of siRNA was methyl modified (M-siRNA). M-siRNA, negative control RNA and normal saline were delivered as repeated daily bolus doses (25-100 $\mu \mathrm{g}$, for 7 days) via implanted intrathecal catheter to the lumbar spinal cord of bone cancer rats . (4) Nav1.8 expression in the L4-6 DRG was evaluated by using of RT-PCR and Q-PCR methods $24 \mathrm{~h}$ after the last siRNA injection.

Results: Rats receiving intra-tibial injections of Walker256 cells displayed gradual development of mechanical allodynia and thermal hyperalgesia, beginning from day 7 following injection of cells. Intrathecal injection of M-siRNA attenuated mechanical allodynia and thermal hyperalgesia of bone cancer pain and was concomitant with a reduction in the Nav1.8 transcripts in the lumbar DRG. Negative control RNA injection did not alter expression of Nav1.8 in lumbar DRG, and had no effect on the baseline threshold of nociception. The antinociception effect of M-siRNA continued to $72 \mathrm{~h}$ after the last RNA injection.

Conclusion: We conclude that silencing of Nav1.8 channel using a siRNA approach is capable of producing allodynia and hyperalgesia relief in the bone cancer model.

\section{P 075}

\section{Upregulation of $\mathrm{P} 2 \mathrm{X} 3$ receptor in dorsal root ganglia of a cancer pain model}

J. Y. G. Jiang, X. Longhe, Z. Hong

PLA General Hospital, Department of Anesthesiology, Beijing, China

Background and objective: $\mathrm{P} 2 \mathrm{X} 3$ purinergic receptors are predominantly expressed in dorsal root ganglion (DRG) neurons and play an important role in pain sensation. Changes of P2X3 receptor in DRG of cancer pain remains unclear. The aim of this investigation was to induce bone cancer pain in rats, and to observe the expression of $\mathrm{P} 2 \mathrm{X} 3$ receptor in cancer pain models. Methods and materials: Female SD rats recieved intra-tibial injection of 5103,5104 or 5105 syngenetic Walker256 mammary gland carcinoma cells. Mechanical allodynia and thermal hyperalgesia were tested at 1 day, 3 days, 5 days, 7 days, 10 days and 14 days after cell injection. The development of the bone tumour and metastasis was monitored by histology. The L5-6 DRG were obtained from cancer pain rats. Expression of P2X3 receptor was investigated by RT-PCR and western blot. Result: Intra-tibial injections of Walker256 cells produced a rapidly expanding tumour within the boundaries of the tibia, causing severe remodelling of the bone. No tumour was observed in the contralateral tibia. But rats receiving 5105 
Walker256 cells showed significant body weight loss. One rat from this group developed lymph nodes and lung matastasis. Rats receiving intra-tibial injections of Walker256 cells displayed gradual development of mechanical allodynia and thermal hyperalgesia, beginning from day 7-14 following injection of cells. These symptoms were not observed in rats receiving normal saline. The mRNA and protein expression of $P 2 X 3$ receptor in DRG were upregulated in cancer-induced nociception. The increases were seen ipsilaterally.

Conclusion: The induction of bone cancer in rats by the syngeneic Walker256 mammary tumour cell line provided a valid pre-clinical model for pain associated with bone metastases. Bone cancer pain resulted in an upregulation in the expression of $\mathrm{P} 2 \mathrm{X} 3$ receptor in DRG, suggesting that $\mathrm{P} 2 \mathrm{X} 3$ receptor may contribute to cancer pain.

\section{P 076}

\section{Effects of wound infiltration with levobupivacaine and levobupivacaine-methylprednisolone on postoperative pain in lumbar discectomy}

G. A. Gurbet, A. Bekar, H. Bilgin, G. Korfali, M. Tercan,

S. Yilmazlar

Uludag University, Medical Faculty, Department of Anesthesiology and Reanimation, UUTF Anesteziyoloji ve Reanimasyon $A D$,

Bursa, Turkey

Background and objective: In this prospective, randomized, controlled trial we aimed the efficacy of preemptive wound infiltration with levobupivacaine and levobupivacaine-methylprednisolone after lumbar discectomy.

Methods and materials: One hundred patients were randomly allocated to five equal groups as follows: Group I had the musculus multifidi near the operated level infiltrated with $30 \mathrm{~mL}$ $0.25 \%$ levobupivacaine and $40 \mathrm{mg}$ methylprednisolone just before wound closure; Group II had the same region infiltrated with $30 \mathrm{~mL}$ 0.25\% levobupivacaine alone before closure; Group III had this region infiltrated with $30 \mathrm{~mL} 0.25 \%$ levobupivacaine and $40 \mathrm{mg}$ methylprednisolone before the incision was made (preemptive); in Group IV, this region infiltrated with $30 \mathrm{~mL} 0.25 \%$ levobupivacaine alone before incision (preemptive); and Group C (control) had this region infiltrated with $30 \mathrm{~mL} 0.9 \%$ $\mathrm{NaCl}$ just before wound closure. Patient charactersitics data, vital signs, postoperative pain scores, and morphine usage were recorded.

Results: All four groups treated with levobupivacaine or levobupivacaine-methylprednisolone (by pre-emptive or preclosure wound infiltration) showed significantly better results than the control group for most parameters. The treated groups had lower parenteral opioid requirements after surgery, lower incidences of nausea, and shorter hospital stays. Further, the data indicate that, compared with infiltration of these drugs at wound closure, preemptive injection of levobupivacaine or levobupivacaine-methylprednisolone into muscle near the operative site provides more effective analgesia after lumbar discectomy.

Conclusion: In addition, our data suggest that preemptive infiltration of the wound site with levobupivacaine alone provides similar pain control to preemptive infiltration of the wound site with levobupivacaine and methylprednisolone combined.

\section{P 077}

\section{Perioperative pain in neurosurgery - an audit}

R. Sindhu, Z. Ali, L. Colville, J. Golding

Walton Neuro Centre, Department of Anaesthesia, Horsley Itu, Walton Centre, Liverpool, UK
Background and objective: Effective pain management is a fundamental quality of care issue for surgical patients and influences perioperative morbidity 1,2 . The aim was to assess: (1) differing perioperative analgesia practice; (2) quality of analgesia in first $24 \mathrm{~h}$ in a busy neurosurgical unit; (3) any areas of concerns against The Royal College of Anaesthetists recommendations.

Methods and materials: Data collected prospectively for 3 months from elective surgical patients in the first $24 \mathrm{~h}$ postoperatively. Data included patient charactersitics data, type of procedures, perioperative and postoperative analgesia received, pain scores on visual analogue scale $(0-10)$ on arrival, at $30 \mathrm{~min}$ and at discharge from recovery, ward observations and patient satisfaction.

Results: Complete data was obtained from 180 patients (36.7\% ( $n=491$ ) of all elective patients) and results analysed in four sub groups namely craniotomy, spinal surgery, endovascular procedure and others (trans-sphenoidal and shunt surgery, etc.). Multimodal analgesia was used intraoperatively for the majority of patients. In all, $30 \%$ of craniotomy, $40.6 \%$ of spinal surgery and $20 \%$ of endovascular patients were in moderate to severe pain on arrival in recovery. Hesitancy in use of opioids and patients with chronic pain syndromes may explain these high pain scores. In all, $4.76 \%$ of craniotomy, $2.1 \%$ of spinal surgery and $0 \%$ of endovascular patients had pain scores $>3$ on discharge from recovery.

We were unable to assess the effectiveness of postoperative analgesia as no pain score system was in practice. Patient satisfaction with analgesia averaged $84.5 \%$.

Conclusion: (1) Increased use of intraoperative analgesics needs to be encouraged to decrease the percentage of patients arriving in recovery with moderate to severe pain. (2) There may be a place for increased use of NSAIDs, patient controlled analgesia and local techniques. (3) Discharge criteria from the recovery needs to include pain scores of $\leqslant 3$. (4) Pain scoring needs to be initiated to assess the effectiveness of postoperative analgesia regimens.

\section{P 078 \\ Long-duration pulsed radiofrequency for the treatment of upper extremity complex regional pain syndrome}

G. Lipov, R. Joshi

Alexian Brothers Hospital Network Pain Program, Department of Anesthsiology, USA

Background and objective: Complex regional pain syndrome type I (CRPS I) that has persisted for more than 6 months is generally unresponsive to conventional treatments, including sympathetic and regional anaesthetic blocks, and only partially responsive to implants. We found, however, that pulsed radiofrequency (PRF) was highly effective in this patient population, especially in those with upper extremity CRPS. The aim was to test a protocol of PRF of the stellate ganglion in patients with upper extremity CRPS I who had undergone numerous treatments without lasting relief.

Methods and materials: To date, we have treated seven patients with the following protocol: after placing an insulated needle with active tip near the stellate ganglion at the C6 level, we delivered pulses of $500-\mathrm{kHz}$ current at 2 bursts per second for 10 min.

Results: All patients experienced immediate pain reduction $(\geqslant 80 \%)$ and elimination of hyperaesthesia. The duration of these effects was 48-150 days. Two patients had successful second PRF treatments. Incidentally noted by three patients (heavy smokers) were diminished cravings for cigarettes and a decrease 
in their smoking. Two menopausal patients reported a reduction in hot flashes.

Discussion: Although CRPS was once thought to be entirely sympathetically mediated, there is a growing body of evidence implicating central mechanisms in its pathophysiology. The stellate ganglion's sphere of innervation has been shown to extend beyond the sympathetic nervous system and to include key central structures including the insular cortex. While PRF's mechanism is only partially understood, its electrical fields may interrupt key connections between the peripheral and central nervous systems. The reductions in smoking and hot flashes were not surprising, as the insular cortex has been shown to play a key role in smoking addiction and to be active during hot flashes.

Conclusion: PRF, a minimally invasive technique, appears to offer substantial benefit to patients with chronic CRPS.

\section{ELECTROPHYSIOLOGY/EPILEPSY/MONITORING}

\section{P 079}

\section{The use of near-infra-red spectroscopy (NIRS) in surgical clipping of giant cerebral aneurysm}

Dr. Khalifah, R. Craen ${ }^{\dagger}$, D. Bainbridge ${ }^{\dagger}$, S. Lownie ${ }^{\dagger}$, M. Quantz ${ }^{\dagger}$, J. MacDonald

${ }^{*}$ King Faisal Specialist Hospital, Department of Anesthesia, Riyadh, Saudi Arabia; ' London Health Sciences Centre, London, Ontario, Canada

Background and objective: Surgical management of giant intracranial aneurysms is challenging to the surgeons and anaesthesiologists. We report the use of near infra-red spectroscopy (NIRS) and electroencephalograph (EEG) to detect and monitor signs of cerebral ischaemia during surgical clipping of giant aneurysm.

Methods and materials: Utilizing right femoral to right carotid artery extracorporeal perfusion combined with NIRS and EEG.

Results: NIRS was a useful additional neuromonitoring modality that allows early detection of cerebral ischaemia and thus early intervention.

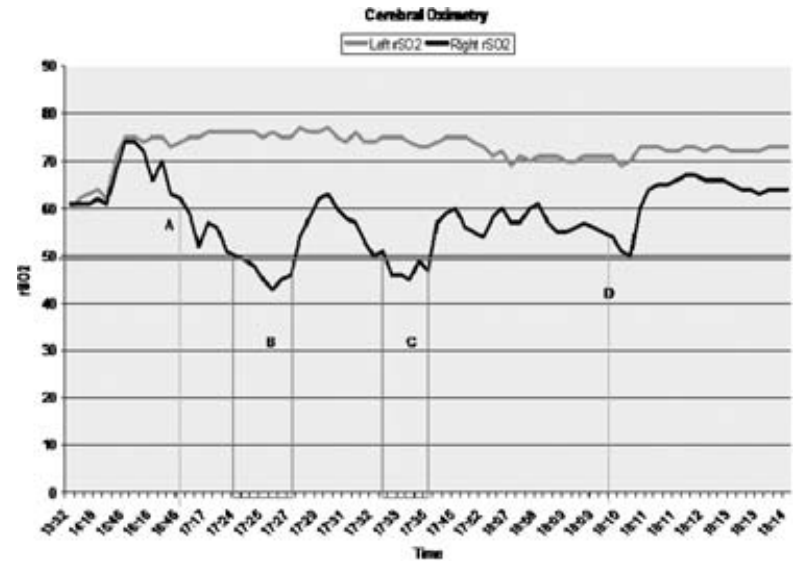

Figure.

Conclusion: This technique seems to be promising may carry less intraoperative, postoperative complications, and may help guide therapeutic decisions.

\section{P 080}

\section{Quality of life in epileptic patients}

S. Shaafi, M. N. Nikanfar

Tabriz University of Medical Science, Department of Neurology, Neurology Ward - Razi Hospital, Tabriz, Iran
Background and objective: Epilepsy is a chronic disease, which has a major influence on the quality of life in the epileptic patient. The aim of this research was to study the quality of life of the epileptic patients in our society, and compare with that of other centres all around the world.

Methods and materials: In all, 189 patients were enrolled according to the criteria of the International League Against Epilepsy (ILAE) for diagnosis of epilepsy. The patients selected accordingly were interviewed using a standard questionnaire, Quality Of Life In Epilepsy (QOLIE-31-P).

Results: Among 189 patients, 106 were males (56.1\%) and 83 were females $(43.9 \%)$. Total quality of life according to the score of QOLIE-31-P was minimally 16.20 and maximally 87.5 mean of $(50,14.59 \mathrm{SD})$. The mean score for males was 50 (16.11 SD; 16.2-85.35) and for females 49.28 (14.47 SD; 24.14-87.5), with no significant difference between sexes $P$ value $=0.549$. The mean score in the monotherapy group $(52.95,16.22 \mathrm{SD})$ with a significant difference with poly therapy group a mean score of $(46.98,12.07 \mathrm{SD}),(P$ value $=0.005)$. There was no significant difference statistically with regard to age groups and educational status, but duration of the epilepsy less than $1 \mathrm{yr}$ had statistically significant better quality of life.

Conclusion: The findings on the role of the sex of the patients was the same as the studies in the India and Georgian University, but were different to that of a European study of 15 countries. This may stem form cultural differences and beliefs. The effect of number of drugs used in treatment was similar to other studies. The care of the epileptic patients must take into account the many factors influencing the quality of life in these patients.

\section{P 081}

\section{Alternative therapy improving quality of life in epilepsy} A. Singh, K. Dalal, M. Bhart

All India Institute of Medical Sciences, Department of Biophysics, New Delhi, India

Background and objective: Treating epilepsy with alternative therapies. This study aims at managing patients suffering from intractable epilepsy applying indirect vagus nerve stimulation (indirect VNS) and reflexology.

Methods and materials: This is a $3 y$ r randomized two-arm pilot study. The group in arm one is treated with pharmacological drugs, reflexology therapy and indirect VNS. The second arm comprising the control group continues on anti-epileptic drug therapy. Patients with age group from 3 to $60 \mathrm{yr}$, who had intractable epilepsy for at least $3 \mathrm{yr}$ and met the inclusion criteria, have been enrolled. They were allotted to the study groups using standard randomization technique. Baseline and yearly follow-up of bio-chemical and physiological parameters are being used to evaluate health status of patients. QOLIE-31 is used to assess the behavioural patterns and quality of life every 6 months. Engel score was used to evaluate the status of seizure. 
Results: A total of 23 patients have been admitted to the study till March 2007 (sample size of study group = 13 and, sample size of control group $=10$ ). The response of the patients to the therapy depended on good compliance. In all, $80 \%$ of the patients showed a positive response with regards to reduction in seizure frequency, duration of ictal phase and improvement in behavioural patterns of patients, at the end of 4-month monitoring period.

Conclusions: Patients will be monitored for a period of $3 \mathrm{yr}$. Unique response of the sample group to indirect VNS reveals the effectiveness of this technique in managing refractory epileptic patients with least side-effect and least cost, applicable at any place and any time in a non-pharmacological way but with similar results as obtained in an expensive procedure of direct vagus nerve stimulation implanted electrical stimulators. Hence, this complimentary medicine maintains uninterrupted personal and family lives.

\section{P 082}

The value of measuring current perception threshold in diabetic sensory polyneuropathy with normal routine NCS

O. S. H. Oh*, J. J. Sung ${ }^{\dagger}$, Y. H. Hong ${ }^{\dagger}$, S. H. Park*,

K. W. Lee ${ }^{\dagger}$, K. S. Park ${ }^{\star}$

*Seoul National University Bundang Hospital, Department of Neurology, Gyeonggi-Do; 'Seoul National University, Seoul, South Korea

Background and objective: Sensory polyneuropathy is one of the most common neurological complication of diabetes mellitus. Routine nerve conduction study (NCS) is sometimes normal in diabetic patients with clinical manifestations of sensory polyneuropathy, especially in the early stage. We report on the value of measuring current perception threshold (CPT) in diabetic sensory polyneuropathy with normal routine NCS.

Methods and materials: Thirty diabetic patients with sensory polyneuropathy with normal routine NCS (13 males, 17 females, aged $55-80 \mathrm{yr}$, mean $66.33 \pm 7.12)$ and 19 non-diabetic control subjects ( 7 males, 12 females, aged 51-68 yr, mean $58.68 \pm 5.65$ ) participated in this study. We measured CPT by using Neurometer (Neurotron Inc., USA) on the index finger and great toe of each patient at the frequency of 2000,250 and $5 \mathrm{~Hz}$.

Results: Compared with the control group, the polyneuropathy group showed significantly high CPT at the frequency of $2000 \mathrm{~Hz}$ $(P=0.041)$ in the hands, and $250 \mathrm{~Hz}(P=0.006)$ and $5 \mathrm{~Hz}$ $(P=0.004)$ in the feet. Closer correlation was observed between 250 and $5 \mathrm{~Hz}$, and 2000 and $250 \mathrm{~Hz}$ than between 2000 and $5 \mathrm{~Hz}$, both in the hands and in the feet.

Conclusion: Measuring CPT is useful for detecting sensory abnormalities in diabetic sensory polyneuropathy. It is a simple, sensitive, and objective means even in the patients with normal routine NCS.

\section{P 083}

\section{Effect of partial neuromuscular block on intraoperative facial nerve monitoring during excision of vestibular schwannoma}

\section{T. V. Chan, N. Gin}

The Chinese University of Hong Kong, Department of Anaesthesia and Intensive Care, Nil Hong Kong Sar, Hong Kong

Background and objective: Intraoperative facial nerve monitoring provides early detection of nerve injury during surgery. Traditionally, neuromusclar blocking agent is avoided to prevent interference with monitoring. However, more recent study suggested that partial neuromuscular blockade could be used without compromise of monitoring while avoiding large doses of anaesthetics. In this study, we compared the perioperative anaesthetic and facial nerve outcomes in patients receiving partial neuromuscular block with those treated with no neuromuscular blocking agent during excision of vestibular schwannoma.

Methods and materials: The study was approved by the clinical Ethics Committee. Written informed consent was obtained from 120 patients. Anaesthesia was maintained with target-controlled infusions of propofol and remifentanil. Following a bolus dose of rocuronium and tracheal intubation, patients were randomly allocated to receive either no further relaxant or an infusion of rocuronium to maintain $50 \%$ first twitch suppression. Facial nerve monitoring was accomplished by placement of subdermal electrodes in the orbicularis oris and occuli muscles. Intraoperative feasibility of monitoring, anatomical preservation of the facial nerve, anaesthetic doses administered and haemodynamic parameters were recorded. Postoperative facial nerve function was rated using the six-point House and Brackmann $(\mathrm{H}-\mathrm{B})$ scale ( $1=$ normal function, $6=$ total paralysis) at discharge and 6 months after surgery.

Results: The mean \pm standard deviation target plasma concentration of propofol in the rocuronium group, $3.1 \pm 0.4 \mu \mathrm{g} \mathrm{mL}^{-1}$, was similar to that of the non-relaxant group, $3.2 \pm 0.3 \mu \mathrm{g} \mathrm{mL}^{-1}$. The median (range) $\mathrm{H}-\mathrm{B}$ scores in the rocuronium group, 3 (1-6) at discharge and $2(1-6)$ at 6 months, were worse compared with the non-relaxant group, $2(1-5)$ and $1(1-5), P=0.03$, respectively.

Conclusions: Partial neuromuscular block impaired the integrity of facial nerve monitoring. Repeated injury may be missed while monitoring with partial neuromuscular block. This may contribute to worsen facial nerve function after excision of vestibular schwannoma.

\section{P 084}

\section{Prevalence and electrophysiologic evaluation of carpal tunnel syndrome in pregnant women}

Sh. Shaafi

Tabriz University of Medical Science, Department of Neurology, Neurology Ward - Razi Hospital, Tabriz, Iran

Background and objective: Carpal tunnel syndrome is entrapment of median nerve in carpal tunnel of the wrist. This syndrome is associated with many conditions such as pregnancy which is one of the most frequent conditions. This syndrome can be diagnosed by manifestations and electroneurodiagnostic studies. The aim of this study is to determine frequency and severity of CTS by electroneurodiagnostic studies in pregnant women. Also it attempts to clarify the role of some factors such as age, gravity, trimester of pregnancy and body mass index in patients with CTS. Finally, it compares symptomatic and asymptomatic females with electroneurodiagnostic studies.

Methods and materials: A convenience sample of 90 pregnant women ( 30 women from each trimester). All of the cases have been examined clinically and then by electroneurodiagnostic tests.

Results: There were suspected symptoms of CTS in 45 patients. The most frequent symptoms and signs were hands paraesthesia (34\%) and positive tinel and phalen sign (each $22 \%$ ). CTS was definite diagnosis in 15 females (16.6\%) by electroneurodiagnostic studies. Mild bilateral involvement was seen in $73.3 \%$ of patients. There was a meaningful relationship between CTS and trimester that $26.6 \%$ of patients were at third trimester. Also there was a meaningful relation to age. The CTS was more frequent in older females. BMI and gravity relationships with CTS were not meaningful. 
Conclusion: CTS is frequent in pregnancy and this frequency increases in older females and in third trimester. Fortunately, CTS during pregnancy is often mild and relieves with conservative treatments. More attention must be paid to electroneurodiagnostic studies in this patients for definite diagnosis and assure the patients that it is a benign and curable disturbance.

\section{P 085}

The effect of prone position on respiratory mechanics during spinal surgery

E. Manaa

King Khalid University Hospital, Department of Anesthesia (41),

Riyadh, Saudi Arabia

Background and objective: To study the effect of prone position on respiratory mechanics during spine surgery.

Methods and materials: Prospective study. Elective spine surgery at a university hospital. In all, 12 ASA physical I \& II patients with no coexisting cardiorespiratory disease, undergoing cervical or lumbar laminectomy under general anaesthesia in prone position. Ten minutes after induction of general anaesthesia and endotracheal intubation, while patients were in supine position, the following measurements were taken using anaesthesia delivery unit (Datex Ohmeda type A_Elec, Promma, Sweden): peak airway pressure (Ppeak), peak plataeu pressure (Pplat), peak mean pressure (Pmean) and dynamic lung compliance (DLC). The same measurements were recorded $10 \mathrm{~min}$ after placing patients into prone position. At the end of surgery and $5 \mathrm{~min}$ after turning the patients supine and before tracheal extubation, the same measurements were again recorded. The results expressed as mean $\pm S D$. One-way ANOVA was used for analysis of differences in the data before, during prone position and after turning patients supine at the end of the procedure. For all comparisons $P<0.05$ was considered significant.

Results: During prone position there was significant reduction in DLC and significant increase in airway pressures.

Conclusion: We concluded that turning the patients from supine to prone position during anaesthesia for spine surgery caused significant decrease of DLC and significant increase of airway pressure.

\section{FUNCTIONAL NEUROSURGERY}

\section{P 086}

\section{Vagus nerve stimulation in treatment of seizure}

S. A. Singh, S. Singh

All India Institute of Medical Sciences, Department of Biophysics, New Delhi, India

Background and objective: Vagus nerve stimulation (VNS) is novel adjunctive therapy that has recently become commercially available for intractable epilepsy. Age group $15-65 \mathrm{yr}$, who had intractable epilepsy for at least $3 \mathrm{yr}$, met the inclusion criteria. This study aims at managing patients suffering from intractable epilepsy by applying vagus nerve stimulation.

Methods and materials: The VNS system is comprised of battery generator that delivers electrical stimuli via programmed software. The electrode is implanted in neck and connected to left vagus nerve. This is a $3 \mathrm{yr}$ randomized two-arm pilot study. The group in arm one is treated with pharmacological drugs, and VNS. The second arm comprising the control group continue on anti-epileptic drug therapy.

Results: A total of 35 patients have been admitted to the study till March 2007 (sample size of study group $=17$ and, sample size of control group $=18$ ). The response of the patients to the therapy depended on good compliance. Of the patients, $80 \%$ showed a positive response with regards to reduction in seizure frequency, duration of ictal phase and improvement in behavioural patterns of patients, at the end of 4 months monitoring period.

Conclusions: This is an ongoing study and the patients will be monitored for a period of 3 yr. Unique response with VNS reveals the effectiveness of this technique in managing refractory epileptic patients with least side-effect and cost, but with similar results as obtained in an expensive and invasive surgical resection.

\section{P 087}

\section{The effect of surgical nerve repair on survival of primary sensory neurons}

Dr. Atlasi ${ }^{\star}$, Dr. Roozbehi ${ }^{\dagger}$, Dr. Farjah"

*Kashan University of Medical Sciences, Department of Anatomy, Kashan University of Medical Sciences, Kashan; ${ }^{\dagger}$ Yasooj Uiversity of Medical Scinences, Yasooj; "Oromieh University of Medical Sciences, Oromieh, Iran

Background and objective: Peripheral nerve transection causes neuronal cell death. For demonestration of effects of peripheral surgical repair methods on primary sensory survival, this study carried out and compared two surgical repair techniques of autograft and repair with using of nerve guidance channel.

Methods and materials: This is experimental study on dorsal root ganglion neurons of adult rats by Nissle staining. Models of axotomy, autograft, and using of nerve guidance channel of polyvinilydin flourid (PVDF) were used and neuronal number dorsal root ganglion of L5 was evaluated. In the first group sciatic nerve in mid-thigh transect and $1 \mathrm{~cm}$ of sciatic nerve removed, in second group $1 \mathrm{~cm}$ of the nerve removed and sutured after $180^{\circ}$ rotation. In the third group $1 \mathrm{~cm}$ of sciatic nerve in mid-thigh removed and replaced by PVDF tube. After 3 months, rats were perfused and fifth left dorsal root ganglion extarcted. The DRGs sectioned serially at $20 \mu \mathrm{m}$ by cryostate and mounted on gelatine slides and stained by $0.1 \%$ Nissle dye. At the end, normal neurons counted by graticule lens and analysied by $t$-test. Also morphology of neurons studied by light microscopy.

Results: Average of normal neurons in axotomy group was $27.42 \pm 7$, in autograft group was $42.1 \pm 3.33$ and in PVDF group was $34.82 \pm 3$. Although mean number of normal neurons in PVDF group was lesser than autograft group, but was not significant. Conclusion: Sciatic nerve repair by autograft and using of nerve guidance channel of PVDF methods cause to decrease of sensory neuronal loss, but cannot stop this process. 


\section{P 088}

Intrinsic properties of axonal regeneration in chronic spinal cord injury model

H. N. Hong Hong, Y. J. Won Won, J. Y. Yoo Yoo, N. R. Bae Bae

Ulsan University School of Medicine, Department of Anatomy and Cell Biology, Seoul, South Korea

Background and objective: Numerous experiments for spinal cord regeneration have been conducted using different injury model and techniques. Although many studies have shown some effective strategies in acute model within weeks after spinal cord injury (SCl), little is known about the applicability in chronic injury model. In this study, we investigated intrinsic properties of spontaneously regenerated spinal cord from the chronic model whose injury occurred 13-15 months ago.

Methods and materials: The spinal transecting lesion was made at T10 level with SD rats then housed for 13-15 months. The spontaneous recovery of gross overground locomotion was evaluated once per week thereafter using the Basso-BeattieBresnahan (BBB) rating scale. Animals were excluded from further analysis if the locomotor function was normalized 1-2 weeks after injury. After 13-15 months, the animal group was divided two groups, spontaneous recovered (SR) group (18-21 level of BBB) and chronic-injured (Cl) group (1-3 level of BBB). To explore the contribution of reactive astrocyte and glial environment on the spontaneous axonal regeneration, the level of GFAP, MBP (myelin basic protein), phosphocan, O4, RIP (for oligodendrocytes), GAP-43, PCNA (proliferating cell nuclear antigen), NeuN, SMI-32 expression was examined on the rostral, epicentre, and caudal segments by immunohistochemistry and western blot analysis.

Results: While GFAP level was reduced in $\mathrm{Cl}$ group especially in the caudal part, increased GFAP was observed in SR group accompanying significant decrease in the expression of MBP.

Conclusion: Our results suggest that reactive astrocytes provide a permissive environment for spontaneous axonal regeneration in the chronic spinal cord injury model.

\section{NEUROCRITICAL CARE NURSING}

\section{P 089}

\section{Examination of the effect of lidocaine on intracranial pressure during endotracheal suctioning in severe head-injured patients in Shiraz-Iran}

S. Gholamzadeh, S. Rahnama, M. Javadi, A. Haqnegahdar, B. Zeighami

Shiraz Medical University, Department of Fatemeh Nursing and Midwifery College, Shiraz, Iran

Background and objective: Endotracheal suctioning is a routine nursing procedure used to decrease pulmonary complications; however, in severe head-injured patients it can result in sudden increase in intracranial pressure and may put the patients at risk for further cerebral damages. The purpose of this prospective, repeated measure within-group study was to examine the effect of lidocaine administration on ICP during ETS in severe headinjured patients.

Methods and materials: Thirteen patients with acute severe head injury (Glasgow Coma Score 4-8) were studied. Each subject received suctioning without and with doses of 0.75 and $1.5 \mathrm{mg} \mathrm{kg}^{-1}$ intratracheal lidocaine administration. ETS protocol consisting of administration of 16 breathe at $135 \%$ of patients' tidal volume, $100 \% \mathrm{FiO}_{2}$ before and after suctioning with standardized catheter (16 French) and duration about $10 \mathrm{~s}$. Repeated measure model for ANOVA were used to examine the changes in mean ICP at $5 \mathrm{~min}$ before, during, and $2 \mathrm{~min}$ after ETS.

Results: Changes in ICP significantly smaller in method of suctioning with $1.5 \mathrm{mg} \mathrm{kg}^{-1}$ lidocaine administration than other two methods of suctioning with $0.75 \mathrm{mg} \mathrm{kg}^{-1}$ lidocaine or without its administration. The three methods showed no significant difference in the return of ICP to baseline level.

Conclusions: Change in ICP induced by ETS in severe head-injured patients is moderated by $1.5 \mathrm{mg} \mathrm{kg}^{-1}$ of intratracheal lidocaine administration, but $0.75 \mathrm{mg} \mathrm{kg}^{-1}$ intratracheal lidocaine cannot attenuate the increase in ICP that occur with ETS.

\section{P 090}

To evaluate the correlation of nosocomial pneumonia and the administration of antacids/H2-antagonists in Neurosurgical ICU patients in Shiraz-Iran

M. S. Gholamzadeh, M. Moatary

Shiraz Medical University, Department of Fatemeh Nursing and Midwifery College, Shiraz, Iran

Background and objective: There is some evidence that acidsuppressive therapy facilitates nosocomial infections. H2-receptor antagonists and proton pump inhibitors (PPIs) increase susceptibility to infections by increasing gastric $\mathrm{pH}$. Microbiological studies have shown that a gastric $\mathrm{pH}>4$ is crucial for overgrowth of gastric gram-negative.

Methods and materials: This descriptive study attempted to identify the correlation of nosocomial pneumonia and the administration of antacids/ $\mathrm{H} 2$-antagonists in mechanically ventilated patients admitted to the neurosurgery ICU in Shiraz teaching hospitals - Iran. Thirty critically ill patients who did not have pneumonia on admission were evaluated for 5 days. Pneumonia was diagnosed according to the presence of those criteria: fever $38^{\circ} \mathrm{C}$, increase in blood leucocyte count, new and progressive infiltrate on chest radiograph, and positive sputum culture result. Periodically gastric aspirations were collected measuring gastric $\mathrm{pH}$ during the study periods.

Results: The mean age of the patients was $23 \pm 12.5$ (range, $5-40 \mathrm{yr}$ ) with a male/female ratio of $22 / 8$. Majority of patients (24, $80 \%$ ) were head trauma. Four patients had brain tumour and two patients had subarachnoid haemorrhage. The pneumonia incidence was $40 \%$ during the study period (5 days). Pneumonia presented in all of patients who received antacid. There was no statistically significant difference in pneumonia rates and $\mathrm{H} 2$ antagonists prophylaxis. Mean gastric $\mathrm{pH}$ during the study was $4.56 \pm 1.52$. There was no correlation between gastric $\mathrm{PH}$ and pneumonia.

Conclusion: Based on the findings, the use of antacids is associated with a higher incidence of nosocomial pneumonia in comparison to histamine-2-receptor antagonists. 


\section{P 091}

\section{Patient satisfaction in awake craniotomy}

Dr. Khalifah*, I. Herrick ${ }^{\dagger}$, A. Parrent ${ }^{\dagger}$, J. Megyesi ${ }^{\dagger}$, R. Craen ${ }^{\dagger}$

${ }^{*}$ King Faisal Specialist Hospital, Department of Anesthesia, Riyadh, Saudi Arabia; ' London Health Sciences Centre, London, Ontario, Canada

Background and objective: Awake craniotomy using local anaesthesia and monitored conscious sedation is widely used for the excision of intracranial tumours or vascular abnormalities or for the management of refractory seizures. Propofol combined with remifentanil represents a popular technique for the provision of conscious sedation during these procedures. This study evaluated patient satisfaction following awake craniotomy performed under propofol-remifentanil sedation. The study also assessed the incidence of intraoperative and postoperative complications associated with this technique.

Methods and materials: This prospective study evaluated the satisfaction of 25 adult patients undergoing awake craniotomy under propofol-remifentanil sedation. Evaluation involved interviewing patients at $1 \mathrm{~h}, 24 \mathrm{~h}$ and 6 weeks postoperatively. Postoperative recall of pain, anxiety and discomfort were assessed at $1 \mathrm{~h}, 24 \mathrm{~h}$ and 6 weeks postoperatively. Surgeon and anaesthesiologist satisfaction was also evaluated at the end of each procedure.

Results: At $1 \mathrm{~h}$ postoperative assessment, 24 patients (96\%) were satisfied with the anaesthetic technique. Patient satisfaction scores were similar at $1 \mathrm{~h}, 24 \mathrm{~h}$ and 6 weeks postoperatively. Twenty-one of the 24 patients (84\%) stated that they would choose the same anaesthetic technique if they were to undergo the same procedure again. Surgeons and anaesthesiologists were satisfied in 23 cases (92\%).

Conclusion: This study confirms that monitored conscious sedation with propofol-remifentanil is a useful alternative technique for awake craniotomy with a high patient, surgeon and anaesthetist satisfaction.

\section{P 092}

Is there any relation between echocardiographic patterns of atrial septal defect (ASD) and migraine?

A. Providência, C. S. Silva, M. P. Costa, N. P. Quintal,

I. M Luzeiro, A. M. Leitao-Marques

Centro Hospitalar de Coimbra, Department of Serviço de Cardiologia, Coimbra, Portugal

Background and objective: We have determined the prevalence and type of headache in a series of patients with ASD and evaluated if there is a higher prevalence or severity of neurological complaints in certain echocardiographic patterns of ASD.

Methods and materials: Population was composed of 88 patients with ASD previously studied by transthoracic and transesophageal echocardiography, with average age of $44.7 \pm 18.1 \mathrm{yr}$, with $56(63.6 \%)$ females, assessed about the presence of headache, with respective evaluation by a numeric scale and the Migraine Disability Assessment Questionaire (MIDAS). The presence or absence of headache, and MIDAS score, were compared to the echocardiographic morphology of the ASD. Patients with headache were re-evaluated and symptoms characterized by a single neurologist.

Results: In all, 36 patients $(40.9 \%)$ had regular complains of headache, with $28 / 36(77.8 \%)$ being females. Of these patients, $11 / 36$ had previously been studied in other situation by neurology or previously underwent brain CT scan. In all, 23 (26.1\%) were diagnosed migraine (4 with aura), 12 with tension headache and 1 with medication abuse headache. Prevalence of migraine in this population is 2.2 times superior to the reported in our country $(12.1 \%)$, and the prevalence of tension headache does not differ from general population (from $25 \%$ to $60 \%$ ). MIDAS score in 14 patients was stage I, stage II in 11, stage III in 5 and stage IV in 6 patients. Six different morphologic patterns were identified by echocardiographic evaluation, with $n>5$. There were no significant differences in the prevalence of migraine in different morphologic echocardiographic patterns. Diameter of the defect was not different when comparing patients with and without migraine $(14.7 \mathrm{~mm}$ vs. $15.4 \mathrm{~mm}, P=0.37)$. For each echocardiographic pattern, the MIDAS score was similar.

Conclusion: We conclude that migraine is more prevalent in patients with ASD. More studies will be needed in order to clarify the mechanism of this association.

\section{P 093}

Can the short aortic rim be a risk factor for developing migraine after percutaneous closure of secundumtype atrial septal defect?

\author{
A. Providência, C. S. Silva, M. P. Costa, N. P. Quintal, \\ I. M. Luzeiro, A. M. Leitao-Marques \\ Centro Hospitalar de Coimbra, Department of Serviço de \\ Cardiologia, Coimbra, Portugal
}

Background and objective: Recently published studies suggest a relationship between migraine and interatrial shunts. Evidence seems to point towards most patients improving their neurological symptoms after shunt closure. However, for unknown reasons, some of the previously asymptomatic patients develop migraine after shunt closure.

Methods and materials: We have assessed a potential relationship between ecocardiographic morphological pattern of secundum type atrial septal defect (ASD) and the development of migraine after percutaneous closure. In all, 25 consecutive patients in our centre (14 males and 11 females, aged $37.8 \pm 17.7 \mathrm{yr}$ ) with no previous complaints of migraine, underwent percutaneous closure of secundum type atrial septal defect with Amplatzer devices, with previous echocardiographic evaluation. Patients were screened for the development of neurological symptoms in the first year. Those presenting with positive symptoms, were evaluated by a single neurologist.

Results: Three patients (two females and one male) developed headache after percutaneous closure, and fulfilled international criteria for migraine. Two of them were free of complaints at the end of the first year. According to criteria present in the literature, six morphological patterns were found. The three patients developing migraine all had the same pattern - short aortic rim. Migraine development was more prevalent in patients with short aortic rim when compared to other morphologies $(P=0.036)$. There were no significant differences in device diameter in patients developing migraine when compared to the rest of the patients.

Conclusion: Our data raise the possibility that, when using Amplatzer devices, some morphological patterns of ASD may be related to the development of migraine after percutaneous closure.

\section{P 094}

\section{Rapidly diagnosing standard electrocardiogram for emergency treatment}

H. Y. Xu

Xin Ye County People's Hospital, Henan Province, China 
Background and objective: Initiated a new method for rapidly diagnosing standard electrocardiogram for emergency treatment.

Methods and materials: Research and summarize the method for rapidly diagnosing standard electrocardiogram the author done in department of emergency medicine.

Results: The methods of the rapidly diagnosing standard electrocardiogram as following. Which the rightness per cent rate of the methods is nearly $100 \%$. (1) The electrocardiogram machine operator for ECG must be a doctor with rich clinical medical experiences and medical knowledge. (2) The doctor operator should give his whole attention to operate the electrocardiogram machine. When operating, the doctor should concentrate his mind on the moving ECG graphs. Which produces immediately the ECG diagnosis ideas. Then, when the ECG graphs come out of the machine or are on the machine. The doctor operator should prove and mend his former diagnosis ideas. So the rapidly diagnosing standard electrocardiogram is done. (3) The doctor should master and be absolutely clear the common disease's ECG graphs. (4) When operating electrocardiogram machine and diagnosing rapidly emergency ECG, the doctor operator should combine patients' clinical syndromes with ECG and pay special attention to the related disease's ECG diagnosis. (5) The doctor operator should scan swiftly the ECG when just finish the ECG operation to further prove the doctor's rapidly diagnosing standard electrocardiogram for emergency treatment. At this moment, the emergency treatments for the special disease can be done. (6) Usually, the measurement, calculation, looking up the table for ECG diagnosis are not needed for the rapidly diagnosing standard electrocardiogram in emergency diagnosis patient and treatment.

\section{P 095}

\section{Neurosurgery in sitting position: analysis of 155 patients}

G. Korfali, H. Bilgin, A. Atlas

Uludag University, Medical Faculty, Department of Anesthesiology and Reanimation, Bursa, Turkey

Background and objective: The sitting position offers many advantages in terms of surgical access for posterior fossa and posterior cervical spine surgery. The main concern is the risk of venous air embolism (VAE).

Our aim was to evaluate the incidence of VAE in our hospital between February 2004-2007.

Methods and materials: In all, 155 patients, ASA status I-III, of all age groups were included in the study. VAE monitoring consisted of precordial doppler and capnography. In all patients, a single or three lumen central venous catheter was inserted by ECG. VAE was suspected if no air was aspirated but one of the following criteria was fulfilled: sudden decrease in end-tidal $\mathrm{CO}_{2}$ $(\geqslant 3 \mathrm{mmHg})$ and/or sudden decrease in mean blood pressure $(<30 \%)$.)

Results: The mean age was $44.28 \pm 16.78 \mathrm{yr}$ ranging from 3 to $80 \mathrm{yr}$ (64 male 91 female). VAE was detected in 47 of 155 operations $(30 \%)$ and occurred more than once during the same procedure in 14 patients there were 74 separate episodes of VAE. In $91 \%$ of patients, air was aspirable through the central venous catheter. The majority of VAEs were small or moderate size. The incidence of associated hypotension was $14 \%$. In two patients there was a shower of air passing through brachial catheter no obvious entry points found of VAEs. Eleven (23\%) patients' position was changed from sitting to prone to continue the surgery. All VAE episodes responded promptly to treatment and there was no peroperative morbidity or mortality directly attributed to it.
Conclusions: With a proper understanding of the pathophysiology of VAE and the use of sensitive monitoring anaesthesia for sitting position neurosurgery can be provided safely.

\section{References}

1. Harrison EA et al. Br J Anaesth 2002; 88: 12.

2. Domaingue CM. Anaesth IntensCare 2005; 33: 332.

\section{P 096}

\section{Levobupivacaine scalp nerve block: haemodynamics and anaesthesia requirements in supratentorial craniotomy}
G. F. Pardey

H. Naous

Pierre Wertheimer's Neurosurgical and Neurological Hospital, Department of Anesthesia and Critical Care, Bron, France

Background and objective: Scalp nerve block (SNB) is a valuable technique during cranial surgery with anaesthetic and analgesic purposes.

Methods and materials: We compared retrospectively (MarchJuly 2007) the anaesthetic and analgesic requirements during craniotomy in 30 patients how received or not a SNB prior to skull pin placement and skin incision. With standard monitoring, anaesthesia was induced with IV sufentanil, sodium thiopental and cisatracurium, and was maintained with continuous IV infusions of sufentanil, propofol and $50 \% \quad \mathrm{O}_{2} / 50 \% \quad \mathrm{~N}_{2} \mathrm{O}$. SNB was performed $5 \mathrm{~min}$ prior to head pinning by the anaesthesiologist, using a modified Pinosky technique, with $20 \mathrm{~mL}$ of Levobupivacaine $0.5 \%$ and epinephrine 1:200000. After scalp asepsia, supraorbital, suprathroclear, auriculotemporal, greater auricular, greater, lesser, and third occipital nerves were blocked at each side of the head. Invasive mean arterial blood pressure (MAP) and heart rate (HR) were recorded: before and after SNB performance, before and after head pinning and before and after skin incision. If HR or MAP increased by more than $20 \%$ over baseline values at head pinning or skin incision, $5-10 \mu \mathrm{g}$ of sufentanil bolus were administrated; if HR or MAP remained elevated, $80-100 \mathrm{mg}$ of IV propofol were also administrated. Haemodynamics, anaesthesia maintenance doses and interventions were compared between groups.

Results: Significant increases in PAM $(P=0.005)$ occurred during head pinning and skin incision relative to baseline values

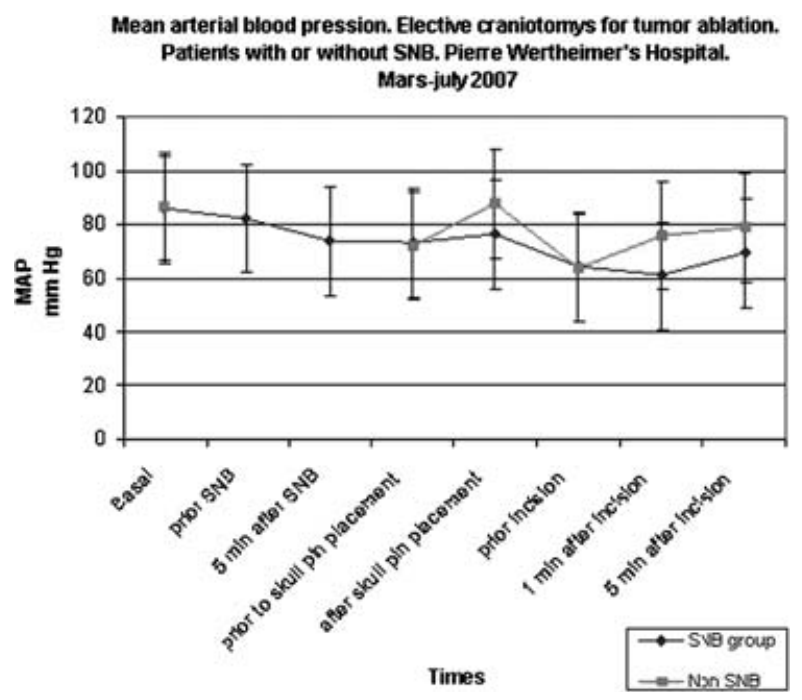

Figure 1. Mean arterial blood pressure. 


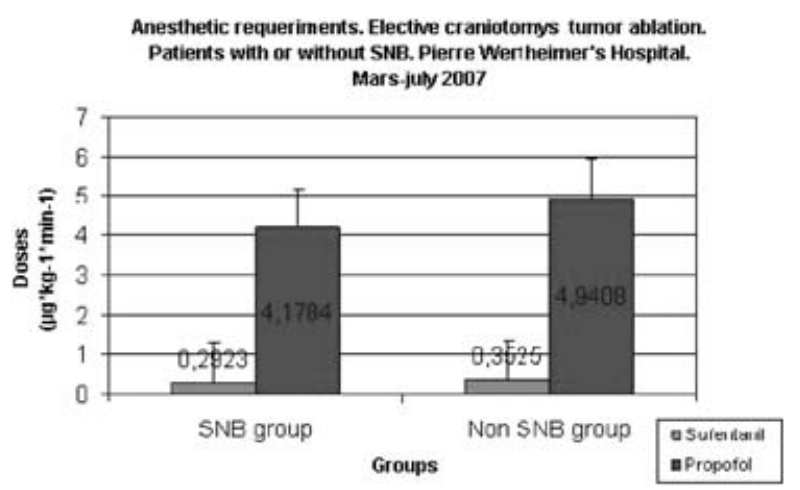

Figure 2. Anaesthestic requirements.

in the group without SNB. Three patients of SNB group vs. fourteen without SNB required additional manoeuvres to control the sympathetic response to head pinning. One patient of SNB group vs. nine without SNB required drugs bolus to control the sympathetic response to skin incision.

Conclusions: Levobupivacaine SNB successfully blunts the haemodynamic response to head pinning and skin incision, provided a great haemodynamical stability during surgery, and also produced anaesthesia and analgesia reducing opioids (17\%) and propofol $(15 \%)$ doses during surgery.

\section{P 097}

Primary-type headaches associated with ribavirin plus interferon alpha-2b therapy in patients with chronic hepatitis $\mathbf{C}$

Y. Jahangiri Noudeh*, S. M. Alavian ${ }^{\dagger}$, A. Sahebjamee*

*Iran University of Medical Sciences, Department of

Medical Students Research Committee; 'Department of

Gastroenterology, Baqiyyatollah University of Medical Sciences,

Tehran, Iran

Background and objective: Severe headaches are one of the most important problems in therapeutic process of chronic hepatitis $C$ with ribavirin and interferon alpha-2b. There are few previous reports of migraine headaches triggered by ribavirin in combination with interferon alpha-2b for chronic hepatitis $C$. The aim was to evaluate new emerging headaches in patients with chronic hepatitis $C$ under ribavirin plus interferon alpha-2b combination therapy.

Methods and materials: We enrolled 54 (38 male and 16 female) patients with chronic hepatitis $\mathrm{C}$ and applied them with a headache diagnosis and status questionnaire according to IHS 2004 headaches diagnostic criteria. All patients were under combination therapy with ribavirin and interferon alpha- $2 \mathrm{~b}$ at least for 6 months.

Results: A total of 18 (12 male, 6 female) patients had complaints from severe headaches. Six migraine with aura-type, one migraine without aura-type and two tension type headache were seen with less than $1 \mathrm{yr}$ of duration, two patients were seen with tension-type headache with duration of $1-5 \mathrm{yr}$, and one female patient had chronic migraine with aura headaches.

Conclusions: Our results showed increased rate of even migraine-type headaches with ribavirin plus interferon alpha- $2 b$ treatment of chronic hepatitis $C$ patients, corroborating previous studies. However, in our study the association of headaches with any of drugs is not clear. More clinical and experimental studies are to be needed to more clarify the association and possible underlying mechanisms.

\section{P 098 \\ Challenging anaesthesia for intraoperative high-field 3-T MRI in a neurosurgical context}

M. A. C. Meyer, Evans, Docquier, Van Boven,

Duprez, Raftopoulos

Université Catholique de Louvain, St-Luc Hospital, Brussels, Belgium

Background and objective: An intraoperative high-field 3-T MRI is a challenging environment for anaesthesiologists. To ensure safe management in this environment, our team had to adapt its techniques. We report our experience of anaesthetic management of the first 21 patients in the presence of a very strong magnetic field from a 3-T MRI.

Methods and materials: The new MRI complex consists of two completely independent areas: an operating theatre and an MRI suite. They are built very close to each other, but separated from each other by an ante-room. This new concept of MRI suite allows the anaesthetist and surgeon to work in a familiar operating environment, as well as allowing the radiologist to use the MRI machine for normal diagnostic procedures when not used intraoperatively. The patient is carefully and safely prepared and electrically isolated to allow his transfer into the MRI suite. A laminar pulsed-air cleaning system provides the aseptic requirements. The intraoperative transfer into the MRI suite is provided by a fully motorized operating table. Continuous MRI-compatible trolley monitoring is wheeled into the MRI suite. A special breathing circuit is used during the transfer to maintain inhalational anaesthesia.

Results: In all, 26 iMRI examinations were carried out. Of these, three were immediately pre-surgical, nine intra-surgical and 14 immediately post-surgical. Five patients had two iMRI procedures during their operations and three patients had further tumour resection immediately post iMRI. There were no iMRI accidents, although two cases of second-degree skin burn were observed, without any after-effect.

Discussion and conclusion: Rigorous check lists and safety rules were established in order to avoid making serious mistakes in the environment of a high-field 3-T MRI. The team was trained and experienced. Given these conditions, 3-T iMRI anaesthesia appears safe and reliable.

Furthermore, the success of the iMRI suite is the result of multidisciplinary teamwork.

\section{P 099}

\section{Awake craniotomy surgery in the United Kingdom - a survey of current anaesthetic practice}

A. Golding, C. Weidmann

Wessex Neurological Centre, Department of Southampton General Hospital, Southampton, UK

Background and objective: Demand from Neurosurgeons to provide services for awake neurosurgery is increasing. Awake surgery allows cortical mapping and maximal tumour resection, whilst preserving vital functions including speech and motor control. As more anaesthetists are likely to be called upon to provide these services we decided to undertake a national survey to gain an insight into current in practice.

Methods and materials: A questionnaire sent to all centres in the UK performing awake neurosurgery.

Results: A total of 19 centres were identified, we received responses from $14(74 \%)$ of these, comprising of 41 Consultant Anaesthetists. The majority (88\%) of Anaesthetists are performing 10 or less cases per year, with the most common indication being tumour resection (47\%). The anaesthetic sequence used could be broadly divided into a GA technique with awakening to allow 
functional testing, employed by $79.6 \%$ of respondents whist over $20 \%$ used a sedation only technique. For both techniques the majority of anaesthetists (79\%) reported using a combination of an anaesthetic agent and an opioid ( $80 \%$ choosing intravenous propofol and $88 \%$ using remifentanil as their opioid of choice). Adverse events reported included seizure activity (65\%) and although there were no reports of emergency intubation up to $63 \%$ of patients required airway support at some point. Postoperative care was split 50:50 between routine ward and HDU. Discharge from hospital was possible within $72 \mathrm{~h}$ in $46 \%$ of patients. Conclusions: Use of sedation based techniques are becoming more widespread as the choice of drugs and delivery systems improves. Despite some variations in practice across the UK, adverse event reporting was consistently low, indicating the safe nature of the procedure. Discharge times at present are mainly greater than $72 \mathrm{~h}$; we feel that this is likely to decrease with increasing familiarity with the procedures and the more frequent use of the newer short acting anaesthetic agents.

\section{P 100}

\section{Paediatric Neuroanaesthesia database creation: what information should we collect?}

T. Valois

Montreal Children's Hospital, Department of Anesthesia,

Montreal, Canada

Background and objective: With the advent of computers that possess improved capacity, databases have increased in popularity during the last $15 \mathrm{yr}$. Intraoperative computerized record keeping systems, has as well augmented our access to anaesthetic information in real time. However, measurable anaesthetic outcomes are difficult to define, despite improvements in data availability.

We would like to describe the creation process of a paediatric anaesthesia neurodatabase, data collection process and current uses in research and teaching.

Methods and materials: In 2004 a neurodatabase was created using Microsoft Access program, the design was developed by a paediatric anaesthetist with a special interest in neuroanaesthesia, the main goal was to collect intraoperative and postoperative data. Copy of anaesthetic record, as well as a form is provided by anaesthetists and the information is typed in the database. To date there are 300 patients.

Discussion: The database has been used for quality assurance purposes, retrospective reviews involving trainees and development of practice guidelines. The different difficulties encountered, process for defining anaesthetic outcomes and database maintenance are discussed.

\section{P 101}

\section{Serum myoglobin level during elective craniotomy}

A. Sekulic, M. Medjugorac, M. S. Miklandra, R. J Radonic, M. T. Murselovic

University Clinical Hospital Zagreb, Department of Division of Neuroanesthesia, Zagreb, Croatia

Background and objective: Elevated serum levels of myoglobin $(\mathrm{Mb})$ sometimes are clinically irrelevant, but possible toxic effects include renal failure, hyperkalemia, respiratory distress syndrome and death. A recent study showed elevated serum levels of $\mathrm{Mb}$ after elective neurosurgery. An operative procedure lasting more than $3 \mathrm{~h}$ was the only factor significantly correlated with peak $\mathrm{Mb}$ concentration [1]. The aim of our study was to detect possible earlier rises in serum levels of $\mathrm{Mb}$ during elective neurosurgery. Methods and materials: We tested serum $\mathrm{Mb}$ in fifteen neurosurgical patients, ASA I-III, age $56 \pm 15 \mathrm{yr}$ (mean \pm SD), who underwent elective craniotomy. Exclusion criteria were: hypothyreosis, alcohol abuse, use of statins, heart or skeletal disease, seizures and difficult endotracheal intubation. In a group of eleven patients, serum Mb levels were measured twice: $5 \mathrm{~min}$ before the induction of anaesthesia and $2 \mathrm{~h}$ after the first blood sample was drawned. Induction of anaesthesia was done by thiopental $5 \mathrm{mg} \mathrm{kg}^{-1}$, fentanyl $1.5 \mu \mathrm{g} \mathrm{kg}^{-1}$ and pancuronuim $0.1 \mathrm{mg} \mathrm{kg}^{-1}$. Maintenance of anaesthesia was performed with mixture of $33 \%$ oxygen with $67 \%$ nitrous oxide and continuous infusion of thiopental $5-8 \mathrm{mg} \mathrm{kg}^{-1} \mathrm{~h}^{-1}$ and fentanyl $1 \mu \mathrm{g} \mathrm{kg}^{-1} \mathrm{~h}^{-1}$. Serum Mb was measured by immunonephelometry. Statistical analysis was done by paired $t$-test for small dependent samples. $P$ value $<0.05$ was considered significant.

Results: There was a statistically significant difference in a comparison of serum myoglobin level before induction of anaesthesia $47.49 \pm 20.39 \mu \mathrm{gL}^{-1} \quad($ mean \pm SD) and $2 \mathrm{~h}$ after induction of anaesthesia $75.50 \pm 29.17 \mu \mathrm{gL}^{-1} ; \quad t=3.24$, $P=0.0088$.

Conclusion: The rise of serum myoglobin level was observed $2 \mathrm{~h}$ after induction of general anaesthesia for elective craniotomy.

\section{Reference}

1. Poli D, Gemma M, Cozzi S, Lugani D, Germagnoli L, Beretta L. Eur J Anaesthesiol 2007; 24(6): 551-555.

\section{P 102}

\section{Analgosedation and local anaesthesia for stereotactic biopsies of intracranial massess}

M. Symonides, P. Nauman, M. Lipiec, P. Sowinski, M. Chruscikowski, W. Bonicki

Institute of Oncology, Department of Anaesthesiology and Intensive Care, Warszawa, Poland

Background and objective: Stereotactic biopsies of intracranial tumours are performed in order to obtain correct diagnosis. We have adapted a regimen of sedation and local anaesthesia for these procedures and present an extended case report of 10 cases.

Methods and materials: Ten ASA III patients (6 males, 4 females), mean age $52 \mathrm{yr}$ (range 22-79), undergoing stereotactic biopsy of intracranial mass (mean duration $96 \mathrm{~min}$; range: 72-145 min); premedication: diazepam $5 \mathrm{mg}$ p.o., paracetamol $1 \mathrm{~g}$ p.o.; i.v. sedation: induction: dexamethasone $16 \mathrm{mg}$ i.v., midazolam: $1-2 \mathrm{mg}$ i.v., atropine $0.5 \mathrm{mg}$ i.v., propofol: $0.3-0.5 \mathrm{mg} \mathrm{kg}^{-1}$; remifentanil $0.25 \mu \mathrm{g} \mathrm{kg}^{-1}$; maintenance: remifentanil $0.07-0.2 \mu \mathrm{g} \mathrm{kg}^{-1} \mathrm{~min}^{-1}$; propofol $150-300 \mathrm{mg} \mathrm{h}^{-1}$. After induction a naso-pharyngeal tube was placed and oxygen insuflated via catheter while local anaesthesia of the scalp for pin fixation and biopsy was performed with $0.5 \%$ marcaine with adrenalin. Monitoring included $\mathrm{ABP}, \mathrm{HR}, \mathrm{S}_{\mathrm{P}} \mathrm{O}_{2}, \mathrm{BPM}$ and level of sedation.

Results: After induction patients slept for 8-10 min (Ramsay V$\mathrm{VI})$, facilitating NPT placement and local anaesthesia/pin placement. We observed no loss of spontaneous ventilation in $9 / 10$ cases; 1 patient developed apnoea and demanded assisted ventilation for $3 \mathrm{~min}$. A $10-15 \%$ transient increase in BP and HR was observed during the marcaine injection and no further reaction to frame fixation. Throughout the procedure, the patients were haemodynamically stable with $\mathrm{S}_{\mathrm{p}} \mathrm{O}_{2}$ within the $95-100 \%$ range; Ramsay score during the procedure was maintained at IIIIV. After the removal of the frame remifentanil and propofol were stopped and the patients were transferred to the recovery room. Oral paracetamol was maintained on day 1-2 after the biopsy and assured a VAS score of 0-2; three patients with VAS 2 were additionally given single doses of metamizole $2.5 \mathrm{~g}$ i.v. Recovery was uneventful. 
Conclusions: Stereotactic biopsies of brain masses may be safely conducted under sedation and local anaesthesia instead of general anaesthesia.

\section{P 103}

\section{Sufentanil for extensive neurosurgical procedures an extended case report of 63 cases}

M. Symonides, R. Krajewski, A. Ozieblo,

M. Chruscikowski, M. Misiak, P. Sowinski, P. Nauman,

W. Bonicki

Institute of Oncology, Department of Anaesthesiology and Intensive Care, Warsaw, Poland

Background and objective: Sufentanil has caused a number of controversies in neuroanaesthesia as it was said to be associated with epileptic activity, however recent studies report it's successful use in neurosurgery [1].

Methods and materials: Between October 2005 and November 2006 sufentanil was used for 63 extensive neurosurgical procedures of over $6 \mathrm{~h}$ : 34 supratentorial tumours (t.), 15 infratentorial t. and 14 cranial base t. Anaesthetic management included: induction: sufentanil $0.5-1.0 \mu \mathrm{g} \mathrm{kg}^{-1}$; midazolam $2 \mathrm{mg}$, thiopenthone $4-5 \mathrm{mg} \mathrm{kg}^{-1}$ and rocuronium $0.6 \mathrm{mg} \mathrm{kg}^{-1}$; maintenance: sevoflurane $2.0-3.5 \mathrm{vol} \%$ in air/oxygen, rocuronium inf. $10 \mu \mathrm{g} \mathrm{kg}^{-1} \mathrm{~min}^{-1}$ and sufentanil infusion $10-20 \mu \mathrm{g} \mathrm{h}^{-1}$. Rocuronium was stopped and sufentanil decreased to $5 \mu \mathrm{gh}^{-1}$ approximtely $40 \mathrm{~min}$ before the end of case. Evaluation: haemodynamic stability, emergence and any possible seizure activity

Results: We observed no significant changes in either BP and HR throughout the procedures. In case of the 34/34 supratentorial craniotomies emergence was fast under $7 \mathrm{~min}$ after sevoflurane discont. facilitating extubation; 4/15 infratentorial t. patients were electively transferred to the ICU for assisted ventilation and controlled emergence (procedures over $8 \mathrm{~h}$ ). Overall, in the 49 craniotomy patients there were 12 cases of BP increases on emergence, demanding administration of antihyper-tensives. In all, 5/14 cranial base patients were electively ventilated mechanically to assure slow, controlled emergence (procedures lasting over $8 \mathrm{~h}$ ). In 9/14 cases emergence was approximately $6-8 \mathrm{~min}$, facilitating extubation.

We observed no case of seizure activity.

Conclusion: Sufentanil appears safe in extensive neurosurgica procedures facilitating adequate emergence and uncomplicated tracheal extubation.

\section{Reference}

1. Bilotta F, Caramia R, Paoloni FP et al. Eur J Anesthesiol 2006; 23: $1-6$.

\section{P 104}

\section{Anaesthesia complications in neonates with neural tube defect}

M. D. Hasani

University Clinical Center of Kosova, Department of Anesthesiology, Pristina, Yugoslavia

Background and objective: Neural tube defect (myelodysplasia) is an abnormality in fusion of the embryologic neural groove during the first month of gestation. Failure of neural tube closure results in a sac-like herniation of the meninges (meningocele), or a herniation of neural elements (myelomeningocele). Repair of these defects is a surgical emergency and neonates should be operated on in the first $24 \mathrm{~h}$ of life for closure of the defect. The very young age of population and the nature of surgery lead to high rate of complication. The aim of this study was to evaluate the anaesthesia complications and our experience in this field.

Methods and materials: We included 156 preterm and term neonates, 76 males and 80 females, who underwent surgical repair of these defects during the first 7 days of their life. Anaesthesia was maintained with inhalation of halothane or sevoflurane and fentanyl or intravenous ketamine and fentanyl. All the patients were bringing in prone position after intubation. Complications during anaesthesia and after extubation were registered. We also recorded time of operation, recovery time, level of defect and association with other anomalies.

Results: In 6 (3.9\%) patients with cervical meningocele, difficult intubation were recorded (Mallampati grade III). In 24 (15.4\%) neonates, bleeding exceeding more than $20 \%$ of the body mass and blood transfusion was performed. Intense coughing and laryngospasm after extubation was recorded in 10 (6.4\%) patients. Delayed awaking from anaesthesia result in 19 $(12.2 \%)$ neonates in which the meningocele associated with hydrocephalus. Only two neonates required mechanical ventilation after anaesthesia, with duration no more than $24 \mathrm{~h}$.

Conclusion: Neonatal anaesthetic management in neural tube defects requires an understanding of the pharmacophysiologic limitations of the neonate as well as the pathophysiology of coexisting surgical disease. 